\title{
S-duality wall of SQCD from Toda braiding
}

\section{B. Le Floch}

Princeton Center for Theoretical Science, Princeton University, Princeton, NJ 08544, U.S.A.

Philippe Meyer Institute, Physics Department, École Normale Supérieure, PSL Research University, 24 rue Lhomond, F-75231 Paris Cedex 05, France

E-mail: bruno.le.floch@ens.fr

ABSTRACT: Exact field theory dualities can be implemented by duality domain walls such that passing any operator through the interface maps it to the dual operator. This paper describes the S-duality wall of four-dimensional $\mathcal{N}=2 \mathrm{SU}(N)$ SQCD with $2 N$ hypermultiplets in terms of fields on the defect, namely three-dimensional $\mathcal{N}=2$ SQCD with gauge group $\mathrm{U}(N-1)$ and $2 N$ flavours, with a monopole superpotential. The theory is self-dual under a duality found by Benini, Benvenuti and Pasquetti, in the same way that $T[\operatorname{SU}(N)]$ (the S-duality wall of $\mathcal{N}=4$ super Yang-Mills) is self-mirror. The domain-wall theory can also be realized as a limit of a $\operatorname{USp}(2 N-2)$ gauge theory; it reduces to known results for $N=2$. The theory is found through the AGT correspondence by determining the braiding kernel of two semi-degenerate vertex operators in Toda CFT.

Keywords: Supersymmetry and Duality, Conformal and W Symmetry, Duality in Gauge Field Theories, Supersymmetric Gauge Theory

ArXiv EPrint: 1512.09128 


\section{Contents}

1 Introduction 1

2 AGT relation $\quad 4$

2.1 Localization 4

2.2 Toda CFT 6

2.3 Special functions 8

3 Braiding matrices $\quad 9$

$\begin{array}{lr}3.1 \text { Braiding a fundamental degenerate } & 10\end{array}$

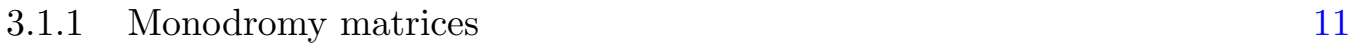

$\begin{array}{lll}3.1 .2 & \text { Explicit conformal blocks (proof) } & 12\end{array}$

$\begin{array}{ll}3.1 .3 \text { Braiding matrices } & 14\end{array}$

$\begin{array}{ll}3.2 & \text { Braiding a symmetric degenerate } \\ \end{array}$

4 Braiding kernel $\quad 19$

$\begin{array}{ll}4.1 & \text { Main formula } \\ & 19\end{array}$

$\begin{array}{lll}4.2 & \text { Reduction to fully degenerate } & 21\end{array}$

$\begin{array}{lll}\text { 4.3 Shift relation from pentagon identity } & 23\end{array}$

5 Domain wall and its symmetries $\quad 26$

$\begin{array}{ll}5.1 \text { Continuous flavour symmetries } & 26\end{array}$

$\begin{array}{ll}5.2 \text { Partition function } & 29\end{array}$

$\begin{array}{lll}5.2 .1 & \mathrm{U}(N-1) \text { description } & 29\end{array}$

5.2.2 USp $(2 N-2)$ description $\quad 31$

$\begin{array}{lll}5.3 & \text { Discrete symmetries and dualities } & 32\end{array}$

6 Conclusions $\quad 35$

\section{Introduction}

Extended operators, such as Wilson and 't Hooft loops [1, 2], surface operators [3-5], and domain walls [6-8] can serve as order parameters $[1,2,9]$ and help probe dualities of gauge theories. Exact all-scale dualities such as S-duality map all correlators of one theory to the dual theory through some dictionary that must be worked out on a case by case basis. For instance, S-duality of four-dimensional $\mathcal{N}=2$ supersymmetric gauge theories $[10,11]$ interchanges Wilson (electric) and 't Hooft (magnetic) loop operators [12-15], as befitting of this generalization of electric-magnetic duality.

Given an exact duality between theories $\mathcal{T}_{1}$ and $\mathcal{T}_{2}$, what happens if one dualizes one half-space, say $y \geq 0$ ? Start with $\mathcal{T}_{1}$ on all of space. After dualizing, the system should be described by $\mathcal{T}_{1}$ for $y<0$ and $\mathcal{T}_{2}$ for $y>0$, separated by a codimension 1 interface (wall) at 
$y=0$. Only a specific choice of interface can reproduce the physics of the original system. For instance, the position of the wall should not be observable so it must be topological away from operator insertions. When the interface passes through an operator inserted in the region described by theory $\mathcal{T}_{2}$, the operator should be replaced by its dual description in theory $\mathcal{T}_{1}$ to keep correlators fixed. Such duality walls [8] are thus a compact way to encode how dualities act on operators [16-22].

The duality wall can often be described by coupling bulk fields of theories $\mathcal{T}_{1}$ and $\mathcal{T}_{2}$ near the wall to additional degrees of freedom that live only on the wall. To determine what fields to put on the wall, one should decouple the bulk theories by making their couplings weak. This is impossible to do directly in the most common case of a weak-strong duality, as making the bulk coupling small on one side of the wall makes the other bulk theory strongly coupled. To avoid this we begin with a system described by theory $\mathcal{T}_{1}$ throughout space, with a Janus domain wall, namely a $y$-dependent coupling that varies sharply near $y=0$ from some value to another. Now dualize the $y>0$ side to get theories $\mathcal{T}_{1}$ and $\mathcal{T}_{2}$ for $y \gtrless 0$, separated by a duality wall as before. The couplings on the two sides can now be sent to zero independently to decouple bulk dynamics from the theory on the interface. If enough is known about Janus walls the codimension 1 theory can be deduced [8, 23-30].

For $4 \mathrm{~d} \mathcal{N}=4$ super Yang-Mills (SYM), $1 / 2$ BPS domain walls and boundary conditions were extensively studied in [6-8] (and 1/4 BPS ones in [31, 32]). S-duality of $\mathcal{N}=4$ SYM changes the gauge group $G$ to its Langlands dual ${ }^{L} G$, and the S-duality wall is described by gauging global symmetries $G \times{ }^{L} G$ of a $3 \mathrm{~d} \mathcal{N}=4$ theory $T[G]$ using the $3 \mathrm{~d}$ restrictions of the $4 \mathrm{~d}$ vector multiplets on both sides of the wall. The analysis in [8] largely relies on realizing $\mathcal{N}=4 \mathrm{SYM}$ as the world-sheet theory of $N$ D3 branes in the $G=\mathrm{SU}(N)$ case (other classical gauge groups are obtained using O3 planes), realizing boundary conditions by ending D3 branes on D5 or NS5 branes and noting that S-duality of $\mathcal{N}=4$ SYM descends from S-duality in IIB string theory. The $T[\mathrm{SU}(N)]$ theory is found to be given by the following brane configuration from which one reads a quiver gauge theory description:

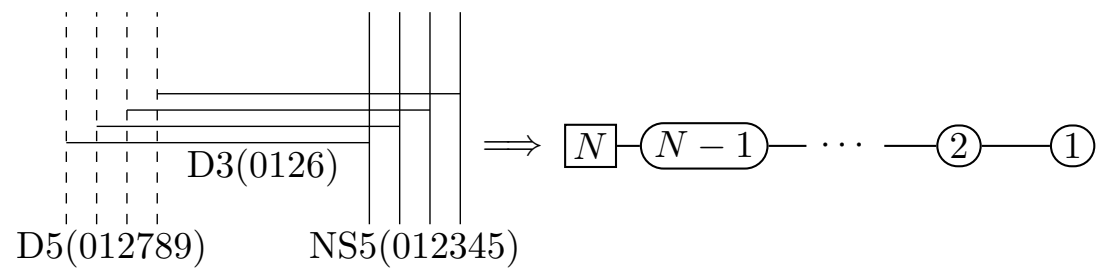

More precisely, $T[\mathrm{SU}(N)]$ is the infrared limit of the gauge theory with gauge group $\mathrm{U}(N-1) \times \mathrm{U}(N-2) \times \cdots \times \mathrm{U}(1)$ and hypermultiplets in the bifundamental representation of each pair $\mathrm{U}(k) \times \mathrm{U}(k-1)$, as well as $N$ hypermultiplets in the fundamental representation of $\mathrm{U}(N-1)$, which have an $\mathrm{SU}(N)$ flavour symmetry. Since IIB S-duality interchanges D5 and NS5 branes, leaving the brane diagram essentially invariant, $T[\mathrm{SU}(N)]$ is self-mirror so its $\mathrm{U}(1)^{N-1}$ topological symmetry enhances to another ${ }^{1} \mathrm{SU}(N)={ }^{L} \mathrm{SU}(N)$. However, this construction through IIB strings does not apply to S-duality of $4 \mathrm{~d} \mathcal{N}=2$ theories. $^{2}$

\footnotetext{
${ }^{1}$ Throughout we will ignore global aspects of flavour symmetries: here one $\mathrm{SU}(N)$ should be $\operatorname{PSU}(N)$.

${ }^{2}$ One exception is that the world-volume theory of fractional D3 branes at the center of an orbifold $\mathbb{R}^{4} / \mathbb{Z}_{k}$ is a $4 \mathrm{~d} \mathcal{N}=2$ necklace quiver. However, IIB string theory only provides $\mathrm{SL}(2, \mathbb{Z})$ S-dualities acting on all gauge couplings at once, while there exists a much richer set of dualities acting "locally" on the quiver.
} 
For the large class of $4 \mathrm{~d} \mathcal{N}=2 \mathrm{SU}(N)$ gauge theories obtained by twisted dimensional reduction of $6 \mathrm{~d}(2,0) A_{N-1}$ superconformal theory on a punctured Riemann surface $\Sigma$, the AGT correspondence provides another handle [33] (see also [34-36]). It relates observables of the $4 \mathrm{~d}$ theory on an ellipsoid $S_{b}^{4}$ to observables of the Toda CFT on $\Sigma$, a generalization of the Liouville CFT with a larger symmetry algebra $W_{N}$. In particular, an S-duality domain wall placed along the equator (or a parallel) $S_{b}^{3}$ of $S_{b}^{4}$ corresponds to a certain $W_{N}$ braiding transformation [15]. From the integral kernel that braids certain $W_{N}$ primary operators of the Toda CFT one can thus deduce the partition function of the S-duality domain wall, hence the $S_{b}^{3}$ partition function of its description as a $3 \mathrm{~d}$ gauge theory, as we explain in Section 2. One is then left with finding a $3 \mathrm{~d}$ gauge theory with the given $S_{b}^{3}$ partition function. This strategy was applied in [23] to the mass deformation of $4 \mathrm{~d} \mathcal{N}=4$ and in [37] to $4 \mathrm{~d} \mathcal{N}=2 \mathrm{SU}(2) \mathrm{SQCD}$ with 4 fundamental hypermultiplets.

While we concentrate on $4 \mathrm{~d} \mathcal{N}=2 \mathrm{SU}(N)$ SQCD with $2 N$ fundamental hypermultiplets, the $3 \mathrm{~d} \mathcal{N}=2$ description of the SQCD S-duality wall that we give momentarily also implements S-duality of any balanced $\mathrm{SU}(N)$ gauge node in a $4 \mathrm{~d} \mathcal{N}=2$ quiver (see the conclusion section). We have not worked out the case of $N_{f}<2 N$ hypermultiplets, but it should simply amount to making some hypermultiplets massive.

We determine the relevant braiding kernel (4.4) in Section 4 as a continuation of braiding matrices (3.42) worked out in Section 3 using 2d CFT pentagon relations. Comparing it to explicit expressions of $S_{b}^{3}$ partition functions from localization [38-40] we describe in Section 5 the S-duality wall of $4 \mathrm{~d} \mathcal{N}=2 \mathrm{SU}(N)$ SQCD as $3 \mathrm{~d} \mathcal{N}=2 \mathrm{U}(N-1)$ SQCD with a monopole superpotential on the wall, coupled to the $4 \mathrm{~d}$ theories on both sides of the wall. We then find evidence that the $3 \mathrm{~d}$ theory can also be obtained as a certain limit of an $\operatorname{USp}(2 N-2)$ theory, in which some symmetries are more manifest. Altogether,

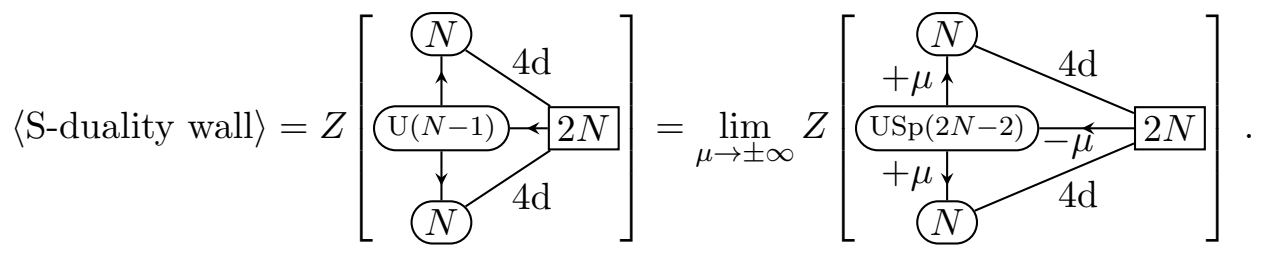

In these quiver, the upper and lower round nodes labelled $N$ denote $\mathrm{SU}(N)$ gauge groups of the $4 \mathrm{~d}$ theories on both sides of the wall, the diagonal edges stemming from them are $2 N$ hypermultiplets (again on both sides of the wall) that share a common $\mathrm{SU}(2 N)$ flavour symmetry across the wall. The rest of the quiver describes the $3 \mathrm{~d} \mathcal{N}=2$ theory on the wall: a $\mathrm{U}(N-1)$ or $\mathrm{USp}(2 N-2)$ vector multiplet coupled to $2 N$ fundamental and $N+N$ antifundamental chiral multiplets. The labels $\pm \mu$ will be explained shortly. Additionally, the $3 \mathrm{~d}$ and $4 \mathrm{~d}$ matter multiplets are coupled by a cubic superpotential $W$ along the defect: ${ }^{3}$

$$
W=\sum_{f=1}^{2 N} \sum_{s=1}^{N}\left(\left.\Phi_{f s}\right|_{3 \mathrm{~d}} \widetilde{q}_{s} q_{f}+\left.\Phi_{f s}^{\prime}\right|_{3 \mathrm{~d}} \widetilde{q}_{s}^{\prime} q_{f}\right)+ \begin{cases}V_{+}+V_{-} & \text {for the } \mathrm{U}(N-1) \text { theory } \\ Y & \text { for the } \mathrm{USp}(2 N-2) \text { theory. }\end{cases}
$$

\footnotetext{
${ }^{3}$ Version 1 of this paper was lacking $V_{+}+V_{-}$and $Y$ so the proposed $4 \mathrm{~d} / 3 \mathrm{~d}$ system had too many symmetries. Thanks to Sara Pasquetti for pointing this out. In fact, the corrected $3 \mathrm{~d}$ theory with $W=$ $V_{+}+V_{-}$is self-dual: see [41] for $N=2$ and [42] (after our version 1) for all $N$. This duality turns out to make the $\operatorname{USp}(2 N-2)$ theory unnecessary for our purposes, but we keep it as an interesting variant.
} 
Here, $\widetilde{q}_{s}$ and $\widetilde{q}_{s}$ denote the $2 N$ antifundamental chiral multiplets and $q_{f}$ the $2 N$ fundamental ones, while $\left.\Phi\right|_{3 \mathrm{~d}}$ and $\left.\Phi^{\prime}\right|_{3 \mathrm{~d}}$ are limits of $4 \mathrm{~d}$ hypermultiplets at the interface. Following standard notations, $V_{ \pm}$are monopole operators with $\mathrm{U}(1)_{T}$ charges \pm 1 in the $\mathrm{U}(N-1)$ theory, while for the $\operatorname{USp}(2 N-2)$ theory the monopole is denoted by $Y$ (see, e.g., [43]). Coefficients of the superpotential cannot be determined by our methods.

The rest of this paper is organized as follows. Section 2 explains the AGT correspondence relating the S-duality domain wall of $4 \mathrm{~d} \mathcal{N}=2 \mathrm{SU}(N)$ SQCD to a $W_{N}$ braiding kernel $^{4}$ and gives an introduction to the Toda CFT. Section 3 evaluates some braiding matrices (3.42) which are special cases of the braiding kernel when an operator is fully degenerate. Section 4 generalizes these discrete results to a continuous braiding kernel (4.4). It describes how this integral kernel reduces to Liouville CFT braiding kernels $[44,45]$ and proves that the kernel obeys a shift relation deduced from Moore-Seiberg pentagon identities [46]. A reader with no interest in Toda CFT can jump directly to section 5, where we extract the above gauge theory description of the S-duality wall from the braiding kernel and explain how continuous flavour symmetries and various $\mathbb{Z}_{2}$ symmetries (including selfdualities) match between the different descriptions. We conclude in section 6 with some remarks on class $\mathrm{S}$ quiver gauge theories.

\section{AGT relation}

This section describes supersymmetric localization for $4 \mathrm{~d} \mathcal{N}=2$ theories, then translates S-duality domain walls of $\mathrm{SU}(N)$ SQCD to the $2 \mathrm{~d}$ CFT language using the AGT correspondence.

\subsection{Localization}

For a given choice of supercharge $\mathcal{Q}$ under which a path integral is invariant, localization reduces the path integral to a simpler integral over $\mathcal{Q}$-invariant field configurations only. ${ }^{5}$ As an IR cutoff we place the theory on the ellipsoid $S_{b}^{4}$ given in coordinates as

$$
x_{0}^{2}+b^{-2}\left(x_{1}^{2}+x_{2}^{2}\right)+b^{2}\left(x_{3}^{2}+x_{4}^{2}\right)=1 .
$$

Preserving supersymmetry requires non-trivial background fields [35]. There exists another deformation of the sphere in which the parameter $b$ is complex rather than real [36]. Domain walls will be placed at constant $x_{0}$, such as the equator $S_{b}^{3}$ at $x_{0}=0$.

The partition function of $4 \mathrm{~d} \mathcal{N}=2$ theories on $S_{b}^{4}$ was computed [35, 47] using a supercharge $\mathcal{Q}$ whose square rotates the ellipsoid in the planes $\left(x_{1}, x_{2}\right)$ and $\left(x_{3}, x_{4}\right)$ and leave two poles $x_{0}= \pm 1$ fixed. In $\mathcal{Q}$-invariant configurations one vector multiplet scalar

\footnotetext{
${ }^{4}$ While the braiding kernel is only sensitive to the chiral symmetry algebra $W_{N}$ we will sometimes call it Toda CFT braiding kernel because Toda CFT is the prime example of $W_{N}$-symmetric theory.

${ }^{5}$ More precisely, localization relies on a choice of $\mathcal{Q}$-closed and $\mathcal{Q}$-exact (and positive) deformation term $\delta S$ for the action: the deformed path integral including a factor $\exp (-t \delta S)$ is then independent of $t \geq 0$. At large $t$ the saddle point approximation is exact. For an appropriate $\delta S$, saddle points are $\mathcal{Q}$-invariant configurations.
} 
takes a constant value i $a$ and other fields vanish, except at the North and South poles where there can be instantons and anti-instantons. The exact $S_{b}^{4}$ partition function is then

$$
Z_{S_{b}^{4}}=\int \mathrm{d} a e^{-S_{\mathrm{cl}}(a, x, \bar{x})} Z_{1-\text { loop }}(m, a) Z_{\text {inst }}(m, a, x) Z_{\text {anti-inst }}(m, a, \bar{x})
$$

where the real scalar $a$ runs over the gauge Lie algebra, $x$ stands for exponentiated gauge coupling constants and $m$ for the masses. The integrand consists of a classical contribution $\exp \left(-S_{\mathrm{cl}}\right)$ evaluated at the saddle point (away from the poles), a one-loop determinant due to fluctuations around the saddle point, and (anti)instanton contributions at the poles, which depend (anti)holomorphically on $x$. These instanton partition functions are power series that normally converge in a finite region near the weakly-coupled limit $x \rightarrow 0$. After reducing the integral to the Cartan algebra, combining the resulting Vandermonde determinant with $Z_{1 \text {-loop }}$, and factorizing the classical contribution into holomorphic functions of $x$ and $\bar{x}$, one gets (2.3) below. S-dual theories have equal ellipsoid partition functions, hence

$$
\begin{aligned}
Z_{S_{b}^{4}} & =\int \mathrm{d} a C(m, a) f(m, a, x) f(m, a, \bar{x}) \\
& =\int \mathrm{d} a_{D} C_{D}\left(m, a_{D}\right) f_{D}\left(m, a_{D}, x_{D}\right) f_{D}\left(m, a_{D}, \bar{x}_{D}\right) .
\end{aligned}
$$

While the global symmetries and masses $m$ of the two $4 \mathrm{~d}$ theories are shared up to permutations, their matter content and coupling constants may be very different. For a fixed $\bar{x}$ (and $\bar{x}_{D}$ ) the two integral representations express $Z$ in different bases of holomorphic functions $f$ and $f_{D}$, hence there should exist a change of basis $B\left(m, a, a^{\prime}\right)$, called S-duality kernel:

$$
f(m, a, x)=\int \mathrm{d} a^{\prime} B\left(m, a, a^{\prime}\right) f_{D}\left(m, a^{\prime}, x_{D}\right) .
$$

Let us repeat the localization computation, but with a Janus domain wall that interpolates between $x$ in the North half-ellipsoid and $x^{\prime}$ in the South half-ellipsoid. While the $\mathcal{Q}$-invariant field configurations are unaffected by the wall, the (anti)instanton contributions from the North and South poles are evaluated using $x$ and $\bar{x}^{\prime}$, respectively. The classical contribution is affected in a similar way [15, section 5], and one gets

$$
\langle\text { Janus wall }\rangle=\int \mathrm{d} a C(m, a) f(m, a, x) f\left(m, a, \bar{x}^{\prime}\right) .
$$

When the couplings are S-dual $\left(\bar{x}_{D}^{\prime}=\bar{x}\right)$, one can apply S-duality (2.5) to the South half-ellipsoid and obtain a duality wall:

$$
\langle\text { S-duality wall }\rangle=\int \mathrm{d} a \int \mathrm{d} a^{\prime} C(m, a) B\left(m, a, a^{\prime}\right) f(m, a, x) f_{D}\left(m, a^{\prime}, \bar{x}\right) .
$$

On the other hand, consider the $4 \mathrm{~d} \mathcal{N}=2$ theory on $S_{b}^{4}$ with coupling $x$ throughout the ellipsoid, coupled to $3 \mathrm{~d}$ fields on the equator. $\mathcal{Q}$-invariant configurations of such a $4 \mathrm{~d} / 3 \mathrm{~d}$ system have constant vector multiplet scalars $\mathrm{i} a$ and $\mathrm{i} a^{\prime}$ on the two halves of $S_{b}^{4}$, instantons at each pole, and possibly additional non-trivial field configurations on the $3 \mathrm{~d}$ equator. The contribution from $3 \mathrm{~d}$ fields is simply the $S^{3}$ partition function of the theory 
obtained by freezing the $4 \mathrm{~d}$ fields to their values (i $\left.a, \mathrm{i} a^{\prime}\right)$. The $4 \mathrm{~d}$ classical and instanton contributions combine as before into $f(m, a, x)$ and $f_{D}\left(m, a^{\prime}, \bar{x}\right)$ (provided an appropriate Chern-Simons term is included on the wall), and finally $4 \mathrm{~d}$ one-loop determinants on each half-ellipsoid are functions of $(m, a)$ and of $\left(m, a^{\prime}\right)$. Altogether, (2.7) can be reproduced provided one finds a $3 \mathrm{~d}$ theory whose ellipsoid partition function is essentially $B\left(m, a, a^{\prime}\right)$ :

$$
Z_{S_{b}^{3}}\left(m, a, a^{\prime}\right)=\frac{C(m, a) B\left(m, a, a^{\prime}\right)}{Z_{1 \text {-loop }}^{\text {half-ellipsoid }}(m, a) Z_{1 \text {-loop }}^{\text {half-ellipsoid }}\left(m, a^{\prime}\right)} .
$$

In particular, the $3 \mathrm{~d}$ partition function should depend on $4 \mathrm{~d}$ masses and vector multiplet scalars $\left(m, a, a^{\prime}\right)$ which lie in the Cartan algebras of the global symmetry group (shared by the two $4 \mathrm{~d}$ theories) and of both gauge groups. The $3 \mathrm{~d}$ theory must therefore contain fields charged under each of these symmetries. For instance, the domain wall theory $T[\mathrm{SU}(N)]$ of $4 \mathrm{~d} \mathcal{N}=4 \mathrm{SU}(N) \operatorname{SYM}(1.1)$ has a (non-manifest) global symmetry $\mathrm{U}(1) \times \mathrm{SU}(N)^{2}$.

We are interested in $4 \mathrm{~d} \mathcal{N}=2 \mathrm{SU}(N) \mathrm{SQCD}$ with $2 N$ flavours. Let $m_{f}(f=1, \ldots, 2 N)$ be the masses of these hypermultiplets. S-duality inverts the $\mathrm{U}(1)$ subgroup of the $\mathrm{U}(2 N)$ flavour symmetry: namely masses in the dual theory are $m_{f}-2 m$, where $m=\frac{1}{2 N} \sum_{f} m_{f}$. The $3 \mathrm{~d}$ gauge theory description, which we find in section 5 , must thus have the flavour symmetry $\mathrm{U}(1) \times \mathrm{SU}(2 N) \times \mathrm{SU}(N) \times \mathrm{SU}(N)$ (up to discrete factors). This symmetry is explicitly broken by $\left(m, a, a^{\prime}\right)$.

To find this $3 \mathrm{~d}$ gauge theory description we must evaluate the right-hand side of (2.8). The relevant structure constants $C(m, a)$ are known. The one-loop determinants of $4 \mathrm{~d}$ vector and hypermultiplets are only known on the full ellipsoid and not on a half-ellipsoid. We take as inspiration the analoguous situation in two dimensions: sphere one-loop determinants involve a combination $\Gamma(x) / \Gamma(1-x)$ while hemisphere ones involve $\Gamma(x)$ or $1 / \Gamma(1-x)$ depending on boundary conditions [48-50]. In four dimensions, we note that the one-loop determinant of a hypermultiplet of mass $m$ is ${ }^{6} \Gamma_{b}\left(\frac{b+b^{-1}}{2}+\mathrm{i} m\right) \Gamma_{b}\left(\frac{b+b^{-1}}{2}-\mathrm{i} m\right)$. It is thus natural to propose that the one-loop determinant on a hemisphere (or rather a half-ellipsoid) is a single one of these two $\Gamma_{b}$ functions. ${ }^{7}$ The situation is similar for vector multiplets. Even if the proposal turns out to be incorrect, our main statement identifying what $3 \mathrm{~d}$ theory to couple to the two $4 \mathrm{~d}$ theories will hold, since one-loop determinants depend on $a$ and $a^{\prime}$ separately. The most important ingredient in (2.8) is the S-duality kernel $B$, which mixes $a$ and $a^{\prime}$.

\subsection{Toda CFT}

To determine the kernel $B$ we will use the AGT correspondence [33], found by remarking that the various factorizations (2.3) and (2.4) of $Z$ into holomorphic factors are reminiscent of conformal block decompositions in 2d CFT. Observables of $4 \mathrm{~d} \mathcal{N}=2 \mathrm{SU}(N)$ gauge theories (of class S) on $S_{b}^{4}$ are equal to observables in the $A_{N-1}$ Toda CFT.

\footnotetext{
${ }^{6}$ See section 2.3 for properties of special functions.

${ }^{7}$ This was previously suggested for instance in [41, page 24] and [51, page 29].
} 
This generalization of the Liouville CFT (the case $N=2$ ) has a symmetry algebra $W_{N}$ (for $N=2$, the Virasoro algebra). We use standard notations. ${ }^{8}$ The vertex operators $\widehat{V}_{\alpha}$, whose momentum $\alpha$ depends on $N-1$ parameters, are $W_{N}$ primaries of dimension $\Delta(\alpha)=\frac{1}{2}\langle\alpha, 2 Q-\alpha\rangle$. They are invariant under Weyl transformations, which permute their components $\left\langle\alpha-Q, h_{i}\right\rangle .{ }^{9}$ Among these primary operators we call the oneparameter class of momenta $\alpha=\kappa h_{1}$ semi-degenerate, or simple, ${ }^{10}$ and the discrete set $\alpha=-K b h_{1}$ (for integer $K \geq 0$ ) fully degenerate. ${ }^{11}$

The $S_{b}^{4}$ partition function of $4 \mathrm{~d} \mathcal{N}=2 \mathrm{SQCD}$ is then equal to a sphere correlator of two generic and two simple vertex operators:

$$
Z_{S_{b}^{4}}(\mathrm{SQCD})=|x|^{2 \gamma_{0}}|1-x|^{2 \gamma_{1}}\left\langle\widehat{V}_{\alpha_{3}}(\infty) \widehat{V}_{\kappa_{4} h_{1}}(1) \widehat{V}_{\kappa_{2} h_{1}}(x, \bar{x}) \widehat{V}_{\alpha_{1}}(0)\right\rangle
$$

The cross-ratio $x$ of their positions is the exponentiated gauge coupling, mapped to $1 / x$ by S-duality. The unimportant exponents $\gamma_{i}$ can be fixed by matching Toda CFT and gauge theory asymptotics as $x \rightarrow 0, \infty$. The momenta $\alpha_{1}$ and $\kappa_{2} h_{1}$ encode $N$ hypermultiplet masses and $\alpha_{3}$ and $\kappa_{4} h_{1}$ the other $N$. The Toda CFT correlator thus does not make all gauge theory symmetries explicit, which leads to various sign asymmetries. Momenta are

$$
\begin{array}{ll}
\alpha_{1}=Q+\sum_{j=1}^{N}\left(\mathrm{i} m_{j} h_{j}\right) & \kappa_{2}=N\left(\frac{b+b^{-1}}{2}-\frac{1}{N} \sum_{j=1}^{N} \mathrm{i} m_{j}\right) \\
\alpha_{3}=Q-\sum_{j=1}^{N}\left(\mathrm{i} m_{j+N} h_{j}\right) & \kappa_{4}=N\left(\frac{b+b^{-1}}{2}+\frac{1}{N} \sum_{j=1}^{N} \mathrm{i} m_{j+N}\right) .
\end{array}
$$

From the Toda CFT point of view, the two S-dual decompositions (2.3) and (2.4) of the partition function are conformal block decompositions obtained by taking the operator product expansion (OPE) of $\widehat{V}_{\kappa_{2} h_{1}}(x, \bar{x})$ with either $\widehat{V}_{\alpha_{1}}(0)$ ("s-channel decomposition") or with $\widehat{V}_{\alpha_{3}}(\infty)$ ("u-channel decomposition"). Considering the s-channel for definiteness, the integration variable $a$ parametrizes the primary operator resulting from the OPE of $\widehat{V}_{\alpha_{1}}$ with $\widehat{V}_{\kappa_{2} h_{1}}$. The one-loop contributions $C(m, a)$ are structure constants of the Toda CFT,

\footnotetext{
${ }^{8}$ Let $h_{1}, \ldots, h_{N}$ denote the weights of the fundamental representation of $A_{N-1}$, which sum to 0 . These form an overcomplete basis of the Cartan algebra $\mathfrak{h}$, identified to $\mathfrak{h}^{*}$ by the Killing form defined by $\left\langle h_{i}, h_{j}\right\rangle=$ $\delta_{i j}-1 / N$. The highest weight of any representation of $A_{N-1}$ can be written $\Omega=n_{1} h_{1}+\cdots+n_{N} h_{N}$ with integers $n_{1} \geq \cdots \geq n_{N}=0$. We also let $\rho=\frac{1}{2} \sum_{i<j}^{N}\left(h_{i}-h_{j}\right)=\sum_{i=1}^{N} \frac{N+1-2 i}{2} h_{i}$ be the half-sum of positive roots and $Q=\left(b+b^{-1}\right) \rho$.

${ }^{9}$ Momenta are elements of $\mathfrak{h}$. Normalizability requires $\alpha-Q$ to be an imaginary element of $\mathfrak{h}$. The dimension of $V_{\alpha}$ and other quantum numbers are invariant under Weyl transformations, and it turns out that the Toda CFT has a single operator with a given set of quantum numbers, hence $V_{\alpha}$ itself is invariant up to a scalar. We choose a normalization $\widehat{V}_{\alpha}$ invariant under Weyl transformations; the normalization also avoids annoying constants in the AGT relation, and it does not affect conformal blocks nor braiding.

${ }^{10}$ Many authors consider semi-degenerate momenta of the form $\alpha=-\lambda h_{N}$. This choice is mapped to ours by a Weyl reflection: $-\lambda h_{N} \rightarrow\left(N\left(b+b^{-1}\right)-\lambda\right) h_{1}$.

${ }^{11}$ More generally, $\alpha=-b \Omega_{1}-b^{-1} \Omega_{2}$, where $\Omega_{1}$ and $\Omega_{2}$ are highest weights of two representations of $A_{N-1}$, are fully degenerate and form a discrete set. We will always take $\Omega_{2}=0$ and $\Omega_{1}=K h_{1}$, highest weight of the $K$-th symmetric representation of $A_{N-1}$. Both semi-degenerate and fully degenerate primary operators are interesting because there are null-vectors among their $W_{N}$ descendants.
} 
and the (anti)instanton partition functions $f(m, a, x)$ and $f(m, a, \bar{x})$ are (anti)holomorphic s-channel conformal blocks.

Four-point function of $W_{N}$ primary operators do not decompose so simply into (anti)holomorphic conformal blocks in general. Inserting a complete set of states (both primaries and their descendants) in a generic four-point function $\left\langle V_{4} V_{3} V_{2} V_{1}\right\rangle$ gives schematically $\int \mathrm{d} \alpha \sum_{I, \bar{I}}\left\langle V_{4} V_{3}\left(W_{-I} \bar{W}_{-\bar{I}} V_{\alpha}\right)\right\rangle\left\langle\left(W_{-I} \bar{W}_{-\bar{I}} V_{2 Q-\alpha}\right) V_{2} V_{1}\right\rangle$ where $W_{-I}$ and $\bar{W}_{-\bar{I}}$ are an orthonormal basis of the left/right-moving $W_{N}$ algebras and we used $\left\langle V_{\alpha} V_{2 Q-\alpha}\right\rangle=1$. When each three-point function feature a semi-degenerate vertex operator, its null-vectors can be used to convert the action of $W$ and $\bar{W}$ into that of Virasoro generators only, which are known to act as differential operators. For the four-point function (2.9) of interest the s-channel and u-channel have this property (not the t-channel), which guarantees that the contributions from descendants of a given primary operator factorize into conformal blocks.

The s-channel and u-channel decompositions converge in different regions $|x| \lessgtr 1$. In both cases, every conformal block is a fractional power of $x$ multiplied by a power series which converges in the unit disk. They can in fact be analytically continued to the whole plane minus branch cuts joining 0,1 , and $\infty$. A convenient choice will be to cut along $[0, \infty)$, in other words normalize conformal blocks so that their leading term is a fractional power of $(-x)$. We define the braiding kernel as the integral kernel expressing s-channel blocks in terms of u-channel ones after analytic continuation. This is the Toda CFT translation of the S-duality kernel defined in (2.5). For comparison we write formulas next to each other:

$$
\begin{aligned}
& f(m, a, x)=\int \mathrm{d} a^{\prime} B\left(m, a, a^{\prime}\right) f_{D}\left(m, a^{\prime}, x_{D}\right)
\end{aligned}
$$

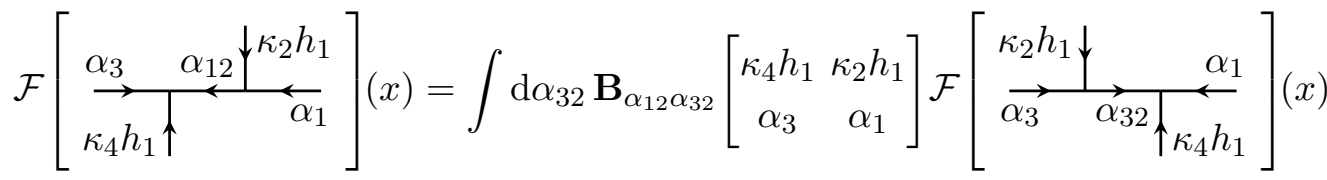

The $\alpha_{32}$ integral runs over imaginary values for $\alpha_{32}-Q$. The blocks $\mathcal{F}(x)$ and $f(x)$ differ by the same factor $(-x)^{\gamma_{0}}(1-x)^{\gamma_{1}}$ in both channels, hence $B\left(m, a, a^{\prime}\right)=\mathbf{B}_{\alpha_{12} \alpha_{32}}$.

In the normalization where the leading term of $f(x)$ is a power of $x$, the S-duality kernel is changed by a phase due to altered branch cuts. Using $x^{\lambda}=e^{\epsilon i \pi \lambda}(-x)^{\lambda}$ with $\epsilon=\operatorname{sign} \operatorname{Im} x$, and given the semi-classical limits $f(m, a, x) \sim x^{\frac{1}{2} \sum_{j} a_{j}^{2}}$ and $f(m, a, x) \sim x^{-\frac{1}{2} \sum_{j} a_{j}^{\prime 2}}$ as $x \rightarrow 0, \infty$, the S-duality kernel in this normalization is

$$
B^{\epsilon}\left(m, a, a^{\prime}\right)=e^{\epsilon \mathrm{i} \pi\left[\frac{1}{2} \sum_{j} a_{j}^{2}+\frac{1}{2} \sum_{j} a_{j}^{\prime 2}\right]} B\left(m, a, a^{\prime}\right) .
$$

We will interpret these phases as a Chern-Simons term on the wall. The braiding kernel receives similar phases.

Our goal is to find the kernel $\mathbf{B}_{\alpha_{12} \alpha_{32}}$. We determine it in section 3 when $\kappa_{2} h_{1}$ is fully degenerate, namely $\kappa_{2}=-K b$ with $K \geq 0$ an integer, then generalize to all $\kappa_{2}$ in section 4 .

\subsection{Special functions}

Besides the Barnes double-Gamma function $\Gamma_{b}=\Gamma_{1 / b}$, normalized by $\Gamma_{b}\left(\frac{b+b^{-1}}{2}\right)=1$, we need the double-Sine function $S_{b}(x)=S_{1 / b}(x)=\Gamma_{b}(x) / \Gamma_{b}\left(b+b^{-1}-x\right)=1 / S_{b}\left(b+b^{-1}-x\right)$, 


\begin{tabular}{|cccc|}
\hline & $-b \mathbb{Z}_{\geq 0}-\frac{1}{b} \mathbb{Z}_{\geq 0}$ & $b \mathbb{Z}_{\geq 1}+\frac{1}{b} \mathbb{Z}_{\geq 1}$ & Shift relation \\
\hline$\Gamma_{b}$ & poles & finite & $\Gamma_{b}(x+b) / \Gamma_{b}(x)=\sqrt{2 \pi} b^{x b-1 / 2} / \Gamma(x b)$ \\
$S_{b}$ & poles & zeros & $S_{b}(x+b) / S_{b}(x)=2 \sin (\pi b x)$ \\
$\Upsilon_{b}$ & zeros & zeros & $\Upsilon_{b}(x+b) / \Upsilon_{b}(x)=b^{1-2 b x} \gamma(b x)$ \\
\hline
\end{tabular}

Table 1. Properties of Barnes functions.

and the Upsilon function $\Upsilon_{b}(x)=\Upsilon_{1 / b}(x)=1 /\left(\Gamma_{b}(x) \Gamma_{b}\left(b+b^{-1}-x\right)\right)=\Upsilon_{b}\left(b+b^{-1}-x\right)$. All are meromorphic in $x$. Their zeros and poles are given in table 1 together with their shift relations expressed using $\gamma(y)=\Gamma(y) / \Gamma(1-y)$ and the Euler identity $\Gamma(y) \Gamma(1-y)=$ $\pi / \sin (\pi y)$. In addition, $S_{b}(b)=b$.

\section{Braiding matrices}

We determine here the braiding of Toda $\mathrm{CFT}^{12}$ four-point conformal blocks involving a semi-degenerate operator and a fully degenerate one labelled by the $K$-th symmetric representation $\mathcal{R}\left(K h_{1}\right)$ of $A_{N-1}$. Fusion rules of the fully degenerate operator ${ }^{13}$ reduce the four-point function to a finite sum (rather than an integral) of holomorphic times antiholomorphic conformal blocks. There are $\operatorname{dim} \mathcal{R}\left(K h_{1}\right)=\left(\begin{array}{c}N+K-1 \\ K\end{array}\right)$ terms. Braiding is thus given by square matrices of this size.

We begin in section 3.1 with the fundamental representation, so $\alpha=-b h_{1}$. In that case, conformal blocks are known to be hypergeometric functions. This was initially obtained by solving the null-vector differential equations for $N=3$ and writing the natural generalization of these results [53]. Fuchsian techniques provide a proof for all $N$ when $c=N-1$, namely $b^{2}=-1$ [52, Theorem 4.1] (which appeared after version 1 of this paper). We outline a mild variation of their proof that generalizes the result to all $b$, under the assumption that well-known fusion rules are correct. Then, as a preparation for more difficult calculations, we review the braiding matrix for these hypergeometric functions that forms the foundation of our new results.

We do not discuss here the case $\alpha=-b h_{1}-\cdots-b h_{K}$ of antisymmetric representations, treated in collaboration with Gomis in [54]. We conjectured there that conformal blocks are vortex partition functions of $2 \mathrm{~d} \mathcal{N}=(2,2) \mathrm{SQCD}$. In appendix A.3 of that paper we computed the braiding matrix of the gauge theory result, then showed using shift relations that it is the correct braiding matrix for conformal blocks. This method fixes braiding matrices hence monodromies, but one would need to adapt the proof from the fundamental case to show that the conformal blocks conjectured from gauge theory are indeed correct.

In section 3.2 we turn to the $K$-th symmetric representation, so $\alpha=-K b h_{1}$, starting from a conjecture for conformal blocks as gauge theory vortex partition functions [54].

\footnotetext{
${ }^{12}$ Most results apply to all CFTs with $W_{N}$ symmetry, but to be fully general would require various multiplicity indices: there may be several primary operators with the same momentum.

${ }^{13}$ Fusion rules are typically found using a Coulomb gas representation of Toda CFT correlators. They have only been proven rigorously in representation theory of $W_{N}$ algebras in the simplest case: the fundamental representation, for $b^{2}=-1$ (so as to have a free boson realization) [52].
} 
The hard technical point done in this section is to work out the braiding matrix for these blocks. Later, in section 4.3, we prove a pentagon identity relating braiding matrices for $K$ and $K-1$, which proves by induction that the gauge-theory braiding matrix is the correct one for $W_{N}$ conformal blocks. Again, this approach is not sufficient to prove conjectured expressions of conformal blocks, only their braiding and monodromy behaviour. ${ }^{14}$

\subsection{Braiding a fundamental degenerate}

We focus for now on $\langle\widehat{V} \widehat{V} \widehat{V} \widehat{V}\rangle=\left\langle\widehat{V}_{\alpha_{\infty}}(\infty) \widehat{V}_{(\varkappa+b) h_{1}}(1) \widehat{V}_{-b h_{1}}(x, \bar{x}) \widehat{V}_{\alpha_{0}}(0)\right\rangle$, a four-point correlation function with one semi-degenerate momentum $(\varkappa+b) h_{1}$ and a degenerate $-b h_{1}$, labelled by the highest weight $h_{1}$ of the fundamental representation of $A_{N-1}$. The shift by $b$ in $\varkappa$ simplifies some expressions. Momenta are otherwise generic.

Our discussion relies on two fusion rules [53] which can be obtained for the Toda CFT using the Coulomb gas formalism:

$$
\begin{aligned}
\widehat{V}_{-b h_{1}} \times \widehat{V}_{\alpha} & =\sum_{i=1}^{N}\left[\widehat{V}_{\alpha-b h_{i}}\right] \\
\widehat{V}_{-b h_{1}} \times \widehat{V}_{(\varkappa+b) h_{1}} & =\left[\widehat{V}_{\varkappa h_{1}}\right]+\left[\widehat{V}_{(\varkappa+b) h_{1}-b h_{2}}\right],
\end{aligned}
$$

where $\left[\widehat{V}_{\ldots .}\right]$ denotes contributions from $W_{N}$ descendants of a primary operator with the given momentum. As usual in $2 \mathrm{~d}$ CFT, the four-point function can be expanded in three different channels by taking the OPE of $\widehat{V}_{-b h_{1}}$ with any of the three other operators. The fusion rules restrict the internal momentum to $\alpha_{0}-b h_{s}$ for $1 \leq s \leq N$ in the s-channel, $\alpha_{\infty}-b h_{s}$ in the u-channel, and $\varkappa h_{1}$ or $(\varkappa+b) h_{1}-b h_{2}$ in the t-channel.

In the s-channel, the four-point function is a sum of $N$ factorized terms:

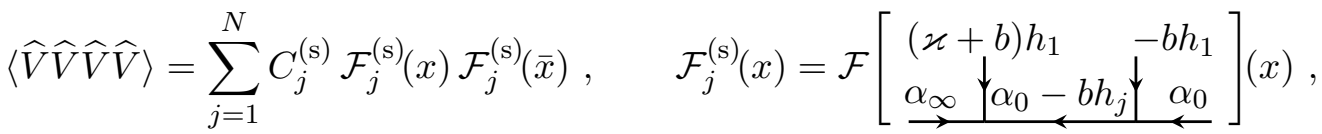

$$
\begin{aligned}
& C_{j}^{(\mathrm{s})}=\frac{\left\langle\widehat{V}_{\alpha_{\infty}} \widehat{V}_{(\varkappa+b) h_{1}} \widehat{V}_{\alpha_{0}-b h_{j}}\right\rangle\left\langle\widehat{V}_{2 Q-\alpha_{0}+b h_{j}} \widehat{V}_{-b h_{1}} \widehat{V}_{\alpha_{0}}\right\rangle}{\left\langle\widehat{V}_{\alpha_{0}-b h_{j}} \widehat{V}_{2 Q-\alpha_{0}+b h_{j}}\right\rangle},
\end{aligned}
$$

where we have absorbed all of the position dependence in the conformal blocks $\mathcal{F}$. A useful property of conformal blocks is their $x \rightarrow 0$ expansion

$$
\begin{aligned}
\mathcal{F}_{j}^{(\mathrm{s})}(x) & =x^{\Delta\left(\alpha_{0}-b h_{j}\right)-\Delta\left(\alpha_{0}\right)-\Delta\left(-b h_{1}\right)}(1+\cdots) \\
& =x^{b\left\langle\alpha_{0}-Q, h_{j}\right\rangle+\frac{N-1}{2}\left(b^{2}+1\right)}(1+\cdots)
\end{aligned}
$$

where $(1+\cdots)$ is a series in non-negative integer powers of $x$, and similarly for $\mathcal{F}_{j}^{(\mathrm{s})}(\bar{x})$. Because of radial ordering, the functions $\mathcal{F}_{j}^{(\mathrm{s})}$ are a priori only defined on the unit disk (with a branch point at 0 ), but since $\langle\widehat{V} \widehat{V} \widehat{V} \widehat{V}\rangle$ is smooth away from 0,1 , and $\infty$ the functions can be analytically continued to any simply connected domain avoiding these points. Two

\footnotetext{
${ }^{14}$ We thank Sylvain Ribault for discussions on this topic. It also seems feasible to use the recent development [52] to prove our conjectured conformal blocks.
} 
natural choices are the complex plane minus cuts on $(-\infty, 0] \cup[1, \infty)$, and the complex plane minus cuts on $[0,1] \cup[1, \infty)$. We will mostly use the second one.

The u-channel decomposition is similar, and conformal blocks have a simple $x \rightarrow \infty$ expansion in terms of a series $1+\cdots$ with non-negative integer powers of $1 / x$ :

$$
\begin{aligned}
\langle\widehat{V} \widehat{V} \widehat{V} \widehat{V}\rangle & =\sum_{j=1}^{N} C_{j}^{(\mathrm{u})} \mathcal{F}_{j}^{(\mathrm{u})}(x) \mathcal{F}_{j}^{(\mathrm{u})}(\bar{x}) \\
\mathcal{F}_{j}^{(\mathrm{u})}(x) & =x^{\Delta\left(\alpha_{\infty}\right)-\Delta\left(\alpha_{\infty}-b h_{j}\right)-\Delta\left(-b h_{1}\right)}(1+\cdots) \\
& =x^{-b\left\langle\alpha_{\infty}-Q, h_{j}\right\rangle+\frac{N-1}{2}\left(b^{2}+1\right)+\frac{N-1}{N} b^{2}}(1+\cdots) .
\end{aligned}
$$

Again, $\mathcal{F}_{j}^{(\mathrm{u})}(x)$ extends to $\mathbb{C} \backslash\{0,1\}$ minus some cuts, for instance along $[0,1] \cup[1, \infty)$.

The t-channel is more subtle, as it involves three-point functions of $\widehat{V}_{\alpha_{\infty}}, \widehat{V}_{\alpha_{0}}$, and a descendant of the primary $\widehat{V}_{\varkappa h_{1}}$ or $\widehat{V}_{(\varkappa+b) h_{1}-b h_{2}}$. Contributions from primaries with momentum $\varkappa h_{1}$ factorize because this momentum is semidegenerate. Contributions from primaries with momentum $(\varkappa+b) h_{1}-b h_{2}$ do not factorize (except for $N=2$ ). We deduce

$$
\langle\widehat{V} \widehat{V} \widehat{V} \widehat{V}\rangle=C_{1}^{(\mathrm{t})} \mathcal{F}_{1}^{(\mathrm{t})}(x) \mathcal{F}_{1}^{(\mathrm{t})}(\bar{x})+C_{2}^{(\mathrm{t})} \mathbb{F}_{2}^{(\mathrm{t})}(x, \bar{x})
$$

with the following $x \rightarrow 1$ expansions, where $1+\cdots$ denote series in non-negative integer powers of $(1-x)$ and $(1-\bar{x})$,

$$
\begin{aligned}
\mathcal{F}_{1}^{(\mathrm{t})}(x) \mathcal{F}_{1}^{(\mathrm{t})}(\bar{x}) & =|1-x|^{2\left[\Delta\left(\varkappa h_{1}\right)-\Delta\left((\varkappa+b) h_{1}\right)-\Delta\left(-b h_{1}\right)\right]}(1+\cdots) \\
& =|1-x|^{2 b(\varkappa+b)(N-1) / N}(1+\cdots) \\
\mathbb{F}_{2}^{(\mathrm{t})}(x, \bar{x}) & =|1-x|^{2\left[\Delta\left((\varkappa+b) h_{1}-b h_{2}\right)-\Delta\left((\varkappa+b) h_{1}\right)-\Delta\left(-b h_{1}\right)\right]}(1+\cdots) \\
& =|1-x|^{2\left[-b(\varkappa+b) / N+b^{2}+1\right]}(1+\cdots) .
\end{aligned}
$$

\subsubsection{Monodromy matrices}

Away from the cuts, the equality $\sum_{j} C_{j}^{(\mathrm{s})}\left|\mathcal{F}_{j}^{(\mathrm{s})}\right|^{2}=\sum_{j} C_{j}^{(\mathrm{u})}\left|\mathcal{F}_{j}^{(\mathrm{u})}\right|^{2}$ implies that the sets $\left\{\mathcal{F}_{j}^{(\mathrm{u})}\right\}$ and $\left\{\mathcal{F}_{k}^{(\mathrm{s})}\right\}$ are different bases of an $N$-dimensional space of holomorphic functions. The change of basis matrix is called the braiding matrix. Similarly $\mathbb{F}_{2}^{(\mathrm{t})}$ is (non-canonically) a sum of $N-1$ factorized terms, and their $N-1$ holomorphic factors together with $\mathcal{F}_{1}^{(\mathrm{t})}$ form another basis of the same space.

The expansions of conformal blocks at 0,1 , and $\infty$ above imply certain monodromy properties when analytically continuing the functions through cuts. The monodromy $M_{(0)}$ around $x=0$ is diagonal in the basis $\mathcal{F}^{(\mathrm{s})}$ and eigenvalues can be read off from the expansion (3.5). While the monodromies $M_{(1)}$ and $M_{(\infty)}$ are non-diagonal $N \times N$ matrices in this s-channel basis, they are diagonalizable (by going to the $\mathrm{t}$ - or $\mathrm{u}$-channel bases) and their eigenvalues can be read off from the expansions above. Namely, eigenvalues of the monodromy $M_{(1)}$ around $x=1$ are seen from (3.9) and (3.10), the second one having multiplicity $N-1$, and the monodromy $M_{(\infty)}$ has $N$ eigenvalues known from (3.7). Finally, $M_{(\infty)}=M_{(1)} M_{(0)}$ since $x \in\{0,1, \infty\}$ are the only singular points. 
We thus have a triplet of invertible diagonalizable $N \times N$ matrices $M_{(0)}, M_{(1)}$ and $M_{(\infty)}$ obeying $M_{(\infty)}=M_{(1)} M_{(0)}$ and with prescribed eigenvalues, such that $M_{(1)}$ has eigenvalues $y_{1}$ with multiplicity 1 and $y_{2}$ with multiplicity $N-1$ while eigenvalues of $M_{(0)}$ and $M_{(\infty)}$ have no multiplicity. We now show that such a triplet is unique up to conjugation. Choose a basis where $M_{(0)}$ is diagonal. Since $M_{(1)}-y_{2}$ has rank 1 it can be written as $M_{(1) i j}=y_{2} \delta_{i j}+\left(y_{1}-y_{2}\right) v_{i} w_{j}$ for some vectors $v$ and $w$. By rescaling the $i$-th basis vector by $\sqrt{v_{i} / w_{i}}$ and setting $u_{i}=\sqrt{v_{i} w_{i}}$ we get a basis in which $M_{(0)}=\operatorname{diag}\left(x_{1}, \ldots, x_{N}\right)$ is still diagonal and

$$
M_{(1) i j}=y_{2} \delta_{i j}+\left(y_{1}-y_{2}\right) u_{i} u_{j} .
$$

Denote eigenvalues of $M_{(\infty)}$ as $z_{i}$. Compute the determinant of $M_{(1)}-y_{2} x_{p} M_{(0)}^{-1}$ for some $p$. On the one hand the matrix is the sum of a diagonal matrix $y_{2}\left(1-x_{p} M_{(0)}^{-1}\right)$ with $p$-th entry 0 and a rank 1 matrix so subtracting the $p$-th row then the $p$-th column from others makes it diagonal. On the other hand the matrix is equal to $\left(M_{(\infty)}-y_{2} x_{p}\right) M_{(0)}^{-1}$. We get

$$
\begin{aligned}
\operatorname{det}\left(M_{(1)}-y_{2} x_{p} M_{(0)}^{-1}\right) & =\left(y_{1}-y_{2}\right) u_{p}^{2} \prod_{s \neq p}^{N}\left(y_{2}\left(1-x_{p} x_{s}^{-1}\right)\right) \\
& =\operatorname{det}\left(M_{(\infty)}-y_{2} x_{p}\right) \operatorname{det}\left(M_{(0)}\right)^{-1}=\prod_{s=1}^{N} \frac{z_{s}-y_{2} x_{p}}{x_{s}} .
\end{aligned}
$$

Therefore,

$$
u_{p}^{2}=\frac{\prod_{s=1}^{N}\left(z_{s}-y_{2} x_{p}\right)}{y_{2}^{N-1}\left(y_{1}-y_{2}\right) x_{p} \prod_{s \neq p}^{N}\left(x_{s}-x_{p}\right)} .
$$

This fixes components of $u$ up to signs, which can be absorbed in a choice of basis, hence it fixes $M_{(1)}$ and concludes this straightforward proof of uniqueness.

Armed with this set of monodromy matrices we should ask whether they fix conformal blocks uniquely. The answer is no, of course, as multiplying conformal blocks by a meromorphic function does not change their monodromy. However, we can use more information: the four-point function should only be singular at $z \in\{0,1, \infty\}$ so conformal blocks must be holomorphic (with branch cuts) away from these points.

\subsubsection{Explicit conformal blocks (proof)}

The s-channel conformal blocks proposed in [53] are

$$
\begin{aligned}
\mathcal{F}_{j}^{(\mathrm{s}), \mathrm{FL}}(x)= & x^{b\left\langle\alpha_{0}-Q, h_{j}\right\rangle+\frac{N-1}{2}\left(b^{2}+1\right)}(1-x)^{b^{2}+1-b(\varkappa+b) / N} \\
& \times{ }_{N} F_{N-1}\left(\begin{array}{c}
1-b \varkappa / N+b\left\langle\alpha_{0}-Q, h_{j}\right\rangle+b\left\langle\alpha_{\infty}-Q, h_{k}\right\rangle, 1 \leq k \leq N \\
1+b\left\langle\alpha_{0}-Q, h_{j}-h_{k}\right\rangle, k \neq j
\end{array} \mid x\right),
\end{aligned}
$$

where the hypergeometric function ${ }_{N} F_{N-1}$ is defined in terms of Pochhammer symbols $(a)_{k}=\Gamma(a+k) / \Gamma(a)$ by the series

$$
F\left(\begin{array}{ccc}
a_{1} & \cdots & a_{N} \\
b_{1} & \cdots & b_{N-1}
\end{array} \mid x\right)=\sum_{k \geq 0} \frac{x^{k}}{k !} \frac{\left(a_{1}\right)_{k} \cdots\left(a_{N}\right)_{k}}{\left(b_{1}\right)_{k} \cdots\left(b_{N-1}\right)_{k}} .
$$


They have the correct leading behaviour (3.5) near $x=0$, hence the correct monodromies $M_{(0)}^{\mathrm{FL}}=M_{(0)}$. As we review in section 3.1.3 their analytic continuations close to $x=1$ and $x=\infty$ are known [55] and involve the correct powers of $1-x$ and of $1 / x$, respectively, which means that the monodromy matrices have the correct eigenvalues. Given our uniqueness proof above, all three monodromy matrices (expressed for instance in the s-channel basis) are correct.

Using Fuchsian techniques inspired by [52], let us now deduce that the proposed blocks (3.15) are indeed the correct conformal blocks. ${ }^{15}$ Hypergeometric functions famously obey a differential equation, and in fact the $N$ functions $\mathcal{F}_{j}^{(\mathrm{s}), \mathrm{FL}}$ are linearly-independent solutions to the same $N$-th order Fuchsian differential equation in $x$, regular except for singularities at 0,1 and $\infty$. Thus, the solutions have Wronskian $\operatorname{det} \Phi \neq 0$ away from these singularities, where ${ }^{16}$

$$
\Phi=\left(\begin{array}{cccc}
\mathcal{F}_{1}^{(\mathrm{s}), \mathrm{FL}} & \partial_{x} \mathcal{F}_{1}^{(\mathrm{s}), \mathrm{FL}} & \ldots & \partial_{x}^{N-1} \mathcal{F}_{1}^{(\mathrm{s}), \mathrm{FL}} \\
\vdots & \vdots & & \vdots \\
\mathcal{F}_{N}^{(\mathrm{s}), \mathrm{FL}} & \partial_{x} \mathcal{F}_{N}^{(\mathrm{s}), \mathrm{FL}} & \ldots & \partial_{x}^{N-1} \mathcal{F}_{N}^{(\mathrm{s}), \mathrm{FL}}
\end{array}\right)
$$

Each of the columns has the same monodromy matrices $M_{(0)}, M_{(1)}, M_{(\infty)}$ as the real conformal blocks $\mathcal{F}_{j}^{(\mathrm{s})}$. Now consider the vector $\Phi^{-1}\left(\mathcal{F}_{1}^{(\mathrm{s})}, \ldots, \mathcal{F}_{N}^{(\mathrm{s})}\right)^{T}$. It is holomorphic away from $x=0, x=1, x=\infty$ because $\Phi$ is invertible, and it has no monodromy around these three points because the monodromy matrices cancel. Expanding near $x=0$, the non-integer powers $x^{-\beta_{j}}$ with $\beta_{j}=-b\left\langle\alpha_{0}-Q, h_{j}\right\rangle-\frac{N-1}{2}\left(b^{2}+1\right)$ cancel and we find

$$
\begin{aligned}
\Phi^{-1}\left(\begin{array}{c}
\mathcal{F}_{1}^{(\mathrm{s})} \\
\vdots \\
\mathcal{F}_{N}^{(\mathrm{s})}
\end{array}\right) & =\left(\begin{array}{cccc}
(1+O(x)) & \frac{-\beta_{1}}{x}(1+O(x)) & \ldots & \frac{\left(-\beta_{1}\right)_{N}}{x^{N-1}}(1+O(x)) \\
\vdots & \vdots & \vdots \\
(1+O(x)) & \frac{-\beta_{N}}{x}(1+O(x)) & \ldots & \frac{\left(-\beta_{N}\right)_{N}}{x^{N-1}}(1+O(x))
\end{array}\right)^{-1}\left(\begin{array}{c}
1+O(x) \\
\vdots \\
1+O(x)
\end{array}\right) \\
& =\left(\begin{array}{cccc}
1 & 0 & \cdots & 0 \\
0 & x & \ddots & \vdots \\
\vdots & \ddots & \ddots & 0 \\
0 & \cdots & 0 & x^{N-1}
\end{array}\right)\left(\left(\begin{array}{cccc}
1 & -\beta_{1} & \ldots & \left(-\beta_{1}\right)_{N} \\
\vdots & \vdots & \vdots \\
1 & -\beta_{N} & \ldots & \left(-\beta_{N}\right)_{N}
\end{array}\right)\left(\begin{array}{c}
1 \\
\vdots \\
1
\end{array}\right)+O(x)\right)=\left(\begin{array}{c}
1 \\
0 \\
\vdots \\
0
\end{array}\right)+O(x) .
\end{aligned}
$$

The expansion near 1 (resp. $\infty$ ) works similarly: the columns with derivatives involve different powers of $1-x$ (resp. $1 / x$ ) but this can be factored, leaving a finite result that tends to the column vector $(1,0, \ldots, 0)^{T}$.

Altogether we have a vector whose components are holomorphic on the whole Riemann sphere, hence are constant. We know its value at $x=0$ from (3.18) and deduce that the true conformal blocks are equal to the first column of $\Phi$, namely the expression found in [53].

\footnotetext{
${ }^{15}$ Our argument in version 1 was flawed: treating conformal blocks all together as in [52] is essential. The only assumption in the proof we give now is that the well-known fusion rules (3.1) and (3.2) hold.

${ }^{16}$ Note that each column of the analogue of $\Phi$ in [52] is a collection of blocks for a given value of $\alpha_{\infty}$. We could have done the same, with $\alpha_{\infty}=\alpha-h_{m} / b$ for the $m$-th column where $\alpha$ is some fixed momentum.
} 


\subsubsection{Braiding matrices}

Let $f_{j}^{(\mathrm{s})}$ denote the hypergeometric function in (3.15). We now derive the braiding matrix for the functions $(-x)^{b\left\langle\alpha_{0}-Q, h_{j}\right\rangle} f_{j}^{(\mathrm{s})}(x)$, then convert it to the braiding matrix (3.24) for the blocks $\mathcal{F}$ by introducing phases.

To compute their braiding matrix it is convenient to introduce notations. Let $\mathrm{i} m_{p}$ and $\mathrm{i} \widetilde{m}_{p}$ be $2 N$ complex numbers such that

$$
\alpha_{0}=Q-\frac{1}{b} \sum_{p=1}^{N} \mathrm{i} m_{p} h_{p}, \quad \alpha_{\infty}=Q-\frac{1}{b} \sum_{p=1}^{N} \mathrm{i} \widetilde{m}_{p} h_{p}, \quad \varkappa=\frac{1}{b} \sum_{p=1}^{N}\left(1+\mathrm{i} m_{p}+\mathrm{i} \widetilde{m}_{p}\right) .
$$

This parametrization is redundant under shifts of all $\mathrm{i} m_{p}$ and $-\mathrm{i} \widetilde{m}_{p}$. The s-channel factor $(-x)^{-\mathrm{i} m_{j}} f_{j}^{(\mathrm{s})}(x)$ can be expressed as the Mellin-Barnes integral given below, which converges away from the positive real axis. For $|x| \lessgtr 1$ we can close the contour integral towards $v \rightarrow \pm \infty$, and enclose either the poles at $v+\mathrm{i} m_{j} \in \mathbb{Z}_{\geq 0}$ or the $N$ families of poles at $v-\mathrm{i} \widetilde{m}_{k} \in \mathbb{Z}_{\leq 0}$ labelled by $1 \leq k \leq N$. The first choice yields a single s-channel factor, while the second yields a sum of $N$ u-channel factors:

$$
\begin{aligned}
(-x)^{-\mathrm{i} m_{j}} f_{p}^{(\mathrm{s})}(x) & \stackrel{\text { cont }}{=} D_{j} \int_{-\mathrm{i} \infty}^{\mathrm{i} \infty} \frac{\mathrm{d} v}{2 \pi \mathrm{i}} \frac{\prod_{k=1}^{N} \Gamma\left(-\mathrm{i} \widetilde{m}_{k}+v\right)}{\prod_{k \neq j}^{N} \Gamma\left(1+\mathrm{i} m_{k}+v\right)} \Gamma\left(-v-\mathrm{i} m_{j}\right)(-x)^{v} \\
& \stackrel{\text { cont }}{=} \sum_{k=1}^{N} D_{j} \check{B}_{j k}^{0} \widetilde{D}_{k}(-x)^{\mathrm{i} \widetilde{m}_{k}} f_{k}^{(\mathrm{u})}(x)
\end{aligned}
$$

We will not need the explicit expression for $f_{k}^{(\mathrm{u})}$, which are hypergeometric series $1+\cdots$ in non-positive integer powers of $x$. The coefficients $D, \check{B}^{0}$ and $\widetilde{D}$ are given by

$$
\begin{aligned}
\check{B}_{j k}^{\epsilon} & =\frac{\pi e^{\pi \epsilon\left(m_{j}+\widetilde{m}_{k}\right)}}{\sin \pi\left(-\mathrm{i} \widetilde{m}_{k}-\mathrm{i} m_{j}\right)} \\
D_{j} & =\prod_{t=1}^{N} \frac{\Gamma\left(1+\mathrm{i} m_{t}-\mathrm{i} m_{j}\right)}{\Gamma\left(-\mathrm{i} \widetilde{m}_{t}-\mathrm{i} m_{j}\right)} \quad \widetilde{D}_{k}=\frac{\prod_{t \neq k}^{N} \Gamma\left(-\mathrm{i} \widetilde{m}_{t}+\mathrm{i} \widetilde{m}_{k}\right)}{\prod_{t=1}^{N} \Gamma\left(1+\mathrm{i} m_{t}+\mathrm{i} \widetilde{m}_{k}\right)}
\end{aligned}
$$

Here we have included a parameter $\epsilon \in\{0, \pm 1\}$. It is sometimes convenient to consider schannel factors $x^{-\mathrm{i} m_{j}} f_{j}^{(\mathrm{s})}(x)$ analytically continued with branch cuts on $(-\infty, 0] \cup[1,+\infty)$, and u-channel factors $x^{\mathrm{i} \widetilde{m}_{k}} f_{k}^{(\mathrm{u})}(x)$ with branch cuts along $(-\infty, 0] \cup[0,1]$. Using $(-x)^{\lambda}=$ $e^{-\mathrm{i} \pi \epsilon \lambda} x^{\lambda}$ for $\epsilon=\operatorname{sign}(\operatorname{Im} x)$, we obtain

$$
x^{-\mathrm{i} m_{j}} f_{j}^{(\mathrm{s})}(x) \stackrel{\text { cont }}{=} \sum_{k=1}^{N} D_{j} \check{B}_{j k}^{\epsilon} \widetilde{D}_{k} x^{\mathrm{i} \widetilde{m}_{k}} f_{k}^{(\mathrm{u})}(x) .
$$


The braiding matrix in $\mathcal{F}_{j}^{(\mathrm{s})}(x)=\sum_{k=1}^{N} \mathbf{B}_{j k}^{\epsilon} \mathcal{F}_{k}^{(\mathrm{u})}(x)$ includes a phase: explicitly we get

$$
\begin{aligned}
\mathbf{B}_{j k}^{\epsilon}\left[\begin{array}{cc}
(\varkappa+b) h_{1} & -b h_{1} \\
\alpha_{\infty} & \alpha_{0}
\end{array}\right]= & e^{\mathrm{i} \pi \epsilon\left[b(\varkappa+b) / N-b^{2}-1\right]} D_{j} \check{B}_{j k}^{\epsilon} \widetilde{D}_{k} \\
= & \frac{\prod_{t \neq j}^{N} \Gamma\left(1+b\left\langle Q-\alpha_{0}, h_{t}-h_{j}\right\rangle\right)}{\prod_{u=1}^{N} \Gamma\left(1-\frac{b \varkappa}{N}-b\left\langle Q-\alpha_{0}, h_{j}\right\rangle-b\left\langle Q-\alpha_{\infty}, h_{u}\right\rangle\right)} \\
& \times \frac{\pi e^{\mathrm{i} \pi \epsilon\left[-b^{2}(N-1) / N-b\left\langle Q-\alpha_{0}, h_{j}\right\rangle-b\left\langle Q-\alpha_{\infty}, h_{k}\right\rangle\right]}}{\sin \pi\left(1-\frac{b \varkappa}{N}-b\left\langle Q-\alpha_{0}, h_{j}\right\rangle-b\left\langle Q-\alpha_{\infty}, h_{k}\right\rangle\right)} \\
& \times \frac{\prod_{u \neq k}^{N} \Gamma\left(b\left\langle Q-\alpha_{\infty}, h_{k}-h_{u}\right\rangle\right)}{\prod_{t=1}^{N} \Gamma\left(\frac{b \varkappa}{N}+b\left\langle Q-\alpha_{0}, h_{t}\right\rangle+b\left\langle Q-\alpha_{\infty}, h_{k}\right\rangle\right)} .
\end{aligned}
$$

The s-channel blocks $\mathcal{F}_{j}^{(\mathrm{s})}(x)$ have the expected power of $x$ (hence the expected monodromies) around 0 . The eigenvalues of the monodromy $M_{(\infty)}$ around $\infty$ are also correct, as can be checked by combining the $\mathrm{u}$-channel factors in (3.21) with the additional powers of $x$ and $1-x$ in (3.15) and comparing to the $\mathrm{u}$-channel asymptotics (3.7) expected from CFT. Finally, from the braiding matrix above we deduce the monodromy $M_{(1)}=\mathbf{B}^{+}\left(\mathbf{B}^{-}\right)^{-1}$ around 1. All components $\check{B}_{j k}^{+}-\check{B}_{j k}^{-}=2 \pi \mathrm{i}$ are equal hence $\check{B}^{+}-\check{B}^{-}$has rank 1 , and $e^{-2 \pi \mathrm{i} \gamma_{1}} M_{(1)}-1=D\left(\check{B}^{+}-\check{B}^{-}\right) \widetilde{D}\left(\mathbf{B}^{-}\right)^{-1}$ too. Therefore, $M_{(1)}$ has the eigenvalue $e^{2 \pi \mathrm{i} \gamma_{1}}$ with multiplicity $N-1$. Its last eigenvalue is fixed by $\operatorname{det} e^{-2 \pi \mathrm{i} \gamma_{1}} M_{(1)}=\operatorname{det} \check{B}^{+} / \operatorname{det} \check{B}^{-}$. These eigenvalues coincide with those expected of t-channel blocks. In fact the precise exponents of $(1-x)$ also match [55]. We used this in our proof that conformal blocks are given by the explicit hypergeometric formula (3.15).

\subsection{Braiding a symmetric degenerate}

We now generalize the discussion above to a degenerate vertex operator $\widehat{V}_{-K b h_{1}}$ labelled by the $K$-th symmetric representation $\mathcal{R}\left(K h_{1}\right)$ of $A_{N-1}$. Namely we consider its fourpoint function with two generic operators $\widehat{V}_{\alpha_{\infty}}$ and $\widehat{V}_{\alpha_{0}}$ and one semi-degenerate $\widehat{V}_{(\varkappa+K b) h_{1}}$, including a shift by $K b$ for later convenience. The braiding (3.42) we find is new. Contrarily to the $K=1$ case of the previous section which we reuse throughout the paper, the $K>1$ case is only used to find the correct generalization to continuous values of $K$ in section 4 .

Explicit s-channel and u-channel decompositions and their conformal blocks were conjectured in [54, appendix A.5], thanks to a relation with sphere partition functions of $2 \mathrm{~d}$ $\mathcal{N}=(2,2)$ gauge theories. We use the same notations $\mathrm{i} m_{p}$ and $\mathrm{i} \widetilde{m}_{p}$ as in (3.19) (ambiguous under overall shifts of all $\mathrm{i} m_{p}$ and $\left.-\mathrm{i} \widetilde{m}_{p}\right)$, namely $\alpha_{0}=Q-\frac{1}{b} \sum_{p=1}^{N} \mathrm{i} m_{p} h_{p}$ and $\alpha_{\infty}=Q-\frac{1}{b} \sum_{p=1}^{N} \mathrm{i} \widetilde{m}_{p} h_{p}$ and $\varkappa=\frac{1}{b} \sum_{p=1}^{N}\left(1+\mathrm{i} m_{p}+\mathrm{i} \widetilde{m}_{p}\right)$. The four-point function is a sum over weights $h_{[n]}=\sum_{s=1}^{N} n_{s} h_{s}$ of the symmetric representation $\mathcal{R}\left(K h_{1}\right)$ : up to a constant $C$,

$$
\begin{aligned}
& \left\langle\widehat{V}_{\alpha_{\infty}}(\infty) \widehat{V}_{(\varkappa+K b) h_{1}}(1) \widehat{V}_{-K b h_{1}}(x, \bar{x}) \widehat{V}_{\alpha_{0}}(0)\right\rangle \\
& \quad=C \sum_{n_{1}+\cdots+n_{N}=K}\left[\prod_{(s, \mu)}^{[n]} \prod_{t=1}^{N} \frac{\gamma\left(\mathrm{i} m_{s \mu}-\mathrm{i} m_{t n_{t}}\right)}{\gamma\left(1+\mathrm{i} \widetilde{m}_{t}+\mathrm{i} m_{s \mu}\right)} \mathcal{F}_{[n]}^{(\mathrm{s})}(x) \mathcal{F}_{[n]}^{(\mathrm{s})}(\bar{x})\right]
\end{aligned}
$$


where $\gamma(y)=\Gamma(y) / \Gamma(1-y)$ and we introduced the notations

$$
\prod_{(s, \mu)}^{[n]}=\prod_{s=1}^{N} \prod_{\mu=0}^{n_{s}-1} \text { and } \quad \mathrm{i} m_{s \mu}=\mathrm{i} m_{s}+\mu b^{2} .
$$

The conformal blocks $\mathcal{F}_{[n]}^{(\mathrm{s})}$ are

$$
\begin{aligned}
\mathcal{F}_{[n]}^{(\mathrm{s})}(x) & =(1-x)^{-\gamma_{1}} x^{-\gamma_{0}-\sum_{(s, \mu)}^{[n]} \mathrm{i} m_{s \mu}} f_{[n]}^{(\mathrm{s})}(x) \\
\gamma_{0} & =-\frac{K(K-1)}{2} b^{2}-\frac{K(N-1)}{2}\left(b^{2}+1\right)-\frac{K}{N} \sum_{s=1}^{N} \mathrm{i} m_{s} \\
\gamma_{1} & =-\frac{K(N-K)}{N} b^{2}+\frac{K}{N} \sum_{s=1}^{N}\left(\mathrm{i} m_{s}+\mathrm{i} \widetilde{m}_{s}\right) \\
f_{[n]}^{(\mathrm{s})}(x) & =\sum_{k \ldots \geq 0} \prod_{(s, \mu)}^{[n]}\left[x^{k_{s \mu}} \prod_{t=1}^{N} \frac{\left(-\mathrm{i} \widetilde{m}_{t}-\mathrm{i} m_{s \mu}\right)_{k_{s \mu}}}{\left(1+\mathrm{i} m_{t n_{t}}-\mathrm{i} m_{s \mu}\right)_{k_{s \mu}}}\right. \\
& \left.\times \frac{\prod_{t=1}^{N}\left(1+\mathrm{i} m_{t n_{t}}-\mathrm{i} m_{s \mu}+k_{s \mu}-k_{t\left(n_{t}-1\right)}\right)_{\left.k_{t(n t}-1\right)}}{\prod_{(t, \nu)}^{[n]}\left(1+\mathrm{i} m_{t \nu}-\mathrm{i} m_{s \mu}+k_{s \mu}-k_{t \nu}\right)_{k_{t \nu}-k_{t(\nu-1)}}}\right] .
\end{aligned}
$$

For a given weight $h_{[n]}$ of $\mathcal{R}\left(K h_{1}\right)$, and a choice of $1 \leq p \leq N$ we consider the following generalization of the Mellin-Barnes integral (3.21) used for $K=1$.

$$
\begin{aligned}
I_{[n]}^{p}= & \frac{\Gamma\left(-b^{2}\right)^{K}}{K !} \prod_{j=1}^{K}\left[\int_{-\infty}^{\infty} \frac{\mathrm{d} \sigma_{j}}{2 \pi}\right]\left\{\prod_{i \neq j}^{K} \frac{\Gamma\left(\mathrm{i} \sigma_{i}-\mathrm{i} \sigma_{j}-b^{2}\right)}{\Gamma\left(\mathrm{i} \sigma_{i}-\mathrm{i} \sigma_{j}\right)}\right. \\
& \left.\times \prod_{j=1}^{K}\left[(-x)^{\mathrm{i} \sigma_{j}} \frac{\prod_{s=1}^{N}\left[\Gamma\left(-\mathrm{i} \widetilde{m}_{s}+\mathrm{i} \sigma_{j}\right) \Gamma\left(-\mathrm{i} m_{s}-\mathrm{i} \sigma_{j}\right)\right]}{\prod_{s \neq p}^{N}\left[\Gamma\left(1+\mathrm{i} m_{s n_{s}}+\mathrm{i} \sigma_{j}\right) \Gamma\left(-\mathrm{i} m_{s n_{s}}-\mathrm{i} \sigma_{j}\right)\right]}\right]\right\} .
\end{aligned}
$$

The contours lie between poles of all the $\Gamma\left(-\mathrm{i} \widetilde{m}_{s}+\mathrm{i} \sigma_{j}\right)$ and poles of all the $\Gamma\left(-\mathrm{i} m_{s}-\mathrm{i} \sigma_{j}\right)$. The integral converges for $x \notin[0, \infty)$ because there are fewer $\Gamma$ functions in the denominator.

For $|x| \lessgtr 1$ we can close contours towards $\mathrm{i} \sigma_{j} \rightarrow \mp \mathrm{i} \infty$, enclosing some poles. Start with $|x|<1$. If the partition $[n]$ has a single non-zero entry (e.g., if $K=1$ ) we can choose $p$ so that $n_{s}=0$ for $s \neq p$, and then all numerators $\Gamma\left(-i m_{s}-i \sigma_{j}\right)$ cancel with denominators except for $s=p$. But in general there is no such cancellation and the integral gives a linear combination of s-channel factors (3.30); our whole work will be to disentangle this sum:

$$
\begin{aligned}
I_{[n]}^{p} & =\sum_{[k]} T_{[n][k]}^{p}(-x)^{-\sum_{(s, \mu)}^{[k]} \mathrm{i} m_{s \mu}} f_{[k]}^{(\mathrm{s})}, \\
T_{[n][k]}^{p} & =\prod_{(s, \mu)}^{[k]} \frac{\prod_{t=1}^{N}\left[\Gamma\left(-\mathrm{i} \widetilde{m}_{t}-\mathrm{i} m_{s \mu}\right) \Gamma\left(\mathrm{i} m_{s \mu}-\mathrm{i} m_{t k_{t}}\right)\right]}{\prod_{t \neq p}^{N}\left[\Gamma\left(1+\mathrm{i} m_{t n_{t}}-\mathrm{i} m_{s \mu}\right) \Gamma\left(\mathrm{i} m_{s \mu}-\mathrm{i} m_{t n_{t}}\right)\right]} .
\end{aligned}
$$

The sum ranges over weights of $\mathcal{R}\left(K h_{1}\right)$, but the matrix $T^{p}$ is "triangular" in the sense that its component $T_{[n][k]}^{p}$ vanishes if $n_{s}<k_{s}$ for any $s \neq p$. 
Closing contours towards $\mathrm{i} \sigma_{j} \rightarrow \mathrm{i} \infty$ for $|x|>1$ gives a sum of $\mathrm{u}$-channel factors

$$
\begin{aligned}
I_{[n]}^{p} & =\sum_{[\widetilde{n}]} U_{[n][\widetilde{n}]}^{p}(-x)^{\sum_{(s, \mu)}^{[\widetilde{n}]} \mathrm{i} \widetilde{m}_{s \mu}} f_{[\widetilde{n}]}^{(\mathrm{u})} \\
U_{[n][\widetilde{n}]}^{p} & =\prod_{(s, \mu)}^{[\tilde{n}]} \frac{\prod_{t=1}^{N}\left[\Gamma\left(\mathrm{i} \widetilde{m}_{s \mu}-\mathrm{i} \widetilde{m}_{t \widetilde{n} t}\right) \Gamma\left(-\mathrm{i} \widetilde{m}_{s \mu}-\mathrm{i} m_{t}\right)\right]}{\prod_{t \neq p}^{N}\left[\Gamma\left(1+\mathrm{i} m_{t n_{t}}+\mathrm{i} \widetilde{m}_{s \mu}\right) \Gamma\left(-\mathrm{i} \widetilde{m}_{s \mu}-\mathrm{i} m_{t n_{t}}\right)\right]} .
\end{aligned}
$$

This leads to the braiding

$$
(-x)^{-\sum_{(s, \mu)}^{[n]} \mathrm{i} m_{s \mu}} f_{[n]}^{(\mathrm{s})}=\sum_{[\widetilde{n}]}\left(\left(T^{p}\right)^{-1} U^{p}\right)_{[n][\widetilde{n}]}(-x)^{\sum_{(s, \mu)}^{[\tilde{n}]}{ }^{\mathrm{i} \widetilde{m}_{s \mu}}} f_{[\widetilde{n}]}^{(\mathrm{u})} .
$$

We thus need to invert the matrix $T^{p}$ then multiply the result by $U^{p}$. A consistency check is that the braiding matrix $\left(T^{p}\right)^{-1} U^{p}$ must not depend on $p$.

Split $T^{p}=\check{T}^{p} D^{p}$ with $D^{p}$ diagonal:

$$
\begin{aligned}
& \check{T}_{[n][k]}^{p}=\prod_{(s, \mu)}^{[k]} \prod_{t \neq p}^{N} \frac{1}{\pi} \sin \pi\left(\mathrm{i} m_{s \mu}-\mathrm{i} m_{t n_{t}}\right) \\
& D_{[k][l]}^{p}=\delta_{[k][l]} \prod_{(s, \mu)}^{[k]} \prod_{t=1}^{N}\left[\Gamma\left(-\mathrm{i} \widetilde{m}_{t}-\mathrm{i} m_{s \mu}\right) \Gamma\left(\mathrm{i} m_{s \mu}-\mathrm{i} m_{t k_{t}}\right)\right] .
\end{aligned}
$$

A proposal for $\left(\check{T}^{p}\right)^{-1}$ is found by trial and error:

$$
\left(\left(\check{T}^{p}\right)^{-1}\right)_{[n][k]}=\left\{\begin{array}{l}
0 \quad \text { if } n_{t}<k_{t} \text { for any } t \neq p, \text { and otherwise } \\
\frac{\prod_{s<t}^{N}\left[\frac{1}{\pi} \sin \pi\left(\mathrm{i} m_{s k_{s}}-\mathrm{i} m_{t k_{t}}\right) \frac{1}{\pi} \sin \pi\left(\mathrm{i} m_{t n_{t}}-\mathrm{i} m_{s n_{s}}\right)\right]}{\prod_{t \neq p}^{N}\left[\frac{1}{\pi} \sin \pi\left(\mathrm{i} m_{t k_{t}}-\mathrm{i} m_{p k_{p}}\right) \prod_{\substack{1 \leq s \leq N, 0 \leq \mu \leq n_{s} \\
(s, \mu) \neq\left(t, k k_{t}\right)}} \frac{1}{\pi} \sin \pi\left(\mathrm{i} m_{s \mu}-\mathrm{i} m_{t k_{t}}\right)\right]} .
\end{array}\right.
$$

We must prove that $\sum_{[k]}\left(\left(\check{T}^{p}\right)^{-1}\right)_{[n][k]}\left(\check{T}^{p}\right)_{[k][j]}=\delta_{[n][j]}$. Since both matrices are "triangular" their product is as well. It is straightforward to compute the diagonal coefficients $\left(\left(\check{T}^{p}\right)^{-1} \check{T}^{p}\right)_{[n][n]}=\left(\left(\check{T}^{p}\right)^{-1}\right)_{[n][n]}\left(\check{T}^{p}\right)_{[n][n]}=1$. There remains to show that coefficients $[n][j]$ with $j_{s} \leq n_{s}$ for all $s \neq p$, and with $[j] \neq[n]$ (equivalently $j_{p}>n_{p}$ ) vanish. Cancelling factors of $\frac{1}{\pi} \sin \pi(\ldots)$ as much as possible yields

$$
\begin{aligned}
& \sum_{[k]}\left(\left(\check{T}^{p}\right)^{-1}\right)_{[n][k]}\left(\check{T}^{p}\right)_{[k][j]} \\
& =\sum_{\substack{[k] \\
j_{s} \leq k_{s} \leq n_{s} \forall s \neq p}} \prod_{\substack{1 \leq s<t \leq N \\
s, t \neq p}}\left[\frac{1}{\pi} \sin \pi\left(\mathrm{i} m_{s k_{s}}-\mathrm{i} m_{t k_{t}}\right) \frac{1}{\pi} \sin \pi\left(\mathrm{i} m_{t n_{t}}-\mathrm{i} m_{s n_{s}}\right)\right] \\
& \times \prod_{t \neq p}^{N} \frac{\frac{1}{\pi} \sin \pi\left(\mathrm{i} m_{p n_{p}}-\mathrm{i} m_{t n_{t}}\right) \prod_{\substack{j_{p}-1 \\
j_{p}+1}}^{j_{n}} \sin \pi\left(\mathrm{i} m_{p \mu}-\mathrm{i} m_{t k_{t}}\right)}{\prod_{\substack{s \neq p, j_{s} \leq \mu \leq n_{s} \\
(s, \mu) \neq\left(t, k_{t}\right)}} \frac{1}{\pi} \sin \pi\left(\mathrm{i} m_{s \mu}-\mathrm{i} m_{t k_{t}}\right)} .
\end{aligned}
$$


This is the sum of residues of

$$
\begin{aligned}
& \prod_{\substack{s<t \\
s, t \neq p}}^{N}\left[\frac{1}{\pi} \sin \pi\left(\mathrm{i} \tau_{t}-\mathrm{i} \tau_{s}\right) \frac{1}{\pi} \sin \pi\left(\mathrm{i} m_{t n_{t}}-\mathrm{i} m_{s n_{s}}\right)\right] \\
& \times \prod_{t \neq p}^{N} \frac{\frac{1}{\pi} \sin \pi\left(\mathrm{i} m_{p n_{p}}-\mathrm{i} m_{t n_{t}}\right) \prod_{\mu=n_{p}+1}^{j_{p}-1} \frac{1}{\pi} \sin \pi\left(\mathrm{i} m_{p \mu}+\mathrm{i} \tau_{t}\right)}{\prod_{s \neq p}^{N} \prod_{\mu=j_{s}}^{n_{s}} \frac{1}{\pi} \sin \pi\left(\mathrm{i} m_{s \mu}+\mathrm{i} \tau_{t}\right)}
\end{aligned}
$$

at $\mathrm{i} \tau_{t}=-\mathrm{i} m_{t k_{t}}$. Each $\mathrm{i} \tau_{t}$ appears in $N-2+j_{p}-n_{p}-1$ sines in the numerator, and $\sum_{s \neq p}\left(1+n_{s}-j_{s}\right)=N-1+j_{p}-n_{p}$ in the denominator, in other words, two more. Thus the function is 1-periodic in each variable $\mathrm{i} \tau_{t}$, and decays exponentially as $\mathrm{i} \tau_{t} \rightarrow \pm \infty$. The sum of residues thus vanishes, because it is the sum of all residues in a fundamental domain of the periodicity, and there is no contribution from infinity. This establishes (3.39).

The braiding matrix (3.36) is then $B=\left(T^{p}\right)^{-1} U^{p}=\left(D^{p}\right)^{-1}\left(\check{T}^{p}\right)^{-1} U^{p}$. The result is a sum of residues of some function of $N-1$ variables $\tau_{t}$ for $t \neq p$. Relabelling the variables $\tau_{t}$ using a permutation of $\llbracket 1, N \rrbracket$ so that they are numbered from 1 to $N-1$ and $\phi(N)=p$, we obtain (recall the notations (3.19) and (3.26))

$$
\begin{aligned}
B_{[n][\widetilde{n}]}^{\phi}=(-1)^{\phi} & \prod_{t=1}^{N} \frac{\prod_{(s, \mu)}^{[\widetilde{n}]} \Gamma\left(\mathrm{i} \tilde{m}_{s \mu}-\mathrm{i} \widetilde{m}_{t \widetilde{n}_{t}}\right) \Gamma\left(-\mathrm{i} \widetilde{m}_{s \mu}-\mathrm{i} m_{t}\right)}{\prod_{(s, \mu)}^{[n]} \Gamma\left(-\mathrm{i} \widetilde{m}_{t}-\mathrm{i} m_{s \mu}\right) \Gamma\left(\mathrm{i} m_{s \mu}-\mathrm{i} m_{t n_{t}}\right)} \prod_{s<t}^{N} \frac{\sin \pi\left(\mathrm{i} m_{t n_{t}}-\mathrm{i} m_{s n_{s}}\right)}{\pi} \\
& \times \prod_{j=1}^{N-1}\left[\sum_{k_{j}=0}^{n_{\phi(j)}} \underset{\mathrm{i} \tau_{j}=-\mathrm{i} m_{\phi(j) k_{j}}}{\operatorname{res}} \frac{\prod_{(s, \mu)}^{[\widetilde{n}]} \frac{1}{\pi} \sin \pi\left(-\mathrm{i} \widetilde{m}_{s \mu}+\mathrm{i} \tau_{j}\right)}{\prod_{s=1}^{N} \prod_{\mu=0}^{n_{s}} \frac{1}{\pi} \sin \pi\left(\mathrm{i} m_{s \mu}+\mathrm{i} \tau_{j}\right)} \prod_{i=1}^{j-1} \frac{\sin \pi\left(\mathrm{i} \tau_{j}-\mathrm{i} \tau_{i}\right)}{\pi}\right]
\end{aligned}
$$

where $(-1)^{\phi}$ is the signature of $\phi$. This expression does not change if we replace $\phi$ by another permutation such that $\phi(N)=p$ and we permute the $\tau_{j}$ accordingly: indeed, the sign coming from $\sin \pi\left(\mathrm{i} \tau_{j}-\mathrm{i} \tau_{i}\right)$ is compensated by the change in $(-1)^{\phi}$.

Let us show that $B^{\phi}$ does not depend on the arbitrary choice of $p$ either, hence is independent of $\phi$. Choose an index $1 \leq j \leq N-1$. The variable $\tau_{j}$ appears in $N-2+K$ sines in the numerator and $N+K$ sines in the denominator of (3.42). We thus have $\mathrm{i} \tau_{j} \rightarrow \mathrm{i} \tau_{j}+1$ periodicity and no residue at infinity, hence the sum of residues at $\mathrm{i} \tau_{j}=-\mathrm{i} m_{\phi(j) k_{j}}$ is equal to minus the sum of all other residues in a strip of width 1 . This yields a sum over $\mathrm{i} \tau_{j}=-\mathrm{i} m_{\phi(i) k}$ for all $1 \leq i \leq N$ with $i \neq j$ and $0 \leq k \leq n_{\phi(i)}$. The contribution from a given $i$ with $i<N$ (and $i \neq j$ ) vanishes by antisymmetry under the exchange $\tau_{i} \leftrightarrow \tau_{j}$, thus only the poles at $-\mathrm{i} m_{\phi(N) k}=-\mathrm{i} m_{p k}$ contribute. All in all, we obtain the same expression as (3.42), with $\phi(j)$ and $\phi(N)$ exchanged. The sign coming from flipping the contour is absorbed into a change of the signature $(-1)^{\phi}$.

As for $K=1$, the braiding matrix for $\mathcal{F}_{[n]}^{(\mathrm{s})}(x)$ is obtained by including a phase $e^{\mathrm{i} \pi \epsilon \gamma_{1}}$, and another phase comes from using factors $x^{\cdots}$ instead of $(-x)^{\cdots}$. Putting everything together yields

$$
\begin{aligned}
\mathcal{F}_{[n]}^{(\mathrm{s})}(x) & =\sum_{[\widetilde{n}]} \mathbf{B}_{[n][\widetilde{n}]}^{\epsilon} \mathcal{F}_{[\widetilde{n}]}^{(\mathrm{u})}(x) \\
\mathbf{B}_{[n][\widetilde{n}]}^{\epsilon} & =e^{\mathrm{i} \pi \epsilon \gamma_{1}} e^{\mathrm{i} \pi \epsilon \sum_{(s, \mu)}^{[n]}\left(-\mathrm{i} m_{s, \mu}\right)} B_{[n][\widetilde{n}]}^{\phi} e^{-\mathrm{i} \pi \epsilon \sum_{(s, \mu)}^{[\widetilde{n}]} \mathrm{i} \tilde{m}_{s, \mu}} .
\end{aligned}
$$


The explicit expression of $B^{\phi}$ involves a permutation $\phi$, but is independent of it. To translate this expression explicitly back from the $\{\mathrm{i} m, \mathrm{i} \widetilde{m}\}$ notation (3.19) to momenta, replace

$$
\begin{aligned}
& \mathrm{i} m_{s \mu}=b\left\langle Q-\alpha_{0}, h_{s}\right\rangle+\mu b^{2}+\frac{1}{N} \sum_{t=1}^{N} \mathrm{i} m_{t} \\
& \mathrm{i} \widetilde{m}_{s \mu}=\frac{b \varkappa}{N}+b\left\langle Q-\alpha_{\infty}, h_{s}\right\rangle+\mu b^{2}-1-\frac{1}{N} \sum_{t=1}^{N} \mathrm{i} m_{t}
\end{aligned}
$$

then shift the variables $\mathrm{i} \tau_{j}$ to absorb $\frac{1}{N} \sum_{t=1}^{N} \mathrm{i} m_{t}$.

Note that the starting point of this calculation, namely the explicit expression (3.25) for the four-point function, is not proven. However, we prove a shift relation in section 4 for a generalization of the braiding (3.42). This relation expresses in particular the braiding for a given $\widehat{V}_{-K b h_{1}}$ in terms of that for $\widehat{V}_{-(K-1) b h_{1}}$ and the braiding and fusion for $\widehat{V}_{-b h_{1}}$. By induction on $K$ this proves that the braiding matrix given here is correct.

\section{Braiding kernel}

Section 4.1 conjectures the braiding kernel (4.4) of two semi-degenerate vertex operators, which generalizes the braiding/fusion kernel for Virasoro $(N=2)$ conformal blocks $[44,45]$. We explain in section 4.2 how it reduces to the braiding matrix (3.42) and in section 4.3 that it obeys a very constraining shift relation deduced from a Moore-Seiberg pentagon identity. Discrete symmetries are investigated later in section 5.3.

\subsection{Main formula}

The four-point function with two generic momenta $\alpha_{1}, \alpha_{3}$ and two semi-degenerate momenta $\alpha_{2}=\kappa_{2} h_{1}, \alpha_{4}=\kappa_{4} h_{1}$ has an s-channel decomposition

$$
\begin{aligned}
& \left\langle\widehat{V}_{\alpha_{3}}(\infty) \widehat{V}_{\kappa_{4} h_{1}}(1) \widehat{V}_{\kappa_{2} h_{1}}(x, \bar{x}) \widehat{V}_{\alpha_{1}}(0)\right\rangle
\end{aligned}
$$

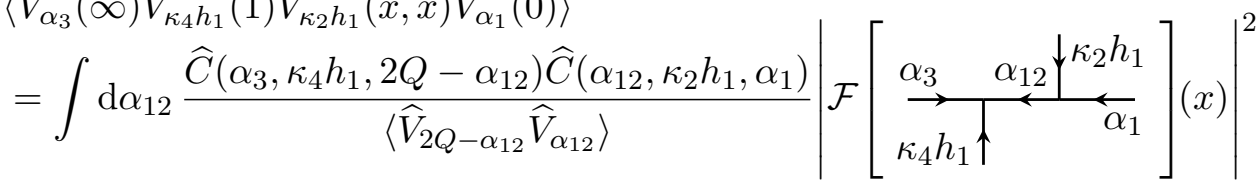

where the denominator is non-zero because of our Weyl-invariant choice of normalization of vertex operators, and $|\cdots|^{2}$ involves conjugating $x$ but not momenta. Note that the internal momentum $\alpha_{12}$ is continuous rather than discrete because there is no fully degenerate vertex operator. The s-channel conformal blocks are in principle fixed by $W_{N}$ symmetry. In practice, closed forms are only known thanks to the AGT relation with instanton partition functions, and we will not need them. In this section we again normalize conformal blocks as $\mathcal{F}_{\alpha_{12}}^{(\mathrm{s})}(x)=(-x)^{\Delta\left(\alpha_{12}\right)-\Delta\left(\alpha_{1}\right)-\Delta\left(\kappa_{2} h_{1}\right)}(1+\cdots)$ : the use of $-x$ instead of $x$ avoids phases.

The u-channel counterpart of (4.1) has $\kappa_{2} \leftrightarrow \kappa_{4}$ :

$$
\begin{aligned}
& \left\langle\widehat{V}_{\alpha_{3}}(\infty) \widehat{V}_{\kappa_{4} h_{1}}(1) \widehat{V}_{\kappa_{2} h_{1}}(x, \bar{x}) \widehat{V}_{\alpha_{1}}(0)\right\rangle \\
& =\int \mathrm{d} \alpha_{32} \frac{\widehat{C}\left(\alpha_{3}, \kappa_{2} h_{1}, \alpha_{32}\right) \widehat{C}\left(2 Q-\alpha_{32}, \kappa_{4} h_{1}, \alpha_{1}\right)}{\left\langle\widehat{V}_{2 Q-\alpha_{32}} \widehat{V}_{\alpha_{32}}\right\rangle}\left|\mathcal{F}\left[\underset{\alpha_{3} \alpha_{32} \uparrow_{\kappa_{4} h_{1}}^{\kappa_{2} h_{1} \downarrow}}{\stackrel{\alpha_{1}}{\longleftarrow}}\right](x)\right|^{2} .
\end{aligned}
$$


Again, we normalize these $\mathrm{u}$-channel conformal blocks so that their leading term is a power of $(-x)$, namely $\mathcal{F}_{\alpha_{32}}^{(\mathrm{u})}(x) \sim(-x)^{\Delta\left(\alpha_{3}\right)-\Delta\left(\alpha_{32}\right)-\Delta\left(\kappa_{2} h_{1}\right)}$. Both sets of conformal blocks are analytic on $\mathbb{C} \backslash[0, \infty)$. The two decompositions are related by an integral transformation

$$
\mathcal{F}_{\alpha_{12}}^{(\mathrm{s})}(x)=\int \mathrm{d} \alpha_{32} \mathbf{B}_{\alpha_{12} \alpha_{32}} \mathcal{F}_{\alpha_{32}}^{(\mathrm{u})}(x)
$$

Our goal is to find the braiding kernel $\mathbf{B}_{\alpha_{12} \alpha_{32}}$.

From section 3.2 we know this braiding kernel in the limit $\kappa_{2} h_{1} \rightarrow-K b h_{1}$, in other words when one of the semi-degenerate operators turns into a degenerate operator. Then it is a sum of residues (hence an integral) of a product of sines (3.42) which involve various multiples of $b^{2}$ in their arguments. This product of sines can be recast in terms of the double Sine function $S_{b}$ which obeys $S_{b}(x+n b) / S_{b}(x)=\prod_{\mu=0}^{n-1} 2 \sin \pi\left(b x+\mu b^{2}\right)$ (see section 2.3 on special functions). The braiding kernel for generic $\kappa_{2}$ should thus be an integral of some $S_{b}$ functions. Writing all generic momenta as $\alpha=Q-\mathrm{i} a$, we propose

$$
\begin{aligned}
& \mathbf{B}_{\alpha_{12} \alpha_{32}}\left[\begin{array}{cc}
\kappa_{4} h_{1} & \kappa_{2} h_{1} \\
\alpha_{3} & \alpha_{1}
\end{array}\right]=\mathrm{i}^{N-1} \prod_{s \neq t}^{N}\left[\frac{\Gamma_{b}\left(b+b^{-1}+\left\langle\mathrm{i} a_{12}, h_{s}-h_{t}\right\rangle\right)}{\Gamma_{b}\left(\left\langle\mathrm{i} a_{32}, h_{s}-h_{t}\right\rangle\right)}\right] \\
& \times \prod_{s, t=1}^{N}\left[\frac{\Gamma_{b}\left(\frac{\kappa_{2}}{N}+\left\langle\mathrm{i} a_{3}, h_{s}\right\rangle-\left\langle\mathrm{i} a_{32}, h_{t}\right\rangle\right) \Gamma_{b}\left(b+b^{-1}-\frac{\kappa_{4}}{N}-\left\langle\mathrm{i} a_{1}, h_{s}\right\rangle-\left\langle\mathrm{i} a_{32}, h_{t}\right\rangle\right)}{\Gamma_{b}\left(\frac{\kappa_{2}}{N}+\left\langle\mathrm{i} a_{1}, h_{s}\right\rangle-\left\langle\mathrm{i} a_{12}, h_{t}\right\rangle\right) \Gamma_{b}\left(b+b^{-1}-\frac{\kappa_{4}}{N}-\left\langle\mathrm{i} a_{3}, h_{s}\right\rangle-\left\langle\mathrm{i} a_{12}, h_{t}\right\rangle\right)}\right] \\
& \times \int \frac{\mathrm{d}^{N-1} \tau}{\prod_{i \neq j}^{N-1} S_{b}\left(\mathrm{i} \tau_{i}-\mathrm{i} \tau_{j}\right)} \prod_{j=1}^{N-1} \prod_{s=1}^{N}\left[\frac{S_{b}\left(-\left\langle\mathrm{i} a_{3}, h_{s}\right\rangle+\mathrm{i} \tau_{j}\right) S_{b}\left(\frac{\kappa_{2}+\kappa_{4}}{N}-b-b^{-1}+\left\langle\mathrm{i} a_{1}, h_{s}\right\rangle+\mathrm{i} \tau_{j}\right)}{S_{b}\left(\frac{\kappa_{2}}{N}-\left\langle\mathrm{i} a_{32}, h_{s}\right\rangle+\mathrm{i} \tau_{j}\right) S_{b}\left(\frac{\kappa_{4}}{N}+\left\langle\mathrm{i} a_{12}, h_{s}\right\rangle+\mathrm{i} \tau_{j}\right)}\right]
\end{aligned}
$$

up to a constant factor that does not depend on any momentum. The integration contours go from $-\infty$ to $\infty$ with poles of the numerator $S_{b}$ functions above the contours, and zeros of the denominator below them. For instance, if all components $\left\langle\mathrm{i} a_{1}, h_{s}\right\rangle,\left\langle\mathrm{i} a_{3}, h_{2}\right\rangle,\left\langle\mathrm{i} a_{12}, h_{2}\right\rangle$, $\left\langle\mathrm{i} a_{32}, h_{2}\right\rangle$ are purely imaginary and all $\operatorname{Re} \frac{\kappa_{i}}{N}=\left(b+b^{-1}\right) / 2$ then contours can be taken to be horizontal lines with $-\left(b+b^{-1}\right) / 2<\operatorname{Im}\left(\tau_{j}\right)<0$. For other values of momenta, the contour is deformed to keep the same set of poles on each side. Another remark is that $\prod_{i \neq j}^{N-1} 1 / S_{b}\left(\mathrm{i} \tau_{i}-\mathrm{i} \tau_{j}\right)$ has no pole: it simplifies to a product of sines (4.13).

In a normalization of conformal blocks where the leading term is a power of $x$, the braiding kernel includes phases, depending on the $\operatorname{sign} \epsilon$ of $\operatorname{Im} x$ :

$$
\mathbf{B}_{\alpha_{12} \alpha_{32}}^{\epsilon}\left[\begin{array}{cc}
\kappa_{4} h_{1} & \kappa_{2} h_{1} \\
\alpha_{3} & \alpha_{1}
\end{array}\right]=e^{\mathrm{i} \pi \epsilon\left[\Delta\left(\alpha_{12}\right)-\Delta\left(\alpha_{1}\right)+\Delta\left(\alpha_{32}\right)-\Delta\left(\alpha_{3}\right)\right]} \mathbf{B}_{\alpha_{12} \alpha_{32}}\left[\begin{array}{cc}
\kappa_{4} h_{1} & \kappa_{2} h_{1} \\
\alpha_{3} & \alpha_{1}
\end{array}\right]
$$

A preliminary check of (4.4) is that it reproduces known results [44] for the Liouville theory $(N=2)$. In their equation (48) replace their $Q$ by $b+b^{-1}$, shift the integration variable $s \rightarrow s-\alpha_{21}+\alpha_{4}-\left(b+b^{-1}\right) / 2$, then map $\alpha_{2} \rightarrow b+b^{-1}-\alpha_{2}$ (for $N=2$ this is a Weyl symmetry). The factors with $U_{3,4}$ become $S_{b}\left( \pm\left(\alpha_{3}-\left(b+b^{-1}\right) / 2\right)+s\right)$. The factors with $U_{1,2}$ become $S_{b}\left(\alpha_{4}+\alpha_{2}-b-b^{-1} \pm\left(\alpha_{1}-\left(b+b^{-1}\right) / 2\right)+s\right)$. The denominator factors with $\widehat{V}_{1,2}$ become $S_{b}\left(\alpha_{2} \pm\left(\alpha_{32}-\left(b+b^{-1}\right) / 2\right)+s\right)$. The denominator factors with $\widehat{V}_{3,4}$ become $S_{b}\left(\alpha_{4} \pm\left(\alpha_{21}-\left(b+b^{-1}\right) / 2\right)+s\right)$. Thus, the integrand from [44, 45] coincides with that of (4.4) for $N=2$. It is straightforward to check that prefactors also coincide. 


\subsection{Reduction to fully degenerate}

We now describe how to take the limit $\kappa_{2} h_{1} \rightarrow-K b h_{1}$ in (4.4), and retrieve the sum of residues from section 3.2 .

The integrand in (4.4) has poles at

$$
\mathrm{i} \tau_{j}=\left\{\begin{array}{l}
\left\langle\mathrm{i} a_{3}, h_{s}\right\rangle-m b-n / b \\
b+b^{-1}-\frac{\kappa_{2}}{N}-\frac{\kappa_{4}}{N}-\left\langle\mathrm{i} a_{1}, h_{s}\right\rangle-m b-n / b
\end{array}\right.
$$

and

$$
\mathrm{i} \tau_{j}=\left\{\begin{array}{l}
b+b^{-1}-\frac{\kappa_{2}}{N}+\left\langle\mathrm{i} a_{32}, h_{s}\right\rangle+m b+n / b \\
b+b^{-1}-\frac{\kappa_{4}}{N}-\left\langle\mathrm{i} a_{12}, h_{s}\right\rangle+m b+n / b
\end{array}\right.
$$

for integers $m, n \geq 0$. As mentionned before, the contour for $\mathrm{i} \tau$ is chosen with poles (4.6) on the left and poles (4.7) on the right. This is possible as long as the two sets of poles are disjoint. Otherwise, the contour is pinched between the two sets and the integral diverges.

To understand the divergence, consider a simple model of a contour integral pinched by poles getting close together from the two sides of the contour. Let $f(z)$ be holomorphic in a neighborhood of $a$, and $a_{L}$ and $a_{R}$ be points in this neighborhood. Then

$$
\int_{\text {between }} \mathrm{d} z \frac{f(z)}{\left(z-a_{L}\right)\left(z-a_{R}\right)}=2 \pi \mathrm{i} \frac{f\left(a_{L}\right)}{a_{L}-a_{R}}+\int_{\text {left }} \mathrm{d} z \frac{f(z)}{\left(z-a_{L}\right)\left(z-a_{R}\right)}
$$

where the initial contour goes between the two points, with $a_{L}$ on its left and $a_{R}$ on its right, and where the second contour is moved through $a_{L}$. As $a_{L}, a_{R} \rightarrow a$, the second term is regular, so the residue is $2 \pi \mathrm{i} f(a)$. This residue is obtained from the original integrand by taking the limit $a_{L}, a_{R} \rightarrow a$ then considering the second residue of the result $f(z) /(z-a)^{2}$. We denote this operation of taking the second residue as res $^{2}$.

In our case there are two rather different pinchings by poles (4.6) and (4.7). Whenever one of the (4.6) is equal to $b+b^{-1}-\frac{\kappa_{2}}{N}+\left\langle\mathrm{i} a_{32}, h_{s}\right\rangle+m b+n / b$ the braiding kernel is singular. In fact, these singularities, together with those of numerator $\Gamma_{b}$ functions in (4.4), precisely reproduce singularities of the $\mathrm{u}$-channel Toda CFT three-point functions:

$$
\begin{aligned}
& \widehat{C}\left(\alpha_{3}, \kappa_{2} h_{1}, 2 Q-\alpha_{32}\right) \widehat{C}\left(\alpha_{32}, \kappa_{4} h_{1}, \alpha_{1}\right) \\
& =\frac{1}{\prod_{t, u}\left[\Upsilon_{b}\left(\frac{\kappa_{2}}{N}+\left\langle\mathrm{i} a_{3}, h_{t}\right\rangle-\left\langle\mathrm{i} a_{32}, h_{u}\right\rangle\right) \Upsilon_{b}\left(\frac{\kappa_{4}}{N}+\left\langle\mathrm{i} a_{1}, h_{t}\right\rangle+\left\langle\mathrm{i} a_{32}, h_{u}\right\rangle\right)\right]} .
\end{aligned}
$$

It may be interesting to pursue further the analysis by considering multiple singularities, keeping in mind the constraints $\sum_{t}\left\langle\mathrm{i} a_{j}, h_{t}\right\rangle=0$ for each momentum. On the other hand, if one of the (4.6) is equal to $b+b^{-1}-\frac{\kappa_{4}}{N}-\left\langle\mathrm{i} a_{12}, h_{s}\right\rangle+m b+n / b$, the contour is also pinched, but the prefactors in (4.4) (specifically the denominator $\Gamma_{b}$ functions) cancel the singularity so the braiding kernel can have a finite limit.

We are ready to consider our limit of interest: $\kappa_{2}=-K b+N$ i $\varepsilon$ for $\varepsilon \rightarrow 0$ (and $\varepsilon>0$ ). The OPE of $\widehat{V}_{-K b h_{1}}$ with a generic vertex operator constrains $\alpha_{12}$ and $\alpha_{32}$, so we further focus on $\alpha_{12}=\alpha_{1}-b h_{[n]}$ and $\alpha_{32}=\alpha_{3}-b h_{[\tilde{n}]}$ for some weights $h_{[n]}$ and $h_{[\tilde{n}]}$ in $\mathcal{R}\left(K h_{1}\right)$. 
To simplify some expressions we write $\kappa_{4}=\varkappa+K b$. We keep $\alpha_{1}$ and $\alpha_{3}$ generic. The poles (4.6) and (4.7) are now respectively at

$$
\begin{aligned}
& \mathrm{i} \tau_{j}=\left\{\begin{array}{l}
\left\langle\mathrm{i} a_{3}, h_{s}\right\rangle-m b-n / b, \\
b+b^{-1}-\frac{\varkappa}{N}-\left\langle\mathrm{i} a_{1}, h_{s}\right\rangle-m b-n / b-\mathrm{i} \varepsilon,
\end{array}\right. \\
& \mathrm{i} \tau_{j}=\left\{\begin{array}{l}
b+b^{-1}+\left\langle\mathrm{i} a_{3}, h_{s}\right\rangle+b \widetilde{n}_{s}+m b+n / b-\mathrm{i} \varepsilon, \\
b+b^{-1}-\frac{\varkappa}{N}-\left\langle\mathrm{i} a_{1}, h_{s}\right\rangle-b n_{s}+m b+n / b .
\end{array}\right.
\end{aligned}
$$

Because the momenta $\alpha_{1}$ and $\alpha_{3}$ are generic, the contour is pinched as $\varepsilon \rightarrow 0$ precisely when $\mathrm{i} \tau_{j}=b+b^{-1}-\frac{\varkappa}{N}-\left\langle\mathrm{i} a_{1}, h_{s}\right\rangle-b l$ for any $1 \leq j \leq N-1,1 \leq s \leq N$ and $0 \leq l \leq n_{s}$. The most singular contribution to the integral, of order $1 / \varepsilon^{N-1}$, comes from values of $\mathrm{i} \tau$ where all $\mathrm{i} \tau_{j}$ take this form.

We only describe the contour integral part of the braiding matrix (4.4), as prefactors only lengthen computations. The coefficient of the term of order $1 / \varepsilon^{N-1}$ in this integral is

$$
\begin{aligned}
I= & \prod_{j=1}^{N-1}\left[\sum_{p_{j}=1}^{N} \sum_{k_{j}=0}^{n_{p_{j}}} \begin{array}{c}
\mathrm{res}_{j}^{2}=b+b^{-1}-\frac{\varkappa}{N}-\left\langle\mathrm{ii}_{1}, h_{p_{j}}\right\rangle-k_{j} b
\end{array}\right]\left\{\prod_{i \neq j}^{N-1} \frac{1}{S_{b}\left(\mathrm{i} \tau_{i}-\mathrm{i} \tau_{j}\right)}\right. \\
& \left.\times \prod_{j=1}^{N-1} \prod_{s=1}^{N}\left[\frac{S_{b}\left(-\left\langle\mathrm{i} a_{3}, h_{s}\right\rangle+\mathrm{i} \tau_{j}\right)}{S_{b}\left(-\left\langle\mathrm{i} a_{3}, h_{s}\right\rangle-\widetilde{n}_{s} b+\mathrm{i} \tau_{j}\right)} \frac{S_{b}\left(\frac{\varkappa}{N}-b-b^{-1}+\left\langle\mathrm{i} a_{1}, h_{s}\right\rangle+\mathrm{i} \tau_{j}\right)}{S_{b}\left(\frac{\varkappa}{N}+\left\langle\mathrm{i} a_{1}, h_{s}\right\rangle+n_{s} b+\mathrm{i} \tau_{j}\right)}\right]\right\} .
\end{aligned}
$$

Note that

$$
\prod_{i \neq j}^{N-1} \frac{1}{S_{b}\left(\mathrm{i} \tau_{i}-\mathrm{i} \tau_{j}\right)}=\prod_{i<j}^{N-1}\left(-4 \sin \pi b\left(\mathrm{i} \tau_{i}-\mathrm{i} \tau_{j}\right) \sin \frac{\pi}{b}\left(\mathrm{i} \tau_{i}-\mathrm{i} \tau_{j}\right)\right) .
$$

The shift relations for $\Gamma_{b}$ and $S_{b}$ yield

$$
\begin{aligned}
I= & \prod_{j=1}^{N-1}\left[\sum_{p_{j}=1}^{N} \sum_{k_{j}=0}^{n_{p_{j}}} \begin{array}{c}
\mathrm{i}_{j} \tau_{j}=b+b^{-1}-\frac{\varkappa}{N}-\left\langle\mathrm{ia}_{1}, h_{p_{j}}\right\rangle-k_{j} b
\end{array}\right]\{ \\
& \frac{\prod_{i<j}^{N-1}\left[-4 b^{2} \sin \pi b\left(\mathrm{i} \tau_{i}-\mathrm{i} \tau_{j}\right) \sin \frac{\pi}{b}\left(\mathrm{i} \tau_{i}-\mathrm{i} \tau_{j}\right)\right]}{\prod_{j=1}^{N-1} \prod_{s=1}^{N}\left[2 \sin \frac{\pi}{b}\left(\frac{\varkappa}{N}-b-b^{-1}+\left\langle\mathrm{i} a_{1}, h_{s}\right\rangle+\mathrm{i} \tau_{j}\right)\right]} \\
& \left.\times \prod_{j=1}^{N-1} \prod_{s=1}^{N}\left[\frac{\prod_{\mu=0}^{\widetilde{n}_{s}-1}\left[2 \sin \pi\left(-b^{2}-\mu b^{2}-b\left\langle\mathrm{i} a_{3}, h_{s}\right\rangle+b \mathrm{i} \tau_{j}\right)\right]}{\prod_{\mu=0}^{n_{s}}\left[2 \sin \pi\left(-b^{2}+\mu b^{2}+\frac{b \varkappa}{N}+b\left\langle\mathrm{i} a_{1}, h_{s}\right\rangle+b \mathrm{i} \tau_{j}\right)\right]}\right]\right\} .
\end{aligned}
$$

This expression differs from the desired sum of residues (3.42) in the following respects: a redefinition $\mathrm{i} \tau_{j} \rightarrow b \mathrm{i} \tau_{j}+\frac{b \varkappa}{N}-b^{2}-1$, a sum over choices of the $p_{j}$, and additional factors of the form $\sin \frac{\pi}{b}(\cdots)$. Because of antiperiodicity of sine these factors are independent of the $k_{j}$ except for a sign. After extracting a sign, these factors are

$$
\frac{\prod_{i<j}^{N-1} \sin \frac{\pi}{b}\left(\left\langle\mathrm{i} a_{1}, h_{p_{i}}-h_{p_{j}}\right\rangle\right)}{\prod_{j=1}^{N-1} \prod_{s \neq p_{j}}^{N} \sin \frac{\pi}{b}\left(\left\langle\mathrm{i} a_{1}, h_{s}-h_{p_{j}}\right\rangle\right)}=\left\{\begin{array}{l}
0 \quad \text { if two } p_{i} \text { coincide, otherwise } \\
1 / \prod_{j=1}^{N-1} \prod_{i=j+1}^{N} \sin \frac{\pi}{b}\left(\left\langle\mathrm{i} a_{1}, h_{p_{i}}-h_{p_{j}}\right\rangle\right)
\end{array}\right.
$$


where $p_{N}$ denotes the (single) element of $\llbracket 1, N \rrbracket \backslash\left\{p_{i} \mid i<N\right\}$ so that $p$ is a permutation of $\llbracket 1, N \rrbracket$. Then these factors are independent of the permutation $p$, except for a sign: the signature of $p$. For each permutation $p$ we get a sum of residues times the signature of $p$, and this structure coincides with that of (3.42). Below that equation we had proven that it is independent of the permutation, hence summing over permutation simply introduces a trivial factor. We have thus reproduced qualitatively the structure of the braiding matrix of $\widehat{V}_{-K b h_{1}}$ by taking the appropriate limit of the braiding kernel. The exact reduction of the braiding kernel to the braiding matrix is confirmed by a more detailed calculation.

\subsection{Shift relation from pentagon identity}

Braiding and fusion kernels (or matrices) obey Moore-Seiberg pentagon and hexagon relations. Here we consider a particular pentagon relation shown in figure 1. Going through the moves $1 \rightarrow 2 \rightarrow 3$ we find

$$
\begin{aligned}
\mathcal{F}[1] & =\int \mathrm{d} \alpha_{32} \mathbf{B}_{\alpha_{12} \alpha_{32}}\left[\begin{array}{cc}
\kappa_{4} h_{1} & \kappa_{2} h_{1} \\
\alpha_{3} & \alpha_{1}
\end{array}\right] \mathcal{F}[2] \\
& =\int \mathrm{d} \alpha_{32} \sum_{s=1}^{N} \mathbf{B}_{\alpha_{12} \alpha_{32}}\left[\begin{array}{cc}
\kappa_{4} h_{1} & \kappa_{2} h_{1} \\
\alpha_{3} & \alpha_{1}
\end{array}\right] \mathbf{F}_{s}\left[\begin{array}{cc}
\left(\kappa_{2}+b\right) h_{1} & -b h_{1} \\
\alpha_{3} & 2 Q-\alpha_{32}
\end{array}\right] \mathcal{F}[3]
\end{aligned}
$$

On the other hand, going through the moves $1 \rightarrow 4 \rightarrow 5 \rightarrow 3$ yields

$$
\begin{aligned}
& \mathcal{F}[1]=\sum_{p=1}^{N} \mathbf{F}_{p}\left[\begin{array}{cc}
\left(\kappa_{2}+b\right) h_{1} & -b h_{1} \\
2 Q-\alpha_{12} & \alpha_{1}
\end{array}\right] \mathcal{F}[4] \\
& =\sum_{p=1}^{N} \int \mathrm{d} \alpha_{32}^{\prime} \mathbf{F}_{p}\left[\begin{array}{cc}
\left(\kappa_{2}+b\right) h_{1} & -b h_{1} \\
2 Q-\alpha_{12} & \alpha_{1}
\end{array}\right] \mathbf{B}_{\alpha_{12} \alpha_{32}^{\prime}}\left[\begin{array}{cc}
\kappa_{4} h_{1} & \left(\kappa_{2}+b\right) h_{1} \\
\alpha_{3} & \alpha_{1}-b h_{p}
\end{array}\right] \mathcal{F}[5] \\
& =\sum_{p, s=1}^{N} \int \mathrm{d} \alpha_{32}^{\prime} \mathbf{F}_{p}\left[\begin{array}{cc}
\left(\kappa_{2}+b\right) h_{1} & -b h_{1} \\
2 Q-\alpha_{12} & \alpha_{1}
\end{array}\right] \mathbf{B}_{\alpha_{12} \alpha_{32}^{\prime}}\left[\begin{array}{cc}
\kappa_{4} h_{1} & \left(\kappa_{2}+b\right) h_{1} \\
\alpha_{3} & \alpha_{1}-b h_{p}
\end{array}\right] \mathbf{B}_{p s}\left[\begin{array}{cc}
\kappa_{4} h_{1} & -b h_{1} \\
\alpha_{32}^{\prime} & \alpha_{1}
\end{array}\right] \mathcal{F}[3] .
\end{aligned}
$$

The coefficients of each conformal block $\mathcal{F}[3]$ (these are labelled by the choice of $1 \leq s \leq N$ and $\alpha_{32}=\alpha_{32}^{\prime}-b h_{s}$ ) must be the same in (4.17) and (4.20).

To check that the proposal (4.4) obeys the pentagon identity, we will need the braiding matrix obtained from (3.24) using $\alpha_{1}=Q-\mathrm{i} a_{1}$ and $\alpha_{32}^{\prime}=Q-\mathrm{i} a_{32}+b h_{s}$ :

$$
\begin{aligned}
& \mathbf{B}_{p s}\left[\begin{array}{cc}
\kappa_{4} h_{1} & -b h_{1} \\
\alpha_{32}^{\prime} & \alpha_{1}
\end{array}\right] \\
& =\prod_{t \neq p}^{N} \frac{\Gamma\left(1+b\left\langle\mathrm{i} a_{1}, h_{t}-h_{p}\right\rangle\right)}{\Gamma\left(\frac{b \kappa_{4}}{N}+b\left\langle\mathrm{i} a_{1}, h_{t}\right\rangle+b\left\langle\mathrm{i} a_{32}, h_{s}\right\rangle-b^{2}\right)} \prod_{u \neq s}^{N} \frac{\Gamma\left(b\left\langle\mathrm{i} a_{32}, h_{s}-h_{u}\right\rangle-b^{2}\right)}{\Gamma\left(1-\frac{b \kappa_{4}}{N}-b\left\langle\mathrm{i} a_{1}, h_{p}\right\rangle-b\left\langle\mathrm{i} a_{32}, h_{u}\right\rangle\right)} .
\end{aligned}
$$




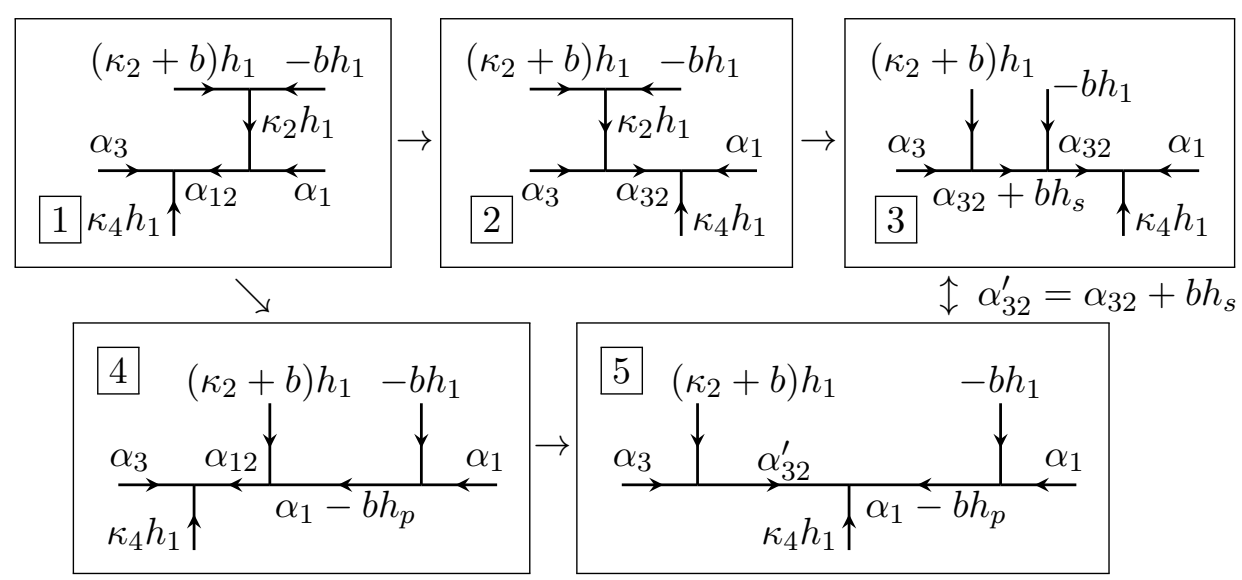

Figure 1. Pentagon identity. $1 \rightarrow 2$ and $4 \rightarrow 5$ are braidings of two semi-degenerates, $2 \rightarrow 3$ and $1 \rightarrow 4$ are known fusions of $\widehat{V}_{-b h_{1}}$ and a semi-degenerate, $5 \leftrightarrow 3$ is a known braiding of $\widehat{V}_{-b h_{1}}$ and a semi-degenerate.

We will also need coefficients of the fusion of $\left(\kappa_{2}+b\right) h_{1}$ and $-b h_{1}$ into $\kappa_{2} h_{1}$, which can be deduced from the braiding matrix (3.24), as done in equation (B.14) of the reference [56].

$$
\mathbf{F}_{p}\left[\begin{array}{cc}
\left(\kappa_{2}+b\right) h_{1} & -b h_{1} \\
Q-\mathrm{i} a^{\prime} & Q-\mathrm{i} a
\end{array}\right]=\Gamma\left(b \kappa_{2}\right) \frac{\prod_{t \neq p}^{N} \Gamma\left(b\left\langle\mathrm{i} a, h_{p}-h_{t}\right\rangle\right)}{\prod_{t=1}^{N} \Gamma\left(\frac{b \kappa_{2}}{N}+b\left\langle\mathrm{i} a, h_{p}\right\rangle+b\left\langle\mathrm{i} a^{\prime}, h_{t}\right\rangle\right)} .
$$

We now write down (4.20) explicitly for a fixed choice of $\alpha_{32}$ and of $1 \leq s \leq N$, and simplify it in order to find (4.17). All generic momenta are written as $\alpha=Q-\mathrm{i} a$ and we denote $\mathrm{i} a^{u}=\left\langle\mathrm{i} a, h_{u}\right\rangle$ for conciseness. Note that $\alpha_{32}^{\prime}=Q-\mathrm{i} a_{32}+b h_{s}$.

Let us start!

$$
\begin{aligned}
& \sum_{p=1}^{N} \mathbf{F}_{p}\left[\begin{array}{cc}
\left(\kappa_{2}+b\right) h_{1} & -b h_{1} \\
2 Q-\alpha_{12} & \alpha_{1}
\end{array}\right] \mathbf{B}_{\alpha_{12} \alpha_{32}^{\prime}}\left[\begin{array}{cc}
\kappa_{4} h_{1} & \left(\kappa_{2}+b\right) h_{1} \\
\alpha_{3} & \alpha_{1}-b h_{p}
\end{array}\right] \mathbf{B}_{p s}\left[\begin{array}{cc}
\kappa_{4} h_{1} & -b h_{1} \\
\alpha_{32}^{\prime} & \alpha_{1}
\end{array}\right] \\
& =\sum_{p=1}^{N}\left(\Gamma\left(b \kappa_{2}\right) \frac{\prod_{t \neq p}^{N} \Gamma\left(b \mathrm{i} a_{1}^{p}-b \mathrm{i} a_{1}^{t}\right)}{\prod_{t=1}^{N} \Gamma\left(\frac{b \kappa_{2}}{N}+b \mathrm{i} a_{1}^{p}-b \mathrm{i} a_{12}^{t}\right)} \prod_{t \neq u}^{N} \frac{\Gamma_{b}\left(b+b^{-1}+\mathrm{i} a_{12}^{t}-\mathrm{i} a_{12}^{u}\right)}{\Gamma_{b}\left(\mathrm{i} a_{32}^{t}-\mathrm{i} a_{32}^{u}-b \delta_{s, t}+b \delta_{s, u}\right)}\right. \\
& \times \prod_{t, u=1}^{N}\left[\frac{\Gamma_{b}\left(\frac{\kappa_{2}}{N}+\mathrm{i} a_{3}^{t}-\mathrm{i} a_{32}^{u}+b \delta_{s, u}\right) \Gamma_{b}\left(b+b^{-1}-\frac{\kappa_{4}}{N}-\mathrm{i} a_{1}^{t}-\mathrm{i} a_{32}^{u}-b \delta_{p, t}+b \delta_{s, u}\right)}{\Gamma_{b}\left(\frac{\kappa_{2}}{N}+\mathrm{i} a_{1}^{t}-\mathrm{i} a_{12}^{u}+b \delta_{p, t}\right) \Gamma_{b}\left(b+b^{-1}-\frac{\kappa_{4}}{N}-\mathrm{i} a_{3}^{t}-\mathrm{i} a_{12}^{u}\right)}\right] \\
& \times \int \frac{\mathrm{d}^{N-1} \tau_{j}}{\prod_{i \neq j}^{N-1} S_{b}\left(\mathrm{i} \tau_{i}-\mathrm{i} \tau_{j}\right)} \prod_{j=1}^{N-1} \prod_{t=1}^{N} \frac{S_{b}\left(-\mathrm{i} a_{3}^{t}+\mathrm{i} \tau_{j}\right) S_{b}\left(\frac{\kappa_{2}}{N}+\frac{\kappa_{4}}{N}-b-b^{-1}+\mathrm{i} a_{1}^{t}+\mathrm{i} \tau_{j}+b \delta_{p, t}\right)}{S_{b}\left(\frac{\kappa_{2}}{N}-\mathrm{i} a_{32}^{t}+\mathrm{i} \tau_{j}+b \delta_{s, t}\right) S_{b}\left(\frac{\kappa_{4}}{N}+\mathrm{i} a_{12}^{t}+\mathrm{i} \tau_{j}\right)} \\
& \left.\times \prod_{t \neq p}^{N} \frac{\Gamma\left(1+b \mathrm{i} a_{1}^{t}-b \mathrm{i} a_{1}^{p}\right)}{\Gamma\left(\frac{b \kappa_{4}}{N}+b \mathrm{i} a_{1}^{t}+b \mathrm{i} a_{32}^{s}-b^{2}\right)} \prod_{u \neq s}^{N} \frac{\Gamma\left(b \mathrm{i} a_{32}^{s}-b \mathrm{i} a_{32}^{u}-b^{2}\right)}{\Gamma\left(1-\frac{b \kappa_{4}}{N}-b \mathrm{i} a_{1}^{p}-b \mathrm{i} a_{32}^{u}\right)}\right) .
\end{aligned}
$$


We collect factors which do not depend on $p, s$ using shift relations of $\Gamma_{b}$ and $S_{b}$. Factors of $\sqrt{2 \pi}$ and powers of $b$ cancel, and we combine many Gamma as $\Gamma(x) \Gamma(1-x)=\pi / \sin \pi x$.

$$
\begin{aligned}
=\prod_{t, u=1}^{N}\left[\frac{\Gamma_{b}\left(\frac{\kappa_{2}}{N}+\mathrm{i} a_{3}^{t}-\mathrm{i} a_{32}^{u}\right) \Gamma_{b}\left(b+b^{-1}-\frac{\kappa_{4}}{N}-\mathrm{i} a_{1}^{t}-\mathrm{i} a_{32}^{u}\right)}{\Gamma_{b}\left(\frac{\kappa_{2}}{N}+\mathrm{i} a_{1}^{t}-\mathrm{i} a_{12}^{u}\right) \Gamma_{b}\left(b+b^{-1}-\frac{\kappa_{4}}{N}-\mathrm{i} a_{3}^{t}-\mathrm{i} a_{12}^{u}\right)}\right] \prod_{t \neq u}^{N}\left[\frac{\Gamma_{b}\left(b+b^{-1}+\mathrm{i} a_{12}^{t}-\mathrm{i} a_{12}^{u}\right)}{\Gamma_{b}\left(\mathrm{i} a_{32}^{t}-\mathrm{i} a_{32}^{u}\right)}\right] \\
\times \int \frac{\mathrm{d}^{N-1} \tau_{j}}{\prod_{i \neq j}^{N-1} S_{b}\left(\mathrm{i} \tau_{i}-\mathrm{i} \tau_{j}\right)}\left\{\prod_{j=1}^{N-1} \prod_{t=1}^{N}\left[\frac{S_{b}\left(-\mathrm{i} a_{3}^{t}+\mathrm{i} \tau_{j}\right) S_{b}\left(\frac{\kappa_{2}}{N}+\frac{\kappa_{4}}{N}-b-b^{-1}+\mathrm{i} a_{1}^{t}+\mathrm{i} \tau_{j}\right)}{S_{b}\left(\frac{\kappa_{2}}{N}-\mathrm{i} a_{32}^{t}+\mathrm{i} \tau_{j}\right) S_{b}\left(\frac{\kappa_{4}}{N}+\mathrm{i} a_{12}^{t}+\mathrm{i} \tau_{j}\right)}\right]\right. \\
\quad \times \frac{\Gamma\left(b \kappa_{2}\right) \prod_{u \neq s}^{N} \Gamma\left(b \mathrm{i} a_{32}^{u}-b \mathrm{i} a_{32}^{s}\right) \prod_{t=1}^{N} \frac{1}{\pi} \sin \pi\left(\frac{b \kappa_{4}}{N}+b \mathrm{i} a_{1}^{t}+b \mathrm{i} a_{32}^{s}-b^{2}\right)}{\prod_{j=1}^{N-1} \frac{1}{\pi} \sin \pi b\left(\frac{\kappa_{2}}{N}-\mathrm{i} a_{32}^{s}+\mathrm{i} \tau_{j}\right) \prod_{t=1}^{N} \Gamma\left(\frac{b \kappa_{2}}{N}+b \mathrm{i} a_{3}^{t}-b \mathrm{i} a_{32}^{s}\right)} \\
\left.\quad \times \sum_{p=1}^{N} \frac{\prod_{j=1}^{N-1} \frac{1}{\pi} \sin \pi b\left(\frac{\kappa_{2}}{N}+\frac{\kappa_{4}}{N}-b-b^{-1}+\mathrm{i} a_{1}^{p}+\mathrm{i} \tau_{j}\right)}{\sin \pi\left(\frac{b \kappa_{4}}{N}+b \mathrm{i} a_{1}^{p}+b \mathrm{i} a_{32}^{s}-b^{2}\right) \prod_{t \neq p}^{N} \frac{1}{\pi} \sin \pi\left(b \mathrm{i} a_{1}^{p}-b \mathrm{i} a_{1}^{t}\right)}\right\}
\end{aligned}
$$

The last line is a sum of residues at $v=b \mathrm{i} a_{1}^{p}$ of $\prod_{j=1}^{N-1}\left[\frac{1}{\pi} \sin \pi\left(\frac{b \kappa_{2}}{N}+\frac{b \kappa_{4}}{N}-b^{2}-1+v+b \mathbf{i} \tau_{j}\right)\right] /$ $\left[\frac{1}{\pi} \sin \pi\left(\frac{b \kappa_{4}}{N}+v+b \mathrm{i} a_{32}^{S}-b^{2}\right) \prod_{t=1}^{N} \frac{1}{\pi} \sin \pi\left(v-b \mathrm{i} a_{1}^{t}\right)\right]$, which is equal to minus its residue at the last pole $v=b^{2}-\frac{b \kappa_{4}}{N}-b \mathrm{i} a_{32}^{s}$. That residue turns out to cancel most factors in the second to last line. Together, these last two lines of (4.24) are equal to

$$
\frac{\Gamma\left(b \kappa_{2}\right) \prod_{u \neq s}^{N} \Gamma\left(b \mathrm{i} a_{32}^{u}-b \mathrm{i} a_{32}^{s}\right)}{\prod_{t=1}^{N} \Gamma\left(\frac{b \kappa_{2}}{N}+b \mathrm{i} a_{3}^{t}-b \mathrm{i} a_{32}^{s}\right)}=\mathbf{F}_{s}\left[\begin{array}{cc}
\left(\kappa_{2}+b\right) h_{1} & -b h_{1} \\
\alpha_{3} & 2 Q-\alpha_{32}
\end{array}\right] .
$$

In particular, this does not depend on $\mathrm{i} \tau_{j}$ and can be pulled out of the integral. The first two lines of (4.24) then reproduce precisely the braiding matrix (4.4) of two semi-degenerate vertex operators. This concludes our check of the pentagon relation $(4.17)=(4.20)$.

This pentagon relation expresses the braiding kernel $\mathbf{B}_{\alpha_{12} \alpha_{32}}$ as a sum of $N$ braiding kernels with $\kappa_{2} \rightarrow \kappa_{2}+b, \alpha_{1} \rightarrow \alpha_{1}-b h_{p}$ and $\alpha_{32} \rightarrow \alpha_{32}^{\prime}=\alpha_{32}+b h_{s}$. Thus, if the braiding kernel was known for some value $\kappa_{2}=\lambda$, it could be deduced for $\kappa_{2}=\lambda-K b$ for integer $K \geq 0$. The pentagon identity $(1 \rightarrow 2 \rightarrow 3 \rightarrow 5)=(1 \rightarrow 4 \rightarrow 5)$ is checked through very similar computations. It allows the opposite shifts: from the $\kappa_{2}=\lambda$ braiding kernel one gets the $\kappa_{2}=\lambda+K b$ braiding kernel. By symmetry, identical shift relations exist with $b \rightarrow \frac{1}{b}$, thus fixing braiding kernels for $\kappa_{2}=\lambda+K b+L / b$ for all integers $K, L$. For generic real $b^{2}$, continuity would then determine the braiding kernel uniquely. Of course, this logic requires knowing for some $\kappa_{2}$ that the braiding kernel is correct for all values of other momenta.

From the trivial braiding kernel at $\kappa_{2}=0$ the shift relations allow us to reach all degenerate momenta $\kappa_{2}=-K b-L b^{-1}$ for $K, L \geq 0$, thus proving in particular that the braiding matrix (3.42) $(L=0)$ is correct. On the other hand, operators $\widehat{V}_{-K b-L b^{-1}}$ vanish if $K=-1$ or $L=-1$, which prevents us from using the pentagon relation to deduce prove our answer for $K<-1$ or $L<-1$.

To establish the proposed braiding kernel a strategy could be to derive shift relations on each momentum separately as was done in the Liouville case. We hope to return to this in the future. In the mean-time, the shift relations that we have already proven are at least very strong evidence that the proposed braiding kernel is correct. 


\section{Domain wall and its symmetries}

We want to describe the S-duality domain wall of $4 \mathrm{~d} \mathcal{N}=2 \mathrm{SU}(N)$ SQCD as a $3 \mathrm{~d} \mathcal{N}=2$ gauge theory coupled to the bulk on both sides. As explained in section 2, its $S_{b}^{3}$ (ellipsoid) partition function is equal, up to prefactors (2.8), to the $W_{N}$ braiding kernel (4.4) that we just computed. To warm up we explain in section 5.1 how continuous flavour symmetries of the $4 \mathrm{~d} / 3 \mathrm{~d}$ coupled systems given in the introduction reproduce those expected of the S-duality wall. Then we move on in section 5.2 to more involved expressions: comparing the braiding kernel to localization results. In fact, these are the formulas from which we read off the announced $3 \mathrm{~d} U(N-1)$ and $\operatorname{USp}(2 N-2)$ gauge theories. Section 5.3 matches $\mathbb{Z}_{2}$ symmetries of the $W_{N}$ braiding, of the abstract S-duality wall, and of its concrete $3 \mathrm{~d}$ gauge theory description.

Most interestingly, charge conjugation of $4 \mathrm{~d}$ theories is not a manifest symmetry of the $3 \mathrm{~d}$ theory. To show that our explicit expressions are invariant we lift the $3 \mathrm{~d}$ partition function of the $\operatorname{USp}(2 N-2)$ theory to a $4 \mathrm{~d}$ index then use Seiberg duality for USp groups. Alternatively, a physical understanding was obtained in [42] (after version 1 of this paper) where a variant of Aharony duality was found, under which our $\mathrm{U}(N-1)$ theory is self-dual. Finally, we discuss peculiarities for $N=2$ due to $\operatorname{USp}(2)=\mathrm{SU}(2)$.

\subsection{Continuous flavour symmetries}

Before turning to partition functions and how we obtained the following descriptions we explain how continuous flavour symmetries of the $4 \mathrm{~d} / 3 \mathrm{~d}$ coupled systems reproduce those expected of the S-duality wall. The two quiver descriptions are (with notations below)

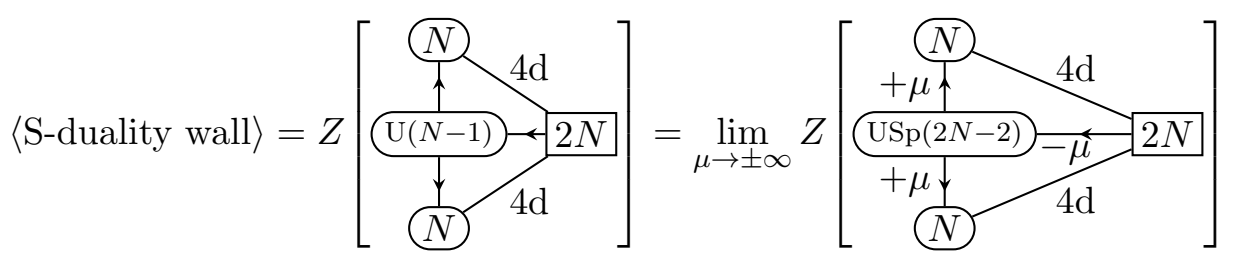

with superpotential

$$
W=\sum_{f=1}^{2 N} \sum_{s=1}^{N}\left(\left.\Phi_{f s}\right|_{3 \mathrm{~d}} \widetilde{q}_{s} q_{f}+\left.\Phi_{f s}^{\prime}\right|_{3 \mathrm{~d}} \widetilde{q}_{s}^{\prime} q_{f}\right)+ \begin{cases}V_{+}+V_{-} & \text {for the } \mathrm{U}(N-1) \text { theory } \\ Y & \text { for the } \mathrm{USp}(2 N-2) \text { theory }\end{cases}
$$

The quivers denote $4 \mathrm{~d} / 3 \mathrm{~d}$ coupled systems involving a $3 \mathrm{~d} \mathcal{N}=2$ gauge theory with $\mathrm{U}(N-1)$ or USp $(2 N-2)$ gauge group, $2 N$ fundamental chiral multiplets $q_{f}, N+N$ antifundamentals $\widetilde{q}_{s}, \widetilde{q}_{s}$, and a monopole superpotential made of all minimal monopoles $V_{ \pm}$or $Y$ for the given gauge group. The $\mathrm{SU}(N)$ flavour symmetries of $\widetilde{q}$ and $\widetilde{q}^{\prime}$ in this theory are each gauged using the $3 \mathrm{~d}$ restriction of the $4 \mathrm{~d} \mathcal{N}=2 \mathrm{SU}(N)$ vector multiplet on one side of the wall. The $3 \mathrm{~d}$ superpotential (5.2) is built using the $3 \mathrm{~d} \mathcal{N}=2$ chiral multiplets and using restrictions $\left.\Phi\right|_{3 \mathrm{~d}}$ and $\left.\Phi^{\prime}\right|_{3 \mathrm{~d}}$ of the $4 \mathrm{~d} \mathcal{N}=2$ hypermultiplets of both SQCD theories. It is compatible with the $\mathrm{SU}(N)^{2}$ gauging and breaks many other symmetries as explained next, identifying for example the $\mathrm{SU}(2 N)$ flavour symmetries of $q$ and of bulk hypermultiplets. 
The $\mathrm{U}(N-1)$ theory with $2 N$ flavours has an $\mathrm{SU}(2 N)_{1} \times \mathrm{SU}(2 N)_{2} \times \mathrm{U}(1)_{B} \times \mathrm{U}(1)_{T}$ flavour symmetry and $\mathrm{U}(1)_{R}$ symmetry. Gauging an $\mathrm{SU}(N)^{2}$ subgroup of one $\mathrm{SU}(2 N)_{1}$ using the two $4 \mathrm{~d}$ gauge symmetries reduces that factor of the flavour symmetry to $\mathrm{U}(1)_{1}$. Besides being consistent with this identification, the superpotential breaks many $3 \mathrm{~d}$ and $4 \mathrm{~d}$ symmetries to their diagonal subgroup, thus identifying pairs of symmetries.

- The 3 d symmetry $\mathrm{SU}(2 N)_{2}$ is identified to the $\mathrm{SU}(2 N)$ flavour symmetries of the two $4 \mathrm{~d}$ theories by the first two terms in $W$. This reproduces the fact that S-duality identifies the $\mathrm{SU}(2 N)$ flavour symmetries of dual theories.

- The first two terms in $W$ further identify the $\mathrm{U}(1)_{1}$ symmetry to the difference of baryonic $\mathrm{U}(1)$ flavour symmetries of the two $4 \mathrm{~d}$ theories, and the $3 \mathrm{~d}$ baryonic symmetry $\mathrm{U}(1)_{B}$ to their sum.

- The dressed monopole operators $V_{+}$and $V_{-}$have the same non-zero charge under the $\mathrm{U}(1)_{B}$ flavour symmetry of the $3 \mathrm{~d}$ theory and opposite charges under the topological one, so both $\mathrm{U}(1)_{B}$ and $\mathrm{U}(1)_{T}$ are broken by the superpotential terms $V_{+}+V_{-}$. In particular, only the difference of $4 \mathrm{~d}$ baryonic symmetries survives (further combined with $\mathrm{U}(1)_{1}$ as seen above). This reproduces the fact that the baryonic symmetry is flipped by S-duality.

Furthermore, $W$ has $R$-charge 2 under the $\mathrm{U}(1)_{R}$ symmetry of $3 \mathrm{~d} \mathcal{N}=2$. This is a subgroup of the $\mathrm{SU}(2)_{R}$ symmetry of $4 \mathrm{~d} \mathcal{N}=2$, so $\mathrm{U}(1)_{R}$-charges of the $4 \mathrm{~d}$ fields $\Phi$ and $\Phi^{\prime}$ are integers. By continuity these charges on $S_{b}^{4}$ must be equal to those in flat space, which are 1 since hypermultiplet scalars are in a doublet of $\mathrm{SU}(2)_{R}$. As a result, the $R$-charges of $q$ and $\widetilde{q}$ must sum to 1 , as do those of $q$ and $\widetilde{q}$. Up to a diagonal gauge redundancy, the superpotential thus sets $R$-charges of all $3 \mathrm{~d}$ chiral multiplets to their canonical UV value $\frac{1}{2}$. Since none of the remaining unbroken U(1) symmetries leave all chiral multiplets invariant, we learn that the UV and IR $R$-symmetries coincide. A consistency check is that under this UV $R$-symmetry of the $3 \mathrm{~d}$ theory the monopole operators $V_{ \pm}$also have the correct $R$-charge. ${ }^{17}$ Symmetries and charges are summarized in table 2 .

The USp $(2 N-2)$ theory is similar. The fundamental and antifundamental representations of USp are isomorphic, but gauging $\mathrm{SU}(N)^{2}$ breaks the large flavour symmetry down to $\mathrm{U}(1)_{1} \times \mathrm{SU}(2 N)_{2} \times \mathrm{U}(1)_{B} \times \mathrm{U}(1)_{A}$, where $\mathrm{U}(1)_{B}$ and $\mathrm{U}(1)_{A}$ act on $(q, \widetilde{q}, \widetilde{q})$ with charges $( \pm 1,+1,+1)$. The axial flavour symmetry $\mathrm{U}(1)_{A}$ is new here because it was gauged in the previous model. As above, the superpotential identifies $\mathrm{SU}(2 N)_{2}$ with both $\mathrm{SU}(2 N)$ $4 \mathrm{~d}$ flavour symmetries, and identifies $\mathrm{U}(1)_{B}$ and $\mathrm{U}(1)_{1}$ with the sum and difference of $4 \mathrm{~d}$ baryonic symmetries, respectively. The monopole term $Y$ is charged under $\mathrm{U}(1)_{B}$ hence breaks this symmetry. Fixing the $R$-charge of $W$ to be 2 makes $q, \widetilde{q}, \widetilde{q}^{\prime}$ have canonical $R$-charges up to mixing the $R$-symmetry with $\mathrm{U}(1)_{A}$ (which acts trivially on $4 \mathrm{~d}$ fields). As

\footnotetext{
${ }^{17}$ Alternatively, one can start by analyzing the $\mathrm{U}\left(N_{c}\right)$ theory with $N_{f}$ flavours and monopole superpotential, as done in [42]. The superpotential sets the $R$-charge of monopoles to 2 , which fixes most possible mixing of $R$-symmetry with other symmetries, thus setting $R$-charges of $q, \widetilde{q}, \widetilde{q}^{\prime}$ to their UV value. In this approach, the consistency check is that the cubic superpotential coupling with $\left.\Phi\right|_{3 \mathrm{~d}}$ and $\left.\Phi^{\prime}\right|_{3 \mathrm{~d}}$ has the correct $R$-charge 2 .
} 


\begin{tabular}{|cccccccc|}
\hline \multicolumn{3}{c}{ Gauge symmetries } & & \multicolumn{3}{c|}{ Global symmetries } \\
\cline { 2 - 3 } \cline { 6 - 7 } & $\mathrm{U}(N-1) \times \mathrm{SU}(N)_{1} \times \mathrm{SU}(N)_{2}$ & & $\mathrm{U}(1)_{1} \times \mathrm{SU}(2 N)_{2} \times \mathrm{U}(1)_{R}$ \\
\hline$q$ & $\overline{N-1}$ & 1 & 1 & & 0 & $2 N$ & $\frac{1}{2}$ \\
$\widetilde{q}$ & $N-1$ & $\bar{N}$ & 1 & & +1 & 1 & $\frac{1}{2}$ \\
$\widetilde{q}$ & $N-1$ & 1 & $\bar{N}$ & & -1 & 1 & $\frac{1}{2}$ \\
$V_{ \pm}$ & 1 & 1 & 1 & & 0 & 1 & 2 \\
$\Phi$ & 1 & $N$ & 1 & & -1 & $\overline{2 N}$ & 1 \\
$\Phi^{\prime}$ & 1 & 1 & $N$ & & +1 & $\overline{2 N}$ & 1 \\
\hline
\end{tabular}

\begin{tabular}{|c|c|c|c|c|c|c|c|}
\hline \multicolumn{4}{|c|}{ Gauge symmetries } & \multicolumn{4}{|c|}{ Global symmetries } \\
\hline \multicolumn{4}{|c|}{$\mathrm{USp}(2 N-2) \times \mathrm{SU}(N)_{1} \times \mathrm{SU}(N)_{2}$} & \multicolumn{4}{|c|}{$\mathrm{U}(1)_{1} \times \mathrm{SU}(2 N)_{2} \times \mathrm{U}(1)_{A} \times \mathrm{U}(1)_{R}$} \\
\hline$q$ & $2 N-2$ & 1 & 1 & 0 & $2 N$ & -1 & $\frac{1}{2}-\nu$ \\
\hline$\widetilde{q}$ & $2 N-2$ & $\bar{N}$ & 1 & +1 & 1 & +1 & $\frac{1}{2}+\nu$ \\
\hline$\widetilde{q}^{\prime}$ & $2 N-2$ & 1 & $\bar{N}$ & -1 & 1 & +1 & $\frac{1}{2}+\nu$ \\
\hline$Y$ & 1 & 1 & 1 & 0 & 1 & 0 & 2 \\
\hline$\Phi$ & 1 & $N$ & 1 & -1 & $\overline{2 N}$ & 0 & 1 \\
\hline$\Phi^{\prime}$ & 1 & 1 & $N$ & +1 & $\overline{2 N}$ & 0 & 1 \\
\hline
\end{tabular}

Table 2. Charges of $3 \mathrm{~d}$ and $4 \mathrm{~d}$ fields under unbroken symmetries for the two descriptions of the S-duality domain wall in (5.1). First we list gauge groups of the $3 \mathrm{~d}$ theory and $4 \mathrm{~d}$ theories, then flavour and $R$-symmetry groups. Charges coincide except for the additional symmetry $\mathrm{U}(1)_{A}$ in the second case, and the possibility to mix it into the $R$-symmetry for some parameter $\nu$ with $|\nu| \leq 1 / 4$.

summarized in table 2, symmetries are identical to the previous model, except for the extra symmetry $\mathrm{U}(1)_{A}$. To eliminate $\mathrm{U}(1)_{A}$ we turn on a mass parameter $\mu$ for it, namely add $\pm \mu$ to the masses of chiral multiplets as indicated by the markings $+\mu$ and $-\mu$ in (5.1). We then take $\mu \rightarrow+\infty$. We will find that the $S_{b}^{3}$ partition function reduces to contributions from the neighborhood of a point $\tau_{\mu}=(\mu, \ldots, \mu,-\mu, \ldots,-\mu)$ of the Coulomb branch where only half of the chiral multiplets acquire a large mass $2 \mu$. The gauge group is reduced to $\mathrm{U}(N-1)$, and every fundamental chiral multiplet of $\operatorname{USp}(2 N-2)$ splits into a fundamental and an antifundamental one, one of which acquires a mass. The $\mu \rightarrow \pm \infty$ limit is then the $\mathrm{U}(N-1) 3 \mathrm{~d}$ theory in (5.1). A separate concern caused by $\mathrm{U}(1)_{A}$ is that the $R$-symmetry of $3 \mathrm{~d} \mathcal{N}=2$ can mix with $\mathrm{U}(1)_{A}$ along the RG flow, and one should perform $F$-extremization [38] for each value of $\mu$ to determine the IR $R$-charges. Very limited numerical tests suggests that $R$-charges remain bounded, so that their $\mu$ dependence does not affect the limit. 


\subsection{Partition function}

\subsection{1 $\mathrm{U}(N-1)$ description}

The coefficients in (2.8) are products of $1 / \Upsilon_{b}(x)=\Gamma_{b}(x) \Gamma_{b}\left(b+b^{-1}-x\right)$ which combine with prefactors of the braiding kernel (4.4). Toda CFT momenta are converted to gauge theory parameters $a$ and $a^{\prime}$ and hypermultiplet masses $m_{f}(f=1, \ldots, 2 N)$ using the dictionary (2.10), $\alpha_{12}=Q+\sum_{j=1}^{N} \mathrm{i} a_{j} h_{j}$ and $\alpha_{32}=Q-\sum_{j=1}^{N} \mathrm{i} a_{j}^{\prime} h_{j}$. Denote $m=\sum_{f=1}^{2 N} m_{f} /(2 N)$, write $m_{f}=m+\hat{m}_{f}$, shift all $\tau_{j}$ and use $1 / S_{b}(x)=S_{b}\left(b+b^{-1}-x\right)$ to find what partition function to expect given our Toda CFT results:

$$
\begin{aligned}
& \left\langle\text { S-duality wall on } S_{b}^{3} \subset S_{b}^{4}\right\rangle \\
& =Z_{1 \text {-loop }}^{\text {half-ellipsoid }}(m, a) Z_{1 \text {-loop }}^{\text {halfellipsoid }}\left(m, a^{\prime}\right) Z_{S_{b}^{3}}\left(m, a, a^{\prime}\right) \\
& =\frac{\prod_{f=1}^{2 N} \prod_{t=1}^{N} \Gamma_{b}\left(\frac{b+b^{-1}}{2}+\mathrm{i} \hat{m}_{f}+\mathrm{i} m-\mathrm{i} a_{t}\right)}{\prod_{s \neq t}^{N} \Gamma_{b}\left(\mathrm{i} a_{t}-\mathrm{i} a_{s}\right)} \frac{\prod_{f=1}^{2 N} \prod_{t=1}^{N} \Gamma_{b}\left(\frac{b+b^{-1}}{2}+\mathrm{i} \hat{m}_{f}-\mathrm{i} m-\mathrm{i} a_{t}^{\prime}\right)}{\prod_{s \neq t}^{N} \Gamma_{b}\left(\mathrm{i} a_{t}^{\prime}-\mathrm{i} a_{s}^{\prime}\right)} \\
& \quad \times \int \frac{\mathrm{d}^{N-1} \tau}{\prod_{i \neq j}^{N-1} S_{b}\left(\mathrm{i} \tau_{i}-\mathrm{i} \tau_{j}\right)} \prod_{j=1}^{N-1}\left[\prod_{f=1}^{2 N} S_{b}\left(\frac{b+b^{-1}}{4}-\mathrm{i} \hat{m}_{f}+\mathrm{i} \tau_{j}\right)\right. \\
& \left.\quad \times \prod_{s=1}^{N} S_{b}\left(\frac{b+b^{-1}}{4}-\mathrm{i} m+\mathrm{i} a_{s}-\mathrm{i} \tau_{j}\right) \prod_{s=1}^{N} S_{b}\left(\frac{b+b^{-1}}{4}+\mathrm{i} m+\mathrm{i} a_{s}^{\prime}-\mathrm{i} \tau_{j}\right)\right] .
\end{aligned}
$$

Reassuringly, despite the asymmetry between masses $m_{1}, \ldots, m_{N}$ and $m_{N+1}, \ldots, m_{2 N}$ in Toda CFT expressions, the final expression is invariant under permutations of these masses.

The first line in (5.3) gives perfect candidates for the one-loop determinant of a hypermultiplet on a half-ellipsoid and that of a vector muliplet,

$$
Z_{1-\text { loop, hyper }}^{\text {half-ellipsoid }}(m)=\Gamma_{b}\left(\frac{b+b^{-1}}{2}+\mathrm{i} m\right) \quad Z_{1-\text { loop, vector }}^{\text {half-ellipsoid }}(a)=\prod_{e \in\{\text { roots }\}} \frac{1}{\Gamma_{b}(\mathrm{i}\langle e \mid a\rangle)}
$$

where $\langle e \mid a\rangle$ is the usual scalar product of roots with elements of the Cartan algebra. These candidates appear to be consistent with results on the full ellipsoid: indeed,

$$
\begin{aligned}
Z_{1-\text { loop, hyper }}^{S_{4}^{4}}(m) & =Z_{1-\text { loop, hyper }}^{\text {half } S_{b}^{4}}(m) Z_{1 \text {-loop, hyper }}^{\text {half } S_{b}^{4}}(-m) \\
Z_{1 \text {-loop, vector }}^{S_{b}^{4}}(a) & =Z_{1 \text {-loop, vector }}^{\text {half } S_{b}^{4}}(a) Z_{1 \text {-loop, vector }}^{\text {half } S_{b}^{4}}(-a) / Z_{1 \text {-loop, vector }}^{S_{b}^{3}}(a) .
\end{aligned}
$$

More precisely, the vector multiplet one-loop determinant is given here for the case of Neumann boundary conditions (namely gauge transformations are not frozen at the boundary). The need to divide by the one-loop determinant of a vector multiplet on $S_{b}^{3}$ is not surprising since we would otherwise be overcounting degrees of freedom.

We are left with the task of finding a $3 \mathrm{~d} \mathcal{N}=2$ gauge theory whose $S_{b}^{3}$ partition function is the integral in (5.3). Such partition functions are known through supersymmetric localization [38-40]: the path integral is localized to field configurations where a real vector multiplet scalar takes an arbitrary constant value, which can be reduced to the 
Cartan algebra of the gauge group $G$ by a gauge transformation. The partition function takes the form

$$
Z_{S_{b}^{3}}(\underline{f})=\int_{-\infty}^{\infty} \prod_{j=1}^{\operatorname{rank} G}\left[\mathrm{~d} \tau_{j} e^{\pi \mathrm{i} k \tau_{j}^{2}} e^{-2 \pi \lambda \tau_{j}}\right] \frac{\prod_{I} \prod_{w_{I} \in \mathcal{R}_{I}} S_{b}\left(\frac{1}{2}\left(b+b^{-1}\right) r_{I}+\mathrm{i}\left\langle w_{I} \mid \tau\right\rangle+\mathrm{i} m_{I}\right)}{\prod_{e \in\{\text { roots }\}} S_{b}(\mathrm{i}\langle e \mid \tau\rangle)},
$$

where the exponentials are classical values of the action, with $k$ the Chern-Simons level (one per simple factor of $G$ ) and $\lambda$ the Fayet-Iliopoulos parameter (one per abelian factor of $G$ ), the product over roots $e$ of $G$ is the one-loop contribution of the vector multiplet, and finally each chiral multiplet transforming in a representation $\mathcal{R}_{I}$ of $G$ contributes a product over weights $w_{I}$ including multiplicity (we write $w_{I} \in \mathcal{R}_{I}$ for lack of a better notation), which involves the $R$-charge $r_{I}$ and mass $m_{I}$ of the chiral. The integration contour of each $\tau_{j}$ agrees with $\mathbb{R}$ away from a compact set and is chosen so that for each $S_{b}$ all the poles are above the contour or all below. Note that the vector multiplet contribution has no pole since $\left(S_{b}(y) S_{b}(-y)\right)^{-1}=-4 \sin (\pi b y) \sin \left(\pi b^{-1} y\right)$.

We now find what $3 \mathrm{~d} \mathcal{N}=2$ theory reproduces (5.3) as follows. From the product of $S_{b}\left(\mathrm{i} \tau_{i}-\mathrm{i} \tau_{j}\right)$ which does not depend on masses we deduce that the gauge group is $\mathrm{U}(N-1)$. All remaining $S_{b}$ functions are one-loop determinants of chiral multiplets with canonical $R$-charge $\frac{1}{2}$ (since the arguments take the form $\left.\frac{1}{4}\left(b+b^{-1}\right)+\cdots\right)$. The multiplets are $2 N$ fundamentals $q_{f}$ of $\mathrm{U}(N-1)$ with masses $-\hat{m}_{f}$ for $f=1, \ldots, 2 N$, and $2 N$ antifundamentals $\widetilde{q}_{s}$ and $\widetilde{q}_{s}^{\prime}$ with masses $a_{s}-m$ and $a_{s}^{\prime}+m$ for $s=1, \ldots, N$. These masses and $R$-charges are consistent with coupling the $3 \mathrm{~d}$ and $4 \mathrm{~d}$ matter multiplets along the defect using the cubic superpotential

$$
W_{\text {cubic }}=\sum_{f=1}^{2 N} \sum_{s=1}^{N}\left(\left.\Phi_{f s}\right|_{3 \mathrm{~d}} \widetilde{q}_{s} q_{f}+\left.\Phi_{f s}^{\prime}\right|_{3 \mathrm{~d}} \widetilde{q}_{s} q_{f}\right) .
$$

Here $\left.\Phi\right|_{3 \mathrm{~d}}$ and $\left.\Phi^{\prime}\right|_{3 \mathrm{~d}}$ are restrictions of $4 \mathrm{~d}$ hypermultiplets at the interface; more precisely they are the $3 \mathrm{~d} \mathcal{N}=2$ chiral multiplet whose bottom component is the $3 \mathrm{~d}$ restriction of one complex scalar in the hypermultiplets. The $R$-charge of these fields originating from $4 \mathrm{~d}$ must be an integer since the $3 \mathrm{~d} \mathrm{U}(1)_{R}$ symmetry is embedded in the non-Abelian $\mathrm{SU}(2)_{R}$. It must be precisely 1 because in flat space the scalars in $4 \mathrm{~d}$ hypermultiplets transform in a doublet of $\mathrm{SU}(2)_{R}$. Therefore every term in the superpotential has $R$-charge 2 . It is immediate to check that other charges sum to zero for each term: $\left(\hat{m}_{f}+m-a_{s}\right)+\left(a_{s}-\right.$ $m)+\left(-\hat{m}_{f}\right)=0$ and $\left(\hat{m}_{f}-m-a_{s}^{\prime}\right)+\left(a_{s}^{\prime}+m\right)+\left(-\hat{m}_{f}\right)=0$.

As discussed in section 5.1 the superpotential identifies $\mathrm{SU}(2 N)$ symmetries of the $3 \mathrm{~d}$ and $4 \mathrm{~d}$ theories, and $3 \mathrm{~d} \mathrm{SU}(N)$ symmetries to $4 \mathrm{~d}$ gauge symmetries. It also breaks some $\mathrm{U}(1)$ symmetries, but leaves four:

- $\mathrm{U}(1)_{R}$, whose effect on the arguments of $S_{b}$ functions is to include a term $\frac{1}{4}\left(b+b^{-1}\right)$,

- $\mathrm{U}(1)_{1}$, whose mass parameter $m$ we see in masses $a_{s}-m$ and $a_{s}^{\prime}+m$ of antifundamental chiral multiplets,

- $\mathrm{U}(1)_{B}$, whose mass parameter would appear with the same sign in all $S_{b}$ functions,

- $\mathrm{U}(1)_{T}$, whose mass parameter (the FI parameter) would appear as an exponential contribution $e^{-2 \pi \lambda \sum_{j} \tau_{j}}$. 
We thus need to add to the superpotential some terms that break $\mathrm{U}(1)_{B}$ and $\mathrm{U}(1)_{T}$. It turns out that the monopole operators $V_{ \pm}$do the trick, giving the superpotential (1.3).

As explained at the end of section 2.2, if instanton partition functions (conformal blocks) are normalized to have a leading term $x^{\cdots}$ rather than $(-x)^{\cdots}$, then the S-duality kernel is changed by phases (2.13) $\exp \left(\epsilon \mathrm{i} \pi\left[\frac{1}{2} \sum_{j} a_{j}^{2}+\frac{1}{2} \sum_{j} a_{j}^{\prime 2}\right]\right)$ depending on the halfplane $(\epsilon= \pm 1$ is the sign of $\operatorname{Im} x)$. These phases are reproduced by a Chern-Simons term of level $\frac{1}{2}$ for $3 \mathrm{~d}$ restrictions of the $4 \mathrm{~d}$ gauge $\mathrm{SU}(N)$ fields.

\subsubsection{USp $(2 N-2)$ description}

We now consider the $\operatorname{USp}(2 N-2)$ theory in (5.1) and explain its partition function has the $\mathrm{U}(N-1)$ one as a limit, up to a divergent factor $e^{N(N-1) \pi\left(b+b^{-1}\right) \mu}$ omitted here. We wish to take $\mu \rightarrow \infty$ in the $S_{b}^{3}$ partition function

$$
\begin{aligned}
Z_{S_{b}^{3}}= & \int \mathrm{d}^{N-1} \tau \prod_{ \pm}\left\{\frac{\prod_{j=1}^{N-1} \prod_{f=1}^{2 N} S_{b}\left(\frac{b+b^{-1}}{4}-\mathrm{i} \hat{m}_{f}-\mathrm{i} \mu \pm \mathrm{i} \tau_{j}\right)}{\prod_{i \leq j}^{N-1} S_{b}\left( \pm\left(\mathrm{i} \tau_{i}+\mathrm{i} \tau_{j}\right)\right) \prod_{i<j}^{N-1} S_{b}\left( \pm\left(\mathrm{i} \tau_{i}-\mathrm{i} \tau_{j}\right)\right)}\right. \\
& \left.\times \prod_{j=1}^{N-1} \prod_{s=1}^{N}\left[S_{b}\left(\frac{b+b^{-1}}{4}-\mathrm{i} m+\mathrm{i} a_{s}+\mathrm{i} \mu \pm \mathrm{i} \tau_{j}\right) S_{b}\left(\frac{b+b^{-1}}{4}+\mathrm{i} m+\mathrm{i} a_{s}^{\prime}+\mathrm{i} \mu \pm \mathrm{i} \tau_{j}\right)\right]\right\} .
\end{aligned}
$$

From the asymptotics of $\Gamma_{b}[57$, Proposition 8.11] we work out that for $|\chi| \rightarrow \pm \infty$ away from the imaginary axis,

$S_{b}(A+\mathrm{i} \chi) S_{b}(B-\mathrm{i} \chi) \sim e^{-\pi\left(b+b^{-1}-A-B\right) \chi \operatorname{sign}(\operatorname{Re} \chi)} e^{\operatorname{sign}(\operatorname{Re} \chi) \frac{\mathrm{i} \pi}{2}\left(\left(\frac{1}{2}\left(b+b^{-1}\right)-B\right)^{2}-\left(\frac{1}{2}\left(b+b^{-1}\right)-A\right)^{2}\right)}$.

We will always take $\chi$ real so $\chi \operatorname{sign}(\operatorname{Re} \chi)=|\chi|$.

Let us apply (5.10) to pairs of $S_{b}$ functions in (5.9) which have opposite dependence on $\mu$ and $\tau_{j}$, taking $A$ and $B$ to be everything apart from $\mu$ and $\tau_{j}$. We ignore for now factors that are uniformly bounded functions of $\mu$ and $\tau_{j}$ (and have uniformly bounded inverse), and will denote them by (finite). This allows us to keep only the first exponential in (5.10). In fact, $S_{b}(A+\mathrm{i} \chi) S_{b}(B-\mathrm{i} \chi)=e^{-\pi\left(b+b^{-1}-A-B\right)|\chi|}($ finite). The integrand becomes

$$
\text { (finite) } \exp \left(\pi\left(b+b^{-1}\right)\left(-N \sum_{j=1}^{N-1}\left(\left|\mu-\tau_{j}\right|+\left|\mu+\tau_{j}\right|\right)+\sum_{i \leq j}^{N-1}\left|\tau_{i}+\tau_{j}\right|+\sum_{i<j}^{N-1}\left|\tau_{i}-\tau_{j}\right|\right)\right) \text {. }
$$

Now $\left|\mu-\tau_{j}\right|+\left|\mu+\tau_{j}\right|=\max \left(2|\mu|, 2\left|\tau_{j}\right|\right)$ and $\left|\tau_{i}-\tau_{j}\right|+\left|\tau_{i}+\tau_{j}\right|=\max \left(2\left|\tau_{i}\right|, 2\left|\tau_{j}\right|\right)$. Sorting the parameters as $\left|\tau_{1}\right|<\ldots<\left|\tau_{I}\right|<|\mu|<\left|\tau_{I+1}\right|<\cdots<\left|\tau_{N-1}\right|$, the exponential is

$$
\exp \left(\pi\left(b+b^{-1}\right)\left(-N(N-1) \mu-2 \sum_{j=1}^{I} j\left(|\mu|-\left|\tau_{j}\right|\right)-2 \sum_{j=I+1}^{N-1}(N-j)\left(\left|\tau_{j}\right|-|\mu|\right)\right)\right) .
$$

The second and third terms are negative. Hence the dominant contribution to the integral (5.9) as $\mu \rightarrow \infty$ is when $\left|\tau_{j}\right|-|\mu|$ are finite, and away from these regions the integrand decays exponentially. 
We can now go back and keep finite factors when expanding the integrand in the region $\left|\tau_{j}\right| \sim|\mu|$. Given symmetries under $\tau_{j} \rightarrow-\tau_{j}$, we focus on the case $\tau_{j}=\mu+\hat{\tau}_{j}$ with $\hat{\tau}_{j}$ finite. Half of the $S_{b}$ factors remain finite and form the partition function of the $\mathrm{U}(N-1)$ theory, while the other half can be paired and turned into exponentials through (5.10). One gets

$$
\begin{aligned}
& \lim _{\mu \rightarrow \pm \infty}\left[E\left(\mu, b, m, \hat{m}, a, a^{\prime}\right) Z_{S_{b}^{3}}(\mathrm{USp}(2 N-2) \text { theory })\right] \\
& =\int \mathrm{d}^{N-1} \hat{\tau}\left\{\frac{\prod_{j=1}^{N-1} \prod_{f=1}^{2 N} S_{b}\left(\frac{b+b^{-1}}{4}-\mathrm{i} \hat{m}_{f}+\mathrm{i} \hat{\tau}_{j}\right)}{\prod_{ \pm} \prod_{i<j}^{N-1} S_{b}\left( \pm\left(\mathrm{i} \hat{\tau}_{i}-\mathrm{i} \hat{\tau}_{j}\right)\right)}\right. \\
& \left.\quad \times \prod_{j=1}^{N-1} \prod_{s=1}^{N}\left[S_{b}\left(\frac{b+b^{-1}}{4}-\mathrm{i} m+\mathrm{i} a_{s}-\mathrm{i} \hat{\tau}_{j}\right) S_{b}\left(\frac{b+b^{-1}}{4}+\mathrm{i} m+\mathrm{i} a_{s}^{\prime}-\mathrm{i} \hat{\tau}_{j}\right)\right]\right\} \\
& =Z_{S_{b}^{3}}(\mathrm{U}(N-1) \text { theory }) .
\end{aligned}
$$

where $E=\left[\frac{1}{2} e^{N \pi\left(b+b^{-1}\right)|\mu|} e^{\operatorname{sign}(\mu) \frac{i \pi}{2}\left(\sum_{f=1}^{2 N} \hat{m}_{f}^{2}-\sum_{s=1}^{N} a_{s}^{2}-\sum_{s=1}^{N} a_{s}^{\prime 2}-2 N m^{2}\right)}\right]^{N-1}$. As announced, the partition function of the $\mathrm{U}(N-1)$ theory is a limit of the partition function of the $\mathrm{USp}(2 N-2)$ theory. See [42] for the analogous statement for the theories themselves, although one may want to keep track of the factor $E$, which consists of Chern-Simons terms for all flavour symmetry groups of the $3 \mathrm{~d}$ theories.

\subsection{Discrete symmetries and dualities}

We now have all the necessary tools to study symmetries, from the point of view of the duality wall, of its $3 \mathrm{~d}$ gauge theory description, and of the Toda CFT. Symmetries of Toda CFT correlators (hence of their braiding kernel) may be the least familiar to the reader, so we will describe them and give their gauge theory interpretation.

Any vertex operator $\widehat{V}_{\alpha}$ is invariant under Weyl transformations, namely permutations of the components $\left\langle\alpha-Q, h_{s}\right\rangle$. Applying this symmetry to $\widehat{V}_{\alpha_{1}}$ permutes the first $N$ masses, and applying it to $\widehat{V}_{\alpha_{3}}$ permutes the last $N$. These are manifest symmetries of the S-duality wall and of its $3 \mathrm{~d}$ gauge theory description. In fact, permuting all of the $2 N$ masses is also a symmetry, but the Toda CFT description does not make all permutations manifest.

Conformal blocks are invariant under conjugating all momenta, which maps (up to a Weyl transformation) $\alpha \rightarrow 2 Q-\alpha$ and $\kappa h_{1} \rightarrow\left(N\left(b+b^{-1}\right)-\kappa\right) h_{1}$ hence flips the sign of all $m_{f}, a_{s}$ and $a_{s}^{\prime}$. This is the effect of charge conjugation in the $4 \mathrm{~d}$ theories on both sides of the wall, which is an expected symmetry of the S-duality wall. However, the effect is harder to describe in the $3 \mathrm{~d}$ gauge theory description, because this theory is chiral, hence not invariant a priori under charge conjugation. Correspondingly, the explicit form of the braiding kernel does not appear invariant under charge conjugation. Most of this section will be spent proving the invariance, which will turn out to be a limit of USp-type Seiberg duality of $4 \mathrm{~d} \mathcal{N}=1$ indices. Before proceeding let us describe two more symmetries.

Conformal blocks are also invariant under some permutations of the operators. In particular, the braiding kernel is thus invariant under a permutation obtained by rotating 
the diagrams by $180^{\circ}$ in the plane:

$$
\mathbf{B}_{\alpha_{12}, \alpha_{32}}\left[\begin{array}{cc}
\kappa_{4} h_{1} & \kappa_{2} h_{1} \\
\alpha_{3} & \alpha_{1}
\end{array}\right]=\mathbf{B}_{2 Q-\alpha_{12}, 2 Q-\alpha_{32}}\left[\begin{array}{cc}
\kappa_{2} h_{1} & \kappa_{4} h_{1} \\
\alpha_{1} & \alpha_{3}
\end{array}\right] \text { due to } \underset{\underset{\kappa_{4} h_{1} \uparrow}{\alpha_{3}} \underbrace{\alpha_{12} \downarrow \kappa_{2} h_{1}}_{\alpha_{1}}}{\stackrel{\leftarrow}{\rightleftarrows}}
$$

The map $\alpha_{12} \rightarrow 2 Q-\alpha_{12}$ is due to the arrow being reversed by the rotation. Composing with conjugation of all momenta yields the transformation $\alpha_{1} \leftrightarrow 2 Q-\alpha_{3}$ and $\kappa_{2} \leftrightarrow$ $N\left(b+b^{-1}\right)-\kappa_{4}$, which given the dictionary (2.10) simply exchanges the first $N$ and the last $N$ masses.

Lastly, the S-duality wall and its 3d gauge theory description are invariant under exchanges of the two hemispheres. This symmetry is manifest in explicit expressions, but on the Toda side it is not immediately clear why it should hold. The symmetry maps $a_{s} \leftrightarrow a_{s}^{\prime}$ and $m \rightarrow-m$. Momenta are mapped as $\kappa_{2} \leftrightarrow \kappa_{4}$ and $\alpha_{12} \leftrightarrow \alpha_{32}$, and the braiding kernel becomes the kernel for the inverse braiding, which expresses u-channel blocks in terms of s-channel ones. In addition to being inverses of each other, these braiding kernels are actually also equal up to some structure constants. To prove this, write the s-channel and $\mathrm{u}$-channel decompositions of the four-point function, then use braiding kernels to get an expression with a holomorphic s-channel block and an antiholomorphic u-channel block, and match coefficients of $\mathcal{F}_{\alpha_{12}}^{(\mathrm{s})}(x) \mathcal{F}_{\alpha_{32}}^{(\mathrm{u})}(\bar{x})$ :

$$
\begin{aligned}
& \langle\widehat{V} \widehat{V} \widehat{V} \widehat{V}\rangle=\int \mathrm{d} \alpha_{12} C^{(\mathrm{s})} \mathcal{F}_{\alpha_{12}}^{(\mathrm{s})}(x) \mathcal{F}_{\alpha_{12}}^{(\mathrm{s})}(\bar{x})=\int \mathrm{d} \alpha_{12} \mathrm{~d} \alpha_{32} C^{(\mathrm{s})} \mathcal{F}_{\alpha_{12}}^{(\mathrm{s})}(x) \mathbf{B}_{\alpha_{12} \alpha_{32}} \mathcal{F}_{\alpha_{32}}^{(\mathrm{u})}(\bar{x}) \\
& \langle\widehat{V} \widehat{V} \widehat{V} \widehat{V}\rangle=\int \mathrm{d} \alpha_{32} C^{(\mathrm{u})} \mathcal{F}_{\alpha_{32}}^{(\mathrm{u})}(x) \mathcal{F}_{\alpha_{32}}^{(\mathrm{u})}(\bar{x})=\int \mathrm{d} \alpha_{12} \mathrm{~d} \alpha_{32} C^{(\mathrm{u})} \mathbf{B}_{\alpha_{32} \alpha_{12}}^{\kappa_{2} \leftrightarrow \kappa_{4}} \mathcal{F}_{\alpha_{12}}^{(\mathrm{s})}(x) \mathcal{F}_{\alpha_{32}}^{(\mathrm{u})}(\bar{x})
\end{aligned}
$$

These two decompositions into the basis of functions $\mathcal{F}_{\alpha_{12}}^{(\mathrm{s})}(x) \mathcal{F}_{\alpha_{32}}^{(\mathrm{u})}(\bar{x})$ must coincide hence $C^{(\mathrm{s})} \mathbf{B}_{\alpha_{12} \alpha_{32}}=C^{(\mathrm{u})} \mathbf{B}_{\alpha_{32} \alpha_{12}}^{\kappa_{2} \leftrightarrow \kappa_{4}}$. The structure constants $C^{(\mathrm{s})}$ and $C^{(\mathrm{u})}$ turn out to cancel with the prefactors relating the braiding kernel to the S-duality wall partition function so that we get an equality of wall partition functions.

Now that we have described all manifest symmetries of the explicit braiding kernel (4.4), we must tackle invariance under charge conjugation.

We will use identities of hyperbolic hypergeometric integrals [58]. The hyperbolic Gamma function $\Gamma_{h}^{(2)}$ of that paper reduces to our $S_{b}$ upon taking $\omega_{1} / \omega_{2}=b^{2}$. For definiteness, we take $\omega_{1}=\mathrm{i} b$ and $\omega_{2}=\mathrm{i} / b$ and note $S_{b}(x)=\Gamma_{h}^{(2)}(\mathrm{i} x ; \mathrm{i} b, \mathrm{i} / b)$. The $B C_{n}$ hyperbolic hypergeometric integral is defined by (to avoid factors of $\mathrm{i}$ we let $\mu_{r}=\mathrm{i} \nu_{r}$ )

$$
I_{B C_{n} ; h}^{(m)}\left(\nu_{0}, \ldots, \nu_{2 m+2 n+3}\right)=\frac{1}{2^{n} n !} \int_{\mathbb{R}^{n}} \mathrm{~d}^{n} x \prod_{ \pm} \frac{\prod_{i=1}^{n} \prod_{r=0}^{2 m+2 n+3} S_{b}\left(\nu_{r} \pm \mathrm{i} x_{i}\right)}{\prod_{i \leq j}^{n} S_{b}\left( \pm\left(\mathrm{i} x_{i}+\mathrm{i} x_{j}\right)\right) \prod_{i<j}^{n} S_{b}\left( \pm\left(\mathrm{i} x_{i}-\mathrm{i} x_{j}\right)\right)}
$$

for $\sum_{r=0}^{2 m+2 n+3} \nu_{r}=(m+1)\left(b+b^{-1}\right)$. Corollary 4.2 of [58] states the invariance under $m \leftrightarrow n$ and $\nu \rightarrow \frac{1}{2}\left(b+b^{-1}\right)-\nu$ :

$$
I_{B C_{n} ; h}^{(m)}\left(\nu_{0}, \ldots, \nu_{2 m+2 n+3}\right)=\left[\prod_{r<s}^{2 m+2 n+3} S_{b}\left(\nu_{r}+\nu_{s}\right)\right] I_{B C_{m} ; h}^{(n)}\left(\frac{b+b^{-1}}{2}-\nu_{0}, \frac{b+b^{-1}}{2}-\nu_{1}, \ldots\right) .
$$


The $S_{b}^{3}$ partition function (5.9) of the USp $(2 N-2)$ SQCD with $4 N$ chiral multiplets which we studied earlier is such a hyperbolic hypergeometric integral, with $m=n=N-1$ and $4 N$ parameters $\nu_{r}$ summing to $N\left(b+b^{-1}\right)$ :

$$
\begin{aligned}
Z_{S_{b}^{3}}=I_{B C_{N-1} ; h}^{(N-1)}\left(\frac{b+b^{-1}}{4}-\mathrm{i} \hat{m}_{f}-\mathrm{i} \mu(f=1, \ldots, 2 N),\right. \\
\left.\frac{b+b^{-1}}{4}-\mathrm{i} m+\mathrm{i} a_{s}+\mathrm{i} \mu, \frac{b+b^{-1}}{4}+\mathrm{i} m+\mathrm{i} a_{s}^{\prime}+\mathrm{i} \mu(s=1, \ldots, N)\right) .
\end{aligned}
$$

The identity (5.18) states that $Z_{S_{b}^{3}}$ is invariant up to a factor $\prod_{r<s} S_{b}\left(\nu_{r}+\nu_{s}\right)$ under changing the signs of all $\mu, \hat{m}_{f}, m, a_{s}, a_{s}^{\prime}$.

We now take the limit $\mu \rightarrow \pm \infty$ in (5.18), after multiplying by $E(\mu)$ and $E(-\mu)$ respectively as explained in (5.13). On each side, we obtain partition functions of $\mathrm{U}(N-1)$ theories, and the product of $S_{b}\left(\nu_{r}+\nu_{s}\right)$ reads

$$
\begin{aligned}
\prod_{r<s}^{4 N-1} S_{b}\left(\nu_{r}+\nu_{s}\right)= & \prod_{f=1}^{2 N} \prod_{s=1}^{N}\left[S_{b}\left(\frac{b+b^{-1}}{2}-\mathrm{i} \hat{m}_{f}-\mathrm{i} m+\mathrm{i} a_{s}\right) S_{b}\left(\frac{b+b^{-1}}{2}-\mathrm{i} \hat{m}_{f}+\mathrm{i} m+\mathrm{i} a_{s}^{\prime}\right)\right] \\
& \times e^{-\mathrm{i} \pi\left(\sum_{f=1}^{2 N} \hat{m}_{f}^{2}-\sum_{s=1}^{N} a_{s}^{2}-\sum_{s=1}^{N} a_{s}^{\prime 2}-2 N m^{2}\right)(N-1) \operatorname{sign} \mu}
\end{aligned}
$$

The phases are exactly $E(-\mu) / E(\mu)$ with $E$ defined below (5.13). The remaining $S_{b}$ functions are one-loop determinants of mesons formed as the product of a fundamental and an antifundamental chiral multiplet under $\mathrm{U}(N-1)$. The full $S_{b}^{4}$ partition function (5.3) of the $4 \mathrm{~d} / 3 \mathrm{~d}$ coupled system also includes one-loop determinants of hypermultiplets on halfellipsoids, which are $\Gamma_{b}$ functions with the same arguments as the $S_{b}$ functions in (5.20) up to signs. Since

$$
S_{b}\left(\frac{b+b^{-1}}{2}+x\right)=\frac{\Gamma_{b}\left(\frac{b+b^{-1}}{2}+x\right)}{\Gamma_{b}\left(\frac{b+b^{-1}}{2}-x\right)},
$$

the $S_{b}$ functions coming from mesons in the 3 d duality convert between $\Gamma_{b}\left(\frac{b+b^{-1}}{2} \pm x\right)$. This is fully consistent with $4 \mathrm{~d}$ charge conjugation. Identical calculations show that the braiding kernel is invariant under Toda CFT charge conjugation.

The identity (5.18) was proven in [58] as a hyperbolic limit of an elliptic hypergeometric integral identity. In physics terms, the elliptic identity states that two Seiberg-dual $4 \mathrm{~d}$ $\mathcal{N}=1$ theories with $\operatorname{USp}(2 N-2)$ gauge group and $4 N$ fundamental chiral multiplets have the same $S_{b}^{3} \times S^{1}$ partition function (supersymmetric index). Note that while the dual of $4 \mathrm{~d} \mathcal{N}=1 \mathrm{USp}\left(2 N_{c}\right)$ SQCD with $2 N_{f}$ fundamental chiral multiplets has $\widetilde{N}_{c}=N_{f}-N_{c}-2$ colors, the analoguous $3 \mathrm{~d} \mathcal{N}=2$ Aharony duality has $\widetilde{N}_{c}=N_{f}-N_{c}-1$ colors instead [43]. For our case $N_{c}=N-1$ and $N_{f}=2 N$ the $4 \mathrm{~d}$ theory is self-dual but the $3 \mathrm{~d}$ theory is not. On the other hand, Aharony duality can be retrieved as a limit of (5.18) when $\nu_{2 m+2 n+2},-\nu_{2 m+2 n+3} \rightarrow \mathrm{i} \infty$. This is physically interpreted as dimensional reduction of $4 \mathrm{~d}$ USp dualities down to $3 \mathrm{~d}$ following $[59,60]$.

Let us finish this section with a mention of $N=2$. Besides the USp-type Seiberg duality there is also an $S U$-type Seiberg duality in $4 \mathrm{~d}$ in that case, thanks to the accidental isomorphism $\mathrm{USp}(2)=\mathrm{SU}(2)$. The map of parameters for the $\mathrm{SU}(2)$ Seiberg duality 
depends on a split of the 8 chiral multiplets into 4 "fundamental" and 4 "antifundamental" ones. After descending to $3 \mathrm{~d} \mathcal{N}=2$ partition functions,

$$
\begin{aligned}
& I_{B C_{1} ; h}^{(1)}\left(\nu_{0}, \ldots, \nu_{7}\right) \\
& =\prod_{r=0}^{3} \prod_{s=4}^{7}\left[S_{b}\left(\nu_{r}+\nu_{s}\right)\right] I_{B C_{1} ; h}^{(1)}\left(\frac{\nu_{0}+\nu_{1}}{2} \pm \frac{\nu_{2}-\nu_{3}}{2}, \frac{\nu_{2}+\nu_{3}}{2} \pm \frac{\nu_{0}-\nu_{1}}{2}, \frac{\nu_{4}+\nu_{5}}{2} \pm \frac{\nu_{6}-\nu_{7}}{2}, \frac{\nu_{6}+\nu_{7}}{2} \pm \frac{\nu_{4}-\nu_{5}}{2}\right) .
\end{aligned}
$$

As for general $N$, we are interested in a limit where the $\nu$ go to $\pm \mathrm{i} \infty$ (half with each sign). We can in particular choose $+\nu_{0},-\nu_{1},-\nu_{2},-\nu_{3},+\nu_{4},+\nu_{5},+\nu_{6},-\nu_{7} \sim+\mathrm{i} \mu$. Then most parameters in the dual (5.22) remain finite: only the first and the last depend on $\mu$. The limit $\mu \rightarrow \infty$ then takes the $S_{b}^{3}$ partition function of SU(2) SQCD with 8 doublets to that of $\mathrm{SU}(2) \mathrm{SQCD}$ with 6 doublets. This reproduces the $3 \mathrm{~d} \mathcal{N}=2$ description found in [37] for the S-duality domain wall of $4 \mathrm{~d} \mathcal{N}=2 \mathrm{SU}(2) \mathrm{SQCD}$ with 4 flavours.

For $N=2$, our description of the domain wall as a $\mathrm{U}(N-1)=\mathrm{U}(1)$ theory, namely 3d $\mathcal{N}=2$ SQED with $N_{f}=4$ flavours and a monopole superpotential was also found to be dual to $\mathrm{SU}(2)$ with 6 doublets in [41] by realizing both from dimensional reduction of a 4 d theory with $E_{7}$ symmetry. It would be interesting to see if a similar construction could be performed for $N>2$.

\section{Conclusions}

In this work we have determined the integral kernel (4.4) which braids two semi-degenerate vertex operators of the Toda CFT. Through the AGT relation, we have deduced the ellipsoid expectation value of an S-duality domain wall in $4 \mathrm{~d} \mathcal{N}=2 \mathrm{SU}(N)$ SQCD with $2 N$ flavours. We have then described the wall by coupling $3 \mathrm{~d} \mathcal{N}=2 \mathrm{U}(N-1)$ SQCD with $2 N+2 N$ chiral multiplets and a monopole superpotential on the wall to the $4 \mathrm{~d}$ theories on both sides of the wall.

The shift relations found in section 4.3 do not appear sufficient to prove that the braiding kernel is correct. An obvious question would be to fill in this gap by checking additional Moore-Seiberg relations. It should be possible to extract Racah-Wigner coefficients for the modular double of $U_{q}\left(s l_{N}\right)$, as was done for $N=2$ in [44,45], and to get $6 \mathrm{j}$ symbols of $U_{q}\left(s l_{N}\right)$ by taking a limit of degenerate momenta, thus generalizing [61] from four symmetric to two symmetric representations of $U_{q}\left(s l_{N}\right)$.

It would be valuable to evaluate one-loop determinants of hypermultiplets and vector multiplets on the half-ellipsoid with appropriate boundary conditions, and clarify whether the result indeed consists of half of the $\Gamma_{b}$ factors in the full-ellipsoid results.

S-duality is expected to map Wilson loops to 't Hooft loops. Expectation values of Wilson loop and 't Hooft loop observables on the ellipsoid are known exactly [47,62] and we now know the explicit S-duality kernel. Conjugating the Wilson loop by this kernel should thus yield the 't Hooft loop, namely if one considers the $4 \mathrm{~d}$ theory on $S_{b}^{4}$ with two S-duality walls near the equator and a Wilson loop in between, then the collision limit should yield a 't Hooft loop. In fact, a preliminary question is to understand how the collision of two domain walls which perform S-duality and its inverse yields a trivial 
operator. This annihilation should involve appropriate 3d dualities, as happens in one dimension higher in [30].

The S-duality wall generalizes straightforwardly to the case where part (or all) of the $\mathrm{SU}(2 N)$ flavour symmetry shared by the two $4 \mathrm{~d}$ theories is gauged by $4 \mathrm{~d}$ vector multiplets. Class $\mathrm{S}$ theories, constructed by twisted dimensional reduction of the $6 \mathrm{~d}(2,0) \mathrm{SU}(N)$ superconformal theory on a punctured Riemann surface $\Sigma$, provide interesting examples, for instance linear quivers of $\mathrm{SU}(N)$ gauge groups. The $3 \mathrm{~d}$ description of an S-duality wall in such a quiver is again $3 \mathrm{~d} \mathcal{N}=2 \mathrm{U}(N-1)$ SQCD coupled with $4 \mathrm{~d}$ fields through cubic superpotentials:

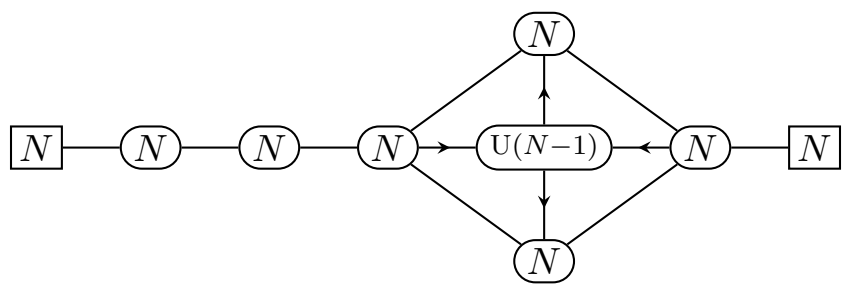

Quiver gauge theories open up the possibility of colliding S-duality domain walls, where S-duality acts on different gauge groups $\mathrm{SU}(N)$. When the groups are separated, the $3 \mathrm{~d}$ description is obvious (on the Toda side the braiding operations commute):

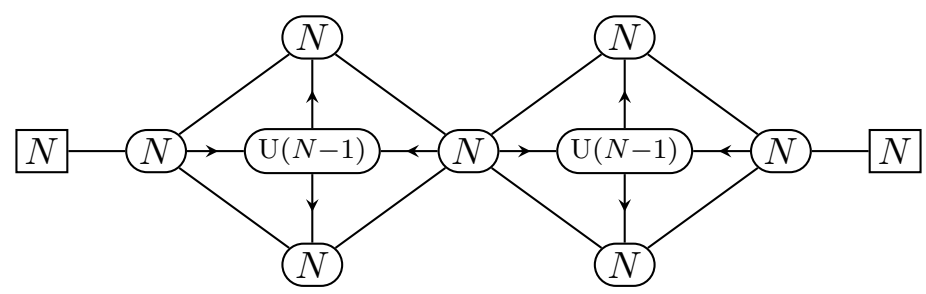

When the two groups share a bifundamental hypermultiplet, the duality walls do not commute and we propose the following descriptions for the two possible orderings:

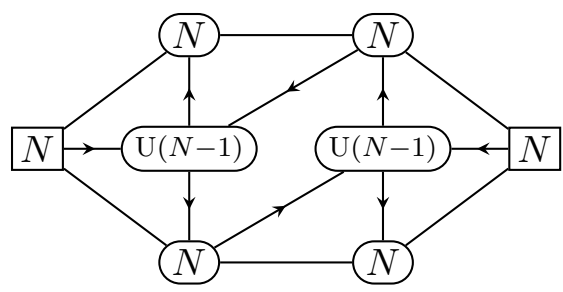

vs

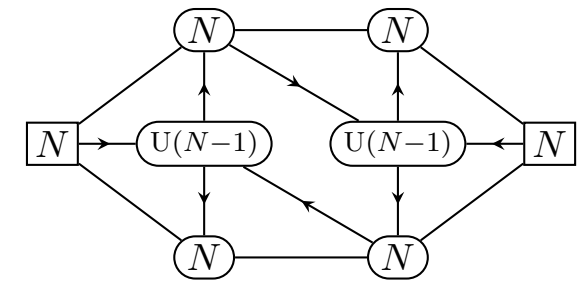

with a cubic superpotential term coupling $3 \mathrm{~d}$ and $4 \mathrm{~d}$ fields for each triangle and a quartic superpotential for 3d chiral multiplets in the central paralellogram, as well as suitable monopole superpotentials. Such combinations of S-duality walls have been investigated (after version 1 of the present paper) in [63].

From the Toda CFT point of view, each of these products of duality walls corresponds to a product of two $W_{N}$ braiding kernels. It may be interesting to translate Moore-Seiberg relations of braiding kernels into the gauge theory language and understand how $3 \mathrm{~d} \mathcal{N}=2$ dualities reproduce them.

Note that all of this work focused on gauge theories with a Lagrangian description, or equivalently Toda CFT correlators with "enough" degeneracy. In particular, we have 
avoided the limit $x \rightarrow 1$ of $4 \mathrm{~d} \mathcal{N}=2$ SQCD, which involves a strongly coupled matter theory instead of hypermultiplets, coupled to a vector multiplet. For $N=3$ this theory includes $T_{3}$. The corresponding crossing symmetry on the Toda CFT side consists of the fusion of two simple punctures into a less degenerate operator $\widehat{V}_{\alpha}$. Conformal blocks in this limit are not uniquely characterized by $\alpha$ and external operators, and one needs a label for the continuous multiplicity with which $\widehat{V}_{\alpha}$ appears in the fusion of the two full punctures. These conformal blocks are eigenfunctions of the square of the braiding kernel, and it is tempting to try and diagonalize this kernel. Unfortunately, we only succeeded to tame multiplicities in the simplest discrete versions of the kernel, and could not generalize.

Another direction worth pursuing is to consider S-duality walls in $4 \mathrm{~d} \mathcal{N}=2$ theories with gauge groups such as $\operatorname{Sp}(N)$ (see for instance [64]). Too little is known at present about braiding kernels of D-type and E-type Toda CFT in order to apply the techniques used here. Understanding whether the $\mathrm{U}(N-1) 3 \mathrm{~d}$ theory found in this paper can be derived through brane constructions may help in generalizing to other gauge groups by orbifolding.

It would also be interesting to check that our gauge theory description of the S-duality wall gives the correct $S_{b}^{3} \times S^{1}$ index, as was done for $\mathrm{SU}(2)$ theories in [65-68].

\section{Acknowledgments}

I thank Francesco Benini, Bertrand Eynard, Yale Fan, Davide Gaiotto, Igor Klebanov, Márk Mezei, Satoshi Nawata, Sara Pasquetti, Silviu Pufu, Shlomo Razamat, Sylvain Ribault, Nathan Seiberg, Jan Troost, Ran Yacoby and especially Jaume Gomis for discussions, comments, and pointers to the literature.

Open Access. This article is distributed under the terms of the Creative Commons Attribution License (CC-BY 4.0), which permits any use, distribution and reproduction in any medium, provided the original author(s) and source are credited.

\section{References}

[1] K.G. Wilson, Confinement of Quarks, Phys. Rev. D 10 (1974) 2445 [InSPIRE].

[2] G. 't Hooft, On the Phase Transition Towards Permanent Quark Confinement, Nucl. Phys. B 138 (1978) 1 [INSPIRE].

[3] S. Gukov and E. Witten, Gauge Theory, Ramification, And The Geometric Langlands Program, hep-th/0612073 [INSPIRE].

[4] S. Gukov and E. Witten, Rigid Surface Operators, Adv. Theor. Math. Phys. 14 (2010) 87 [arXiv: 0804.1561] [INSPIRE].

[5] S. Gukov, Surface Operators, in New Dualities of Supersymmetric Gauge Theories, J. Teschner ed., pp. 223-259 (2016) [DOI] [arXiv:1412.7127] [INSPIRE].

[6] D. Gaiotto and E. Witten, Supersymmetric Boundary Conditions in $\mathcal{N}=4$ Super Yang-Mills Theory, J. Statist. Phys. 135 (2009) 789 [arXiv:0804.2902] [INSPIRE].

[7] D. Gaiotto and E. Witten, Janus Configurations, Chern-Simons Couplings, And The theta-Angle in $\mathcal{N}=4$ Super Yang-Mills Theory, JHEP 06 (2010) 097 [arXiv:0804.2907] [INSPIRE]. 
[8] D. Gaiotto and E. Witten, S-duality of Boundary Conditions In $\mathcal{N}=4$ Super Yang-Mills Theory, Adv. Theor. Math. Phys. 13 (2009) 721 [arXiv:0807.3720] [InSPIRE].

[9] S. Gukov and A. Kapustin, Topological Quantum Field Theory, Nonlocal Operators, and Gapped Phases of Gauge Theories, arXiv:1307.4793 [INSPIRE].

[10] N. Seiberg and E. Witten, Electric-magnetic duality, monopole condensation, and confinement in $\mathcal{N}=2$ supersymmetric Yang-Mills theory, Nucl. Phys. B 426 (1994) 19 [Erratum ibid. 430 (1994) 485] [hep-th/9407087] [INSPIRE].

[11] D. Gaiotto, $\mathcal{N}=2$ dualities, JHEP 08 (2012) 034 [arXiv:0904.2715] [INSPIRE].

[12] A. Kapustin, Wilson-'t Hooft operators in four-dimensional gauge theories and S-duality, Phys. Rev. D 74 (2006) 025005 [hep-th/0501015] [INSPIRE].

[13] L.F. Alday, D. Gaiotto, S. Gukov, Y. Tachikawa and H. Verlinde, Loop and surface operators in $\mathcal{N}=2$ gauge theory and Liouville modular geometry, JHEP 01 (2010) 113 [arXiv: 0909.0945] [INSPIRE].

[14] N. Drukker, J. Gomis, T. Okuda and J. Teschner, Gauge Theory Loop Operators and Liouville Theory, JHEP 02 (2010) 057 [arXiv:0909.1105] [INSPIRE].

[15] N. Drukker, D. Gaiotto and J. Gomis, The Virtue of Defects in $4 D$ Gauge Theories and 2D CFTs, JHEP 06 (2011) 025 [arXiv: 1003.1112] [INSPIRE].

[16] J. Fuchs, C. Schweigert and K. Waldorf, Bi-branes: Target space geometry for world sheet topological defects, J. Geom. Phys. 58 (2008) 576 [hep-th/0703145] [INSPIRE].

[17] J. Fuchs, M.R. Gaberdiel, I. Runkel and C. Schweigert, Topological defects for the free boson CFT, J. Phys. A 40 (2007) 11403 [arXiv:0705.3129] [inSPIRE].

[18] G. Sarkissian and C. Schweigert, Some remarks on defects and T-duality, Nucl. Phys. B $\mathbf{8 1 9}$ (2009) 478 [arXiv: 0810.3159] [INSPIRE].

[19] A. Kapustin and M. Tikhonov, Abelian duality, walls and boundary conditions in diverse dimensions, JHEP 11 (2009) 006 [arXiv:0904.0840] [INSPIRE].

[20] M.-C. Tan, Surface Operators in Abelian Gauge Theory, JHEP 05 (2009) 104 [arXiv: 0904.1744] [INSPIRE].

[21] M.-C. Tan, Nonlocal Operators and Duality in Abelian Gauge Theory on a Four-Manifold, arXiv:1312.5494 [INSPIRE].

[22] L. Martucci, Topological duality twist and brane instantons in F-theory, JHEP 06 (2014) 180 [arXiv: 1403.2530] [INSPIRE].

[23] K. Hosomichi, S. Lee and J. Park, AGT on the S-duality Wall, JHEP 12 (2010) 079 [arXiv: 1009.0340] [INSPIRE].

[24] Y. Terashima and M. Yamazaki, SL $(2, \mathbb{R})$ Chern-Simons, Liouville, and Gauge Theory on Duality Walls, JHEP 08 (2011) 135 [arXiv:1103.5748] [INSPIRE].

[25] Y. Terashima and M. Yamazaki, Semiclassical Analysis of the 3d/3d Relation, Phys. Rev. D 88 (2013) 026011 [arXiv:1106.3066] [INSPIRE].

[26] T. Dimofte, D. Gaiotto and S. Gukov, Gauge Theories Labelled by Three-Manifolds, Commun. Math. Phys. 325 (2014) 367 [arXiv:1108.4389] [InSPIRE].

[27] A. Gadde, S. Gukov and P. Putrov, Walls, Lines, and Spectral Dualities in 3d Gauge Theories, JHEP 05 (2014) 047 [arXiv:1302.0015] [INSPIRE].

[28] T. Dimofte, D. Gaiotto and R. van der Veen, $R G$ Domain Walls and Hybrid Triangulations, Adv. Theor. Math. Phys. 19 (2015) 137 [arXiv:1304.6721] [INSPIRE]. 
[29] D. Gaiotto and S.S. Razamat, $\mathcal{N}=1$ theories of class $\mathcal{S}_{k}$, JHEP 07 (2015) 073 [arXiv: 1503.05159] [INSPIRE].

[30] D. Gaiotto and H.-C. Kim, Duality walls and defects in $5 d \mathcal{N}=1$ theories, JHEP 01 (2017) 019 [arXiv: 1506.03871] [INSPIRE].

[31] A. Hashimoto, P. Ouyang and M. Yamazaki, Boundaries and defects of $\mathcal{N}=4$ SYM with 4 supercharges. Part I: Boundary/junction conditions, JHEP 10 (2014) 107 [arXiv: 1404.5527] [INSPIRE].

[32] A. Hashimoto, P. Ouyang and M. Yamazaki, Boundaries and defects of $\mathcal{N}=4$ SYM with 4 supercharges. Part II: Brane constructions and $3 d \mathcal{N}=2$ field theories, JHEP 10 (2014) 108 [arXiv:1406.5501] [INSPIRE].

[33] L.F. Alday, D. Gaiotto and Y. Tachikawa, Liouville Correlation Functions from Four-dimensional Gauge Theories, Lett. Math. Phys. 91 (2010) 167 [arXiv:0906.3219] [INSPIRE].

[34] N. Wyllard, $A_{N-1}$ conformal Toda field theory correlation functions from conformal $\mathcal{N}=2$ $\mathrm{SU}(N)$ quiver gauge theories, JHEP 11 (2009) 002 [arXiv:0907.2189] [INSPIRE].

[35] N. Hama and K. Hosomichi, Seiberg-Witten Theories on Ellipsoids, JHEP 09 (2012) 033 [Addendum ibid. 10 (2012) 051] [arXiv:1206.6359] [INSPIRE].

[36] T. Nosaka and S. Terashima, Supersymmetric Gauge Theories on a Squashed Four-Sphere, JHEP 12 (2013) 001 [arXiv:1310.5939] [InSPIRE].

[37] J. Teschner and G. Vartanov, 6j symbols for the modular double, quantum hyperbolic geometry, and supersymmetric gauge theories, Lett. Math. Phys. 104 (2014) 527 [arXiv: 1202.4698] [INSPIRE].

[38] D.L. Jafferis, The Exact Superconformal R-Symmetry Extremizes Z, JHEP 05 (2012) 159 [arXiv: 1012.3210] [INSPIRE].

[39] N. Hama, K. Hosomichi and S. Lee, Notes on SUSY Gauge Theories on Three-Sphere, JHEP 03 (2011) 127 [arXiv: 1012.3512] [INSPIRE].

[40] N. Hama, K. Hosomichi and S. Lee, SUSY Gauge Theories on Squashed Three-Spheres, JHEP 05 (2011) 014 [arXiv: 1102.4716] [INSPIRE].

[41] T. Dimofte and D. Gaiotto, An E7 Surprise, JHEP 10 (2012) 129 [arXiv:1209.1404] [INSPIRE].

[42] F. Benini, S. Benvenuti and S. Pasquetti, SUSY monopole potentials in $2+1$ dimensions, JHEP 08 (2017) 086 [arXiv:1703.08460] [INSPIRE].

[43] O. Aharony, IR duality in $d=3 N=2$ supersymmetric $\operatorname{USp}\left(2 N_{c}\right)$ and $\mathrm{U}\left(N_{c}\right)$ gauge theories, Phys. Lett. B 404 (1997) 71 [hep-th/9703215] [INSPIRE].

[44] B. Ponsot and J. Teschner, Liouville bootstrap via harmonic analysis on a noncompact quantum group, hep-th/9911110 [INSPIRE].

[45] B. Ponsot and J. Teschner, Clebsch-Gordan and Racah-Wigner coefficients for a continuous series of representations of $U_{q}(\mathrm{SL}(2, \mathbb{R}))$, Commun. Math. Phys. 224 (2001) 613 [math/0007097] [iNSPIRE].

[46] G.W. Moore and N. Seiberg, Polynomial Equations for Rational Conformal Field Theories, Phys. Lett. B 212 (1988) 451 [INSPIRE].

[47] V. Pestun, Localization of gauge theory on a four-sphere and supersymmetric Wilson loops, Commun. Math. Phys. 313 (2012) 71 [arXiv:0712.2824] [InSPIRE]. 
[48] S. Sugishita and S. Terashima, Exact Results in Supersymmetric Field Theories on Manifolds with Boundaries, JHEP 11 (2013) 021 [arXiv: 1308.1973] [INSPIRE].

[49] D. Honda and T. Okuda, Exact results for boundaries and domain walls in $2 d$ supersymmetric theories, JHEP 09 (2015) 140 [arXiv:1308.2217] [INSPIRE].

[50] K. Hori and M. Romo, Exact Results In Two-Dimensional $(2,2)$ Supersymmetric Gauge Theories With Boundary, arXiv:1308.2438 [INSPIRE].

[51] M. Bullimore, Defect Networks and Supersymmetric Loop Operators, JHEP 02 (2015) 066 [arXiv: 1312.5001] [INSPIRE].

[52] P. Gavrylenko, N. Iorgov and O. Lisovyy, Higher rank isomonodromic deformations and $W$-algebras, Lett. Math. Phys. 110 (2019) 327 [arXiv:1801.09608] [INSPIRE].

[53] V.A. Fateev and A.V. Litvinov, Correlation functions in conformal Toda field theory. I., JHEP 11 (2007) 002 [arXiv:0709.3806] [INSPIRE].

[54] J. Gomis and B. Le Floch, M2-brane surface operators and gauge theory dualities in Toda, JHEP 04 (2016) 183 [arXiv:1407.1852] [INSPIRE].

[55] N.E. Nørlund, Hypergeometric functions, Acta Math. 94 (1955) 289.

[56] J. Gomis and B. Le Floch, 't Hooft Operators in Gauge Theory from Toda CFT, JHEP 11 (2011) 114 [arXiv:1008.4139] [INSPIRE].

[57] M. Spreafico, On the Barnes double zeta and Gamma functions, J. Number Theory 129 (2009) 2035.

[58] E.M. Rains, Limits of elliptic hypergeometric integrals, Ramanujan J. 18 (2007) 257 [math/0607093] [INSPIRE].

[59] O. Aharony, S.S. Razamat, N. Seiberg and B. Willett, $3 d$ dualities from $4 d$ dualities, JHEP 07 (2013) 149 [arXiv: 1305.3924] [inSPIRE].

[60] O. Aharony, S.S. Razamat, N. Seiberg and B. Willett, $3 d$ dualities from $4 d$ dualities for orthogonal groups, JHEP 08 (2013) 099 [arXiv: 1307.0511] [INSPIRE].

[61] S. Nawata, P. Ramadevi and Zodinmawia, Multiplicity-free quantum $6 j$-symbols for $U_{q}\left(\mathfrak{s l}_{N}\right)$, Lett. Math. Phys. 103 (2013) 1389 [arXiv:1302.5143] [INSPIRE].

[62] J. Gomis, T. Okuda and V. Pestun, Exact Results for 't Hooft Loops in Gauge Theories on $S^{4}$, JHEP 05 (2012) 141 [arXiv:1105.2568] [INSPIRE].

[63] I. Garozzo, N. Mekareeya and M. Sacchi, Duality walls in the $4 d \mathcal{N}=2 \mathrm{SU}(N)$ gauge theory with $2 N$ flavours, JHEP 11 (2019) 053 [arXiv:1909.02832] [INSPIRE].

[64] K.-M. Lee and P. Yi, A Family of $N=2$ gauge theories with exact $S$ duality, Nucl. Phys. $B$ 520 (1998) 157 [hep-th/9706023] [INSPIRE].

[65] D. Gang, E. Koh and K. Lee, Superconformal Index with Duality Domain Wall, JHEP 10 (2012) 187 [arXiv:1205.0069] [INSPIRE].

[66] E. Koh, Duality domain wall index on $S^{1} \times S^{3}$, Int. J. Mod. Phys. Conf. Ser. 21 (2013) 182 [INSPIRE].

[67] D. Gang, E. Koh, S. Lee and J. Park, Superconformal Index and 3d-3d Correspondence for Mapping Cylinder/Torus, JHEP 01 (2014) 063 [arXiv:1305.0937] [INSPIRE].

[68] M. Bullimore, M. Fluder, L. Hollands and P. Richmond, The superconformal index and an elliptic algebra of surface defects, JHEP 10 (2014) 062 [arXiv:1401.3379] [INSPIRE]. 